TRANSACTIONS OF THE

AMERICAN MATHEMATICAL SOCIETY

Volume 364, Number 12, December 2012, Pages 6531-6564

S 0002-9947(2012)05597-8

Article electronically published on May 29, 2012

\title{
TENSOR PRODUCT MULTIPLICITIES FOR CRYSTAL BASES OF EXTREMAL WEIGHT MODULES OVER QUANTUM INFINITE RANK AFFINE ALGEBRAS OF TYPES $B_{\infty}, C_{\infty}$, AND $D_{\infty}$
}

\author{
SATOSHI NAITO AND DAISUKE SAGAKI
}

\begin{abstract}
Using Lakshmibai-Seshadri paths, we give a combinatorial realization of the crystal basis of an extremal weight module of a (general) integral extremal weight over the quantized universal enveloping algebra associated to the infinite rank affine Lie algebra of type $B_{\infty}, C_{\infty}$, or $D_{\infty}$. Moreover, via this realization, we obtain an explicit description (in terms of LittlewoodRichardson coefficients) of how tensor products of these crystal bases decompose into connected components when their extremal weights are of nonnegative levels. These results in types $B_{\infty}, C_{\infty}$, and $D_{\infty}$ extend the corresponding results due to Kwon in types $A_{+\infty}$ and $A_{\infty}$. Our results above also include, as a special case, the corresponding results (concerning crystal bases) due to Lecouvey in types $B_{\infty}, C_{\infty}$, and $D_{\infty}$, where the extremal weights are of level zero.
\end{abstract}

\section{INTRODUCTION}

Let $U_{q}(\mathfrak{g})$ be the quantized universal enveloping algebra over $\mathbb{C}(q)$ associated to the infinite rank affine Lie algebra $\mathfrak{g}$ of type $A_{+\infty}, A_{\infty}, B_{\infty}, C_{\infty}$, or $D_{\infty}$ with Cartan subalgebra $\mathfrak{h}=\bigoplus_{i \in I} \mathbb{C} h_{i}$ and integral weight lattice $P=\bigoplus_{i \in I} \mathbb{Z} \Lambda_{i} \subset \mathfrak{h}^{*}$, where $I$ is the (infinite) index set for the simple roots. In Kw2, Kw3, Kwon studied the crystal basis $\mathcal{B}(\lambda)$ of the extremal weight $U_{q}(\mathfrak{g})$-module $V(\lambda)$ of extremal weight $\lambda \in P$ in the cases where $\mathfrak{g}$ is of type $A_{+\infty}$ and type $A_{\infty}$. In these papers, he gave a combinatorial realization of the crystal basis $\mathcal{B}(\lambda)$ for $\lambda \in P$ of level zero (see also [Kw1, §4.1] for the case of dominant $\lambda \in P$ ) by using semistandard Young tableaux in which the entries are the crystal basis elements of the vector representation of $U_{q}(\mathfrak{g})$. Furthermore, he explicitly described (in terms of Littlewood-Richardson coefficients) how the tensor product $\mathcal{B}(\lambda) \otimes \mathcal{B}(\mu)$ decomposes into connected components when $\lambda, \mu \in P$ are of nonnegative levels. Also, in [Le, Lecouvey studied the crystal bases (and canonical bases) of certain irreducible $U_{q}(\mathfrak{g})$-modules, which are neither highest weight modules nor lowest weight modules, in the cases where $\mathfrak{g}$ is of type $A_{+\infty}, B_{\infty}, C_{\infty}$, and $D_{\infty}$, and furthermore

Received by the editors May 11, 2010 and, in revised form, December 29, 2010 and April 1, 2011.

2010 Mathematics Subject Classification. Primary 17B37; Secondary 05E10, 05A19, 17 B67.

(C)2012 American Mathematical Society
Reverts to public domain 28 years from publication 
showed that tensor product multiplicities for these modules are precisely the corresponding Littlewood-Richardson coefficients (not depending on the types $A_{+\infty}$, $B_{\infty}, C_{\infty}$, and $\left.D_{\infty}\right)$. These modules will surely turn out to be extremal weight $U_{q}(\mathfrak{g})$-modules of extremal weight of level zero.

In this paper, we extend the results of [Kw2, Kw3] (in types $A_{+\infty}$ and $A_{\infty}$ ) above to the cases of types $B_{\infty}, C_{\infty}$, and $D_{\infty}$ in such a way that the results (concerning crystal bases) of [Le] above are also included, though we make use of results in Le (see 44.1 ). We emphasize that our approach is quite different from those in Kw2], Kw3, and Le. In fact, we prove our results entirely within the framework of Littelmann's path model, which enables us to give a unified proof in all types $B_{\infty}, C_{\infty}$, and $D_{\infty}$, though for simplicity of notation, we give a proof of Proposition 4.10 only for the case of type $C_{\infty}$ (see \$5). However, we should mention that the description in terms of Kashiwara-Nakashima tableaux of the crystal basis $\mathcal{B}(\lambda)$ for an arbitrary $\lambda \in P$ of nonnegative level would probably be more efficient for explicit computations (if one obtains it).

Let us explain our results more precisely. We set $I:=\mathbb{Z}_{\geq 0}$, and $[m]:=\{0,1, \ldots$, $m\}$ for $m \in \mathbb{Z}_{\geq 0}$. Also, we denote by $P_{+} \subset P$ the set of dominant integral weights, and by $E$ the subset of $P$ consisting of all elements of level zero. For $\lambda \in P$, let $\mathbb{B}(\lambda)$ denote the $U_{q}(\mathfrak{g})$-crystal consisting of all Lakshmibai-Seshadri (LS for short) paths of shape $\lambda$. Our first main results (Theorem 3.20 and Corollary 3.21) state that for each $\lambda \in P$, the $U_{q}(\mathfrak{g})$-crystal $\mathbb{B}(\lambda)$ provides a combinatorial realization of the crystal basis $\mathcal{B}(\lambda)$. In addition, we see that the crystal graph of $\mathcal{B}(\lambda) \cong \mathbb{B}(\lambda)$ is connected. Then, by means of this realization of $\mathcal{B}(\lambda)$, we study how the tensor product $\mathcal{B}(\lambda) \otimes \mathcal{B}(\mu) \cong \mathbb{B}(\lambda) \otimes \mathbb{B}(\mu)$ decomposes into connected components when $\lambda, \mu \in P$ are of nonnegative levels, dividing the problem into four cases: the case $\lambda, \mu \in E$ (see 4.1); the case $\lambda \in E, \mu \in P_{+}$(see 4.2 ); the case $\lambda \in P_{+}, \mu \in E$ (see 4 4.3); the case $\lambda, \mu \in P_{+}$(see $\$ 4.4$ ). It turns out that in all the cases above, each connected component of the tensor product $\mathbb{B}(\lambda) \otimes \mathbb{B}(\mu)$ is isomorphic to $\mathbb{B}(\nu)(\cong \mathcal{B}(\nu))$ for some $\nu \in P$ of nonnegative level. In particular, in the (most difficult) case $\lambda \in P_{+}, \mu \in E$, our result (Theorem 4.13) states that for some $m \in \mathbb{Z}_{\geq 3}$, the multiplicity of each connected component $\mathbb{B}(\nu)$ in $\mathbb{B}(\lambda) \otimes \mathbb{B}(\mu)$ is equal to the (corresponding) tensor product multiplicity of finite-dimensional irreducible highest weight $U_{q}\left(\mathfrak{g}_{[m]}\right)$-modules of highest weight $\lambda, \mu$, and $\nu$, respectively. Here, $\mathfrak{g}_{[m]}$ denotes the "reductive" Lie subalgebra of $\mathfrak{g}$ (of type $B_{m+1}, C_{m+1}$, or $D_{m+1}$ ) corresponding to the subset $[m] \subset I=\mathbb{Z}_{\geq 0}$. Note that this $m \in \mathbb{Z}_{\geq 3}$ does not depend on the connected components $\mathbb{B}(\nu)$. Moreover, by virtue of tensor product multiplicity formulas in [Ko1] and [Ko2, we obtain an explicit description of this multiplicity in terms of Littlewood-Richardson coefficients (see $\$ 5.2$ ).

Now, from the argument in 44.2 , we also observe that for each $\lambda \in P$ of nonnegative level, there exist $\lambda^{0} \in E$ and $\lambda^{+} \in P_{+}$such that $\mathbb{B}(\lambda) \cong \mathbb{B}\left(\lambda^{0}\right) \otimes \mathbb{B}\left(\lambda^{+}\right)$ as $U_{q}(\mathfrak{g})$-crystals. Therefore, by combining the results in the four cases above, we finally obtain our second main result (Theorem 4.17), which yields an explicit description (in terms of Littlewood-Richardson coefficients) of the multiplicity of each connected component $\mathbb{B}(\nu)$ in $\mathbb{B}(\lambda) \otimes \mathbb{B}(\mu)$ for general $\lambda, \mu \in P$ of nonnegative levels. 
This paper is organized as follows. In $\$ 2$, we introduce basic notation for infinite rank affine Lie algebras and their quantized universal enveloping algebras. In $₫ 3$, we first recall standard facts about crystal bases of extremal weight modules and show the connectedness of (the crystal graph of) the crystal basis $\mathcal{B}(\lambda)$ for $\lambda \in P$. Then, we show that the crystal $\mathbb{B}(\lambda)$ of all LS paths of shape $\lambda$ provides a combinatorial realization of the crystal basis $\mathcal{B}(\lambda)$ for $\lambda \in P$. In $\$ 4$, we explicitly describe how the tensor product $\mathbb{B}(\lambda) \otimes \mathbb{B}(\mu) \cong \mathcal{B}(\lambda) \otimes \mathcal{B}(\mu)$ decomposes into connected components when $\lambda, \mu \in P$ are of nonnegative levels, deferring the proof of Proposition 4.10 (used to prove Theorem 4.13) to \$5. Finally, in \$5, after reviewing tensor product multiplicity formulas in [Ko1] and [Ko2, we show Proposition 4.10, thereby completing the proof of Theorem 4.13 (and hence Theorem 4.17).

After having finished writing this article, we were informed by Jae-Hoon Kwon that in $\mathrm{Kw} 4$ he obtained a description of how the tensor product $\mathcal{B}(\lambda) \otimes \mathcal{B}(\mu)$ decomposes into connected components for $\lambda \in P_{+}$and $\mu \in-P_{+}$in the case of type $A_{\infty}$.

\section{Basic notation for infinite RANK AFFine Lie ALGEBRAs}

2.1. Infinite rank affine Lie algebras. Let $\mathfrak{g}$ be the infinite rank affine Lie algebra of type $B_{\infty}, C_{\infty}$, or $D_{\infty}$ (see [Kac, $\left.\S 7.11\right]$ ), that is, the (symmetrizable) KacMoody algebra of infinite rank associated to one of the following Dynkin diagrams:

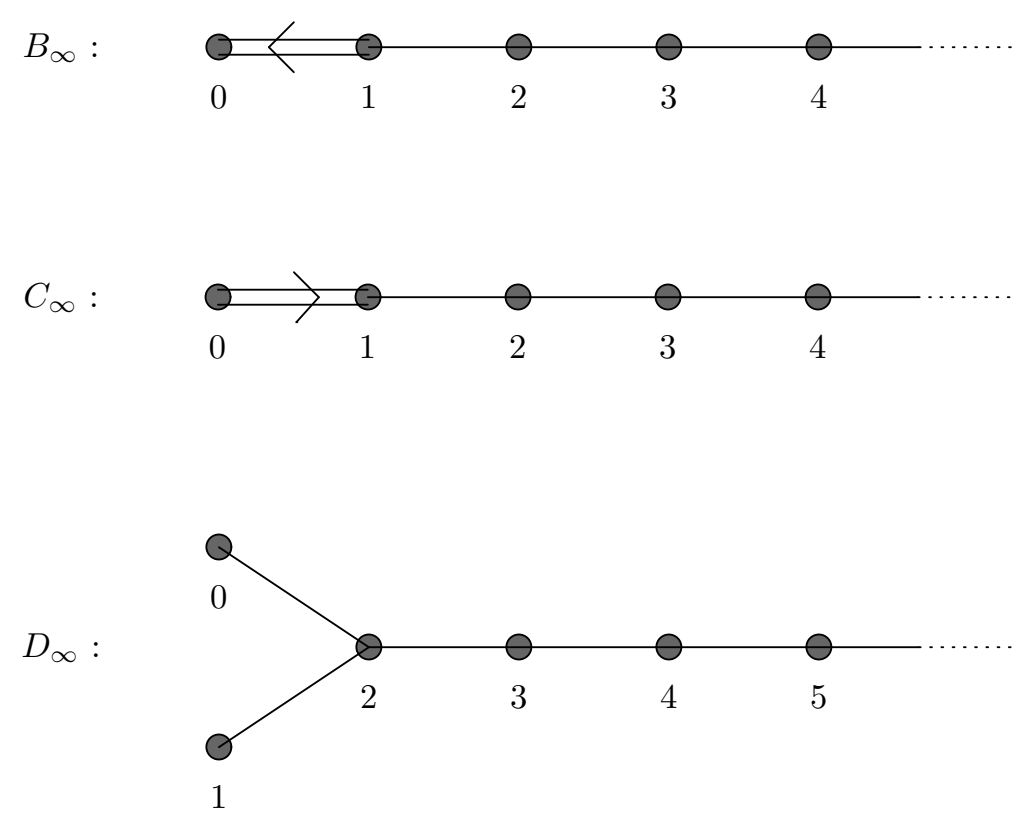

Following [Kac, $\S 7.11]$, we realize this Lie algebra $\mathfrak{g}$ as a Lie subalgebra of the Lie algebra $\mathfrak{g l}_{\infty}(\mathbb{C})$ of complex matrices $\left(a_{i j}\right)_{i, j \in \mathbb{Z}}$ with finitely many nonzero entries as follows. If $\mathfrak{g}$ is of type $B_{\infty}$ (resp., $\left.C_{\infty}, D_{\infty}\right)$, then we define elements $x_{i}, y_{i}, h_{i} \in$ 
$\mathfrak{g l}_{\infty}(\mathbb{C})$ for $i \in I:=\mathbb{Z}_{\geq 0}$ by the following equation (2.1) (resp., (2.2), (2.3)):

$$
\begin{gathered}
\left\{\begin{array}{l}
x_{0}:=E_{0,1}+E_{-1,0}, \quad x_{i}:=E_{i, i+1}+E_{-i-1,-i} \quad \text { for } i \in \mathbb{Z}_{\geq 1}, \\
y_{0}:=2\left(E_{1,0}+E_{0,-1}\right), \quad y_{i}:=E_{i+1, i}+E_{-i,-i-1} \quad \text { for } i \in \mathbb{Z}_{\geq 1}, \\
h_{0}:=2 \mathbf{e}_{0}^{\prime}, \quad h_{i}:=-\mathbf{e}_{i-1}^{\prime}+\mathbf{e}_{i}^{\prime} \quad \text { for } i \in \mathbb{Z}_{\geq 1},
\end{array}\right. \\
\left\{\begin{array}{l}
x_{0}:=E_{0,1}, \quad x_{i}:=E_{i, i+1}+E_{-i,-i+1} \quad \text { for } i \in \mathbb{Z}_{\geq 1}, \\
y_{0}:=E_{1,0}, \quad y_{i}:=E_{i+1, i}+E_{-i+1,-i} \quad \text { for } i \in \mathbb{Z}_{\geq 1}, \\
h_{0}:=\mathbf{e}_{0}^{\prime}, \quad h_{i}:=-\mathbf{e}_{i-1}^{\prime}+\mathbf{e}_{i}^{\prime} \quad \text { for } i \in \mathbb{Z}_{\geq 1},
\end{array}\right. \\
\left\{\begin{array}{l}
x_{0}:=E_{0,2}-E_{-1,1}, \quad x_{i}:=E_{i, i+1}-E_{-i,-i+1} \text { for } i \in \mathbb{Z}_{\geq 1}, \\
y_{0}:=E_{2,0}-E_{1,-1}, \quad y_{i}:=E_{i, i+1}-E_{-i+1,-i} \text { for } i \in \mathbb{Z}_{\geq 1}, \\
h_{0}:=\mathbf{e}_{0}^{\prime}+\mathbf{e}_{1}^{\prime}, \quad h_{i}:=-\mathbf{e}_{i-1}^{\prime}+\mathbf{e}_{i}^{\prime} \quad \text { for } i \in \mathbb{Z}_{\geq 1} .
\end{array}\right.
\end{gathered}
$$

Here, for each $i, j \in \mathbb{Z}, E_{i, j} \in \mathfrak{g l}_{\infty}(\mathbb{C})$ is the matrix with a 1 in the $(i, j)$ position and 0 elsewhere, and

$$
\mathbf{e}_{j}^{\prime}:=\left\{\begin{array}{ll}
E_{-j-1,-j-1}-E_{j+1, j+1} & \text { if } \mathfrak{g} \text { is of type } B_{\infty}, \\
E_{-j,-j}-E_{j+1, j+1} & \text { if } \mathfrak{g} \text { is of type } C_{\infty} \text { or } D_{\infty},
\end{array} \quad \text { for } j \in \mathbb{Z}_{\geq 0}\right.
$$

The infinite rank affine Lie algebra $\mathfrak{g}$ is isomorphic to the Lie subalgebra of $\mathfrak{g l}_{\infty}(\mathbb{C})$ generated by $x_{i}, y_{i}, h_{i}$ for $i \in I$. The elements $x_{i}, y_{i}$ for $i \in I$ are the Chevalley generators, $\mathfrak{h}:=\bigoplus_{i \in I} \mathbb{C} h_{i}$ is the Cartan subalgebra, and $\Pi^{\vee}:=\left\{h_{i}\right\}_{i \in I}$ is the set of simple coroots. Let $\Pi:=\left\{\alpha_{i}\right\}_{i \in I} \subset \mathfrak{h}^{*}:=\operatorname{Hom}_{\mathbb{C}}(\mathfrak{h}, \mathbb{C})$ be the set of simple roots for $\mathfrak{g}$. Then we have

$$
\begin{cases}\alpha_{0}=\mathbf{e}_{0}, \quad \alpha_{i}=-\mathbf{e}_{i-1}+\mathbf{e}_{i} \quad \text { for } i \in \mathbb{Z}_{\geq 1} & \text { if } \mathfrak{g} \text { is of type } B_{\infty} \\ \alpha_{0}=2 \mathbf{e}_{0}, \quad \alpha_{i}=-\mathbf{e}_{i-1}+\mathbf{e}_{i} \quad \text { for } i \in \mathbb{Z}_{\geq 1} & \text { if } \mathfrak{g} \text { is of type } C_{\infty} \\ \alpha_{0}=\mathbf{e}_{0}+\mathbf{e}_{1}, \quad \alpha_{i}=-\mathbf{e}_{i-1}+\mathbf{e}_{i} \quad \text { for } i \in \mathbb{Z}_{\geq 1} & \text { if } \mathfrak{g} \text { is of type } D_{\infty}\end{cases}
$$

Here, for each $j \in \mathbb{Z}_{\geq 0}$, we define $\mathbf{e}_{j} \in \mathfrak{h}^{*}=\operatorname{Hom}_{\mathbb{C}}(\mathfrak{h}, \mathbb{C})$ by $\left\langle\mathbf{e}_{j}, \mathbf{e}_{k}^{\prime}\right\rangle=\delta_{j k}$ for $k \in \mathbb{Z}_{\geq 0}$, where $\langle\cdot, \cdot\rangle$ denotes the natural pairing of $\mathfrak{h}^{*}$ and $\mathfrak{h}$. Note that $\mathfrak{h}=$ $\bigoplus_{j \in \mathbb{Z}_{\geq 0}} \mathbb{C e}_{j}^{\prime}$.

Let $W=\left\langle r_{i} \mid i \in I\right\rangle \subset G L\left(\mathfrak{h}^{*}\right)$ denote the Weyl group of $\mathfrak{g}$, where $r_{i}$ denotes the simple reflection corresponding to the simple root $\alpha_{i}$ for $i \in I$. If $\mathfrak{g}$ is of type $B_{\infty}$ (resp., $\left.C_{\infty}, D_{\infty}\right)$, then the following equation (2.5) (resp., (2.6), (2.7)) holds 
for $j \in \mathbb{Z}_{\geq 0}$ :

$$
r_{0}\left(\mathbf{e}_{j}\right)=\left\{\begin{array}{ll}
-\mathbf{e}_{0} & \text { if } j=0, \\
\mathbf{e}_{j} & \text { otherwise, }
\end{array} \quad r_{i}\left(\mathbf{e}_{j}\right)=\left\{\begin{array}{ll}
\mathbf{e}_{i} & \text { if } j=i-1, \\
\mathbf{e}_{i-1} & \text { if } j=i, \\
\mathbf{e}_{j} & \text { otherwise },
\end{array} \quad \text { for } i \in \mathbb{Z}_{\geq 1},\right.\right.
$$

$$
r_{0}\left(\mathbf{e}_{j}\right)=\left\{\begin{array}{ll}
-\mathbf{e}_{0} & \text { if } j=0, \\
\mathbf{e}_{j} & \text { otherwise, }
\end{array} \quad r_{i}\left(\mathbf{e}_{j}\right)=\left\{\begin{array}{ll}
\mathbf{e}_{i} & \text { if } j=i-1, \\
\mathbf{e}_{i-1} & \text { if } j=i, \\
\mathbf{e}_{j} & \text { otherwise },
\end{array} \quad \text { for } i \in \mathbb{Z}_{\geq 1},\right.\right.
$$

$$
r_{0}\left(\mathbf{e}_{j}\right)=\left\{\begin{array}{ll}
-\mathbf{e}_{1} & \text { if } j=0, \\
-\mathbf{e}_{0} & \text { if } j=1, \\
\mathbf{e}_{j} & \text { otherwise, }
\end{array} \quad r_{i}\left(\mathbf{e}_{j}\right)=\left\{\begin{array}{ll}
\mathbf{e}_{i} & \text { if } j=i-1, \\
\mathbf{e}_{i-1} & \text { if } j=i, \\
\mathbf{e}_{j} & \text { otherwise, }
\end{array} \quad \text { for } i \in \mathbb{Z}_{\geq 1} .\right.\right.
$$

Also, for the action of $W$ on $\mathfrak{h}$, entirely similar formulas hold in all the cases above, with e replaced by $\mathbf{e}^{\prime}$.

Let $\Delta:=W \Pi$ be the set of roots for $\mathfrak{g}$ and let $\Delta^{+}:=\Delta \cap \sum_{i \in I} \mathbb{Z}_{\geq 0} \alpha_{i}$ be the set of positive roots for $\mathfrak{g}$. If $\mathfrak{g}$ is of type $B_{\infty}$ (resp., $\left.C_{\infty}, D_{\infty}\right)$, then the sets $\Delta$ and $\Delta^{+}$are given by the following equation (2.8) (resp., (2.9), (2.10) ):

$$
\begin{gathered}
\left\{\begin{array}{l}
\Delta=\left\{ \pm \mathbf{e}_{j} \mid 1 \leq j\right\} \cup\left\{ \pm \mathbf{e}_{j} \pm \mathbf{e}_{i} \mid 0 \leq j<i\right\}, \\
\Delta^{+}=\left\{\mathbf{e}_{j} \mid 1 \leq j\right\} \cup\left\{-\mathbf{e}_{j}+\mathbf{e}_{i}, \mathbf{e}_{j}+\mathbf{e}_{i} \mid 0 \leq j<i\right\},
\end{array}\right. \\
\left\{\begin{array}{l}
\Delta=\left\{ \pm 2 \mathbf{e}_{j} \mid 1 \leq j\right\} \cup\left\{ \pm \mathbf{e}_{j} \pm \mathbf{e}_{i} \mid 0 \leq j<i\right\}, \\
\Delta^{+}=\left\{2 \mathbf{e}_{j} \mid 1 \leq j\right\} \cup\left\{-\mathbf{e}_{j}+\mathbf{e}_{i}, \mathbf{e}_{j}+\mathbf{e}_{i} \mid 0 \leq j<i\right\}
\end{array}\right. \\
\left\{\begin{array}{l}
\Delta=\left\{ \pm \mathbf{e}_{j} \pm \mathbf{e}_{i} \mid 0 \leq j<i\right\}, \\
\Delta^{+}=\left\{-\mathbf{e}_{j}+\mathbf{e}_{i}, \mathbf{e}_{j}+\mathbf{e}_{i} \mid 0 \leq j<i\right\}
\end{array}\right.
\end{gathered}
$$

We set $E:=\bigoplus_{j \in \mathbb{Z}>0} \mathbb{Z} \mathbf{e}_{j} \subset \mathfrak{h}^{*}$ and then $E_{\mathbb{C}}:=\mathbb{C} \otimes_{\mathbb{Z}} E=\bigoplus_{j \in \mathbb{Z}_{>0}} \mathbb{C e}_{j} \subset \mathfrak{h}^{*}$. Note that $\Delta$ is contained in $E$ and that $E$ is stable under the action of the Weyl group $W$. Define a $W$-invariant, symmetric $\mathbb{C}$-bilinear form $(\cdot, \cdot)$ on $E_{\mathbb{C}}$ by $\left(\mathbf{e}_{i}, \mathbf{e}_{j}\right)=\delta_{i j}$ for $i, j \in \mathbb{Z}_{\geq 0}$, and a $\mathbb{C}$-linear isomorphism $\psi: E_{\mathbb{C}}=\bigoplus_{j \in \mathbb{Z}_{\geq 0}} \mathbb{C e}_{j} \rightarrow \mathfrak{h}=\bigoplus_{j \in \mathbb{Z}_{\geq 0}} \mathbb{C e}_{j}^{\prime}$ by $\psi\left(\mathbf{e}_{j}\right)=\mathbf{e}_{j}^{\prime}$ for $j \in \mathbb{Z}_{\geq 0}$. Also, we define the dual root $\beta^{\vee} \in \mathfrak{h}$ of a root $\beta \in \Delta$ by $\beta^{\vee}=2 \psi(\beta) /(\beta, \beta)$. Observe that $\alpha_{i}^{\vee}=h_{i}$ for all $i \in I$.

2.2. Integral weights and their levels. For each $i \in I$, let $\Lambda_{i} \in \mathfrak{h}^{*}$ denote the $i$ th fundamental weight for $\mathfrak{g}$. We should warn the reader that the elements $\Lambda_{i}, i \in I$, are not contained in the subspace $E_{\mathbb{C}}=\bigoplus_{j \in \mathbb{Z}_{\geq 0}} \mathbb{C e}_{j}$ of $\mathfrak{h}^{*}=\prod_{j \in \mathbb{Z} \geq 0} \mathbb{C e}_{j}$, where the vector space $\prod_{j \in \mathbb{Z} \geq 0} \mathbb{C e}_{j}$ is thought of as a certain completion of $\bigoplus_{j \in \mathbb{Z}_{\geq 0}} \mathbb{C e}_{j}$. 
In fact, we have

$$
\begin{aligned}
& \left\{\begin{array}{l}
\Lambda_{0}=\frac{1}{2}\left(\mathbf{e}_{0}+\mathbf{e}_{1}+\mathbf{e}_{2}+\cdots\right), \\
\Lambda_{i}=\mathbf{e}_{i}+\mathbf{e}_{i+1}+\mathbf{e}_{i+2}+\cdots
\end{array} \text { for } i \in \mathbb{Z}_{\geq 1}, \quad \text { if } \mathfrak{g} \text { is of type } B_{\infty},\right. \\
& \Lambda_{i}=\mathbf{e}_{i}+\mathbf{e}_{i+1}+\mathbf{e}_{i+2}+\cdots \quad \text { for } i \in I \quad \text { if } \mathfrak{g} \text { is of type } C_{\infty}, \\
& \left\{\begin{array}{l}
\Lambda_{0}=\frac{1}{2}\left(\mathbf{e}_{0}+\mathbf{e}_{1}+\mathbf{e}_{2}+\cdots\right), \\
\Lambda_{1}=\frac{1}{2}\left(-\mathbf{e}_{0}+\mathbf{e}_{1}+\mathbf{e}_{2}+\cdots\right), \\
\Lambda_{i}=\mathbf{e}_{i}+\mathbf{e}_{i+1}+\mathbf{e}_{i+2}+\cdots
\end{array} \text { for } i \in \mathbb{Z}_{\geq 2},\right.
\end{aligned}
$$

Let $P:=\bigoplus_{i \in I} \mathbb{Z} \Lambda_{i} \subset \mathfrak{h}^{*}$ denote the integral weight lattice for $\mathfrak{g}$, and let $P_{+}:=$ $\sum_{i \in I} \mathbb{Z}_{\geq 0} \Lambda_{i} \subset P$ be the set of dominant integral weights for $\mathfrak{g}$.

For an integral weight $\lambda \in P$, we define $\lambda^{(j)} \in(1 / 2) \mathbb{Z}$ for $j \in \mathbb{Z}_{\geq 0}$ by

$$
\lambda^{(j)}:=\left\langle\lambda, \mathbf{e}_{j}^{\prime}\right\rangle, \quad \text { or equivalently, } \quad \lambda=\lambda^{(0)} \mathbf{e}_{0}+\lambda^{(1)} \mathbf{e}_{1}+\lambda^{(2)} \mathbf{e}_{2}+\cdots .
$$

Note that either $\lambda^{(j)} \in \mathbb{Z}$ for all $j \in \mathbb{Z}_{\geq 0}$ or $\lambda^{(j)} \in 1 / 2+\mathbb{Z}$ for all $j \in \mathbb{Z}_{\geq 0}$. If $\lambda \in P$, then for $n$ sufficiently large, we have $\lambda^{(n)}=\lambda^{(n+1)}=\lambda^{(n+2)}=\cdots$ since $\lambda \in P$ is a finite sum of integer multiples of fundamental weights. In this case, we set $L_{\lambda}:=\lambda^{(n)}$ and call it the level of the integral weight $\lambda$. Note that $E \subset P$ is identical to the set of integral weights of level zero, and that if $\lambda \in P$ is a dominant integral weight not equal to 0 , then the level $L_{\lambda}$ of $\lambda$ is positive.

Remark 2.1 (cf. [Ko2, statements (2)-(4) on p. 82]). Let $\lambda \in P_{+}$. Then, it follows that

$$
0 \leq \underbrace{\left\langle\lambda^{(0)}\right\rangle \leq \lambda^{(1)} \leq \lambda^{(2)} \leq \cdots \leq \lambda^{(n-1)}<}_{\text {If } n=0, \text { then this part is omitted. }} L_{\lambda}=\lambda^{(n)}=\lambda^{(n+1)}=\cdots
$$

for some $n \in \mathbb{Z}_{\geq 0}$, where for $x \in(1 / 2) \mathbb{Z}$, we set

$$
\langle x\rangle:= \begin{cases}x & \text { if } \mathfrak{g} \text { is of type } B_{\infty} \text { or } C_{\infty}, \\ |x| & \text { if } \mathfrak{g} \text { is of type } D_{\infty} .\end{cases}
$$

2.3. Quantized universal enveloping algebras and their finite rank subalgebras. We set $P^{\vee}:=\bigoplus_{i \in I} \mathbb{Z} h_{i} \subset \mathfrak{h}$, and let $U_{q}(\mathfrak{g})=\left\langle x_{i}, y_{i}, q^{h} \mid i \in I, h \in P^{\vee}\right\rangle$ denote the quantized universal enveloping algebra of $\mathfrak{g}$ over $\mathbb{C}(q)$ with integral weight lattice $P$ and Chevalley generators $x_{i}, y_{i}, i \in I$. Also, let $U_{q}^{+}(\mathfrak{g})$ (resp., $\left.U_{q}^{-}(\mathfrak{g})\right)$ denote the positive (resp., negative) part of $U_{q}(\mathfrak{g})$, that is, the $\mathbb{C}(q)$ subalgebra of $U_{q}(\mathfrak{g})$ generated by $x_{i}, i \in I$ (resp., $\left.y_{i}, i \in I\right)$. We have a $\mathbb{C}(q)$-algebra anti-automorphism $*: U_{q}(\mathfrak{g}) \rightarrow U_{q}(\mathfrak{g})$ defined by

$$
\begin{cases}\left(q^{h}\right)^{*}=q^{-h} & \text { for } h \in P^{\vee}, \\ x_{i}^{*}=x_{i}, y_{i}^{*}=y_{i} & \text { for } i \in I .\end{cases}
$$

Note that $U_{q}^{ \pm}(\mathfrak{g})$ is stable under the $\mathbb{C}(q)$-algebra anti-automorphism $*: U_{q}(\mathfrak{g}) \rightarrow$ $U_{q}(\mathfrak{g})$. 
For $m, n \in \mathbb{Z}_{\geq 0}$ with $n \geq m$, we denote the finite interval $\{m, m+1, \ldots, n\}$ in $I=\mathbb{Z}_{\geq 0}$ by $[m, n]$. Also, for $n \in \mathbb{Z}_{\geq 0}$, we simply write $[n]$ for the finite interval $[0, n]=\{0,1, \ldots, n\}$ in $I=\mathbb{Z}_{\geq 0}$. Let $J$ be a finite interval in $I=\mathbb{Z}_{\geq 0}$, which is of the form $[m, n]$ for some $m, n \in \mathbb{Z}_{\geq 0}$ with $n \geq m$. We denote by $\mathfrak{g}_{J}$ the (Lie) subalgebra of $\mathfrak{g}$ generated by $x_{i}, y_{i}, i \in J$, and $\mathfrak{h}$. Then, the set $\Delta_{J}:=$ $\Delta \cap\left(\bigoplus_{i \in J} \mathbb{Z} \alpha_{i}\right)$ and $\Delta_{J}^{+}:=\Delta^{+} \cap\left(\bigoplus_{i \in J} \mathbb{Z} \alpha_{i}\right)$ are the sets of roots and positive roots for $\mathfrak{g}_{J}$, respectively.

Denote by $U_{q}\left(\mathfrak{g}_{J}\right)$ the $\mathbb{C}(q)$-subalgebra of $U_{q}(\mathfrak{g})$ generated by $x_{i}, y_{i}, i \in J$, and $q^{h}$, $h \in P^{\vee}$, which can be thought of as the quantized universal enveloping algebra of $\mathfrak{g}_{J}$ over $\mathbb{C}(q)$. Also, denote by $U_{q}^{+}\left(\mathfrak{g}_{J}\right)$ (resp., $\left.U_{q}^{-}\left(\mathfrak{g}_{J}\right)\right)$ the positive (resp., negative) part of $U_{q}\left(\mathfrak{g}_{J}\right)$, that is, the $\mathbb{C}(q)$-subalgebra of $U_{q}\left(\mathfrak{g}_{J}\right)$ generated by $x_{i}, i \in J$ (resp., $\left.y_{i}, i \in J\right)$. Then it is easily seen that $U_{q}\left(\mathfrak{g}_{J}\right)$ and $U_{q}^{ \pm}\left(\mathfrak{g}_{J}\right)$ are stable under the $\mathbb{C}(q)$-algebra anti-automorphism $*: U_{q}(\mathfrak{g}) \rightarrow U_{q}(\mathfrak{g})$ given by (2.14).

Let $W_{J}$ denote the (finite) subgroup of $W$ generated by the $r_{i}$ for $i \in J$, which is the Weyl group of $\mathfrak{g}_{J}$. An integral weight $\lambda \in P$ is said to be $J$-dominant (resp., $J$-anti-dominant) if $\left\langle\lambda, h_{i}\right\rangle \geq 0$ (resp., $\left\langle\lambda, h_{i}\right\rangle \leq 0$ ) for all $i \in J$. For each integral weight $\lambda \in P$, we denote by $\lambda_{J}$ the unique element of $W_{J} \lambda$ that is $J$-dominant.

Remark 2.2 (cf. [Ko2, statements (2)-(4) on p. 82]). Let $n \in \mathbb{Z}_{\geq 3}$, and let $\lambda \in P$ be an $[n]$-dominant integral weight. Then it follows from (2.4) that $0 \leq\left\langle\lambda^{(0)}\right\rangle \leq$ $\lambda^{(1)} \leq \cdots \leq \lambda^{(n-1)} \leq \lambda^{(n)}$.

Now, for $\lambda \in E=\bigoplus_{j \in \mathbb{Z} \geq 0} \mathbb{Z} \mathbf{e}_{j}$, we set

$$
\operatorname{Supp}(\lambda):=\left\{j \in \mathbb{Z}_{\geq 0} \mid \lambda^{(j)} \neq 0\right\} .
$$

By using (2.5)-(2.7) and Remark 2.2, we can easily show the following lemma.

Lemma 2.3. Let $\lambda \in E$, and set $p:=\# \operatorname{Supp}(\lambda)$. Let $n \in \mathbb{Z}_{\geq 3}$ be such that $\operatorname{Supp}(\lambda) \varsubsetneqq[n]$. Then, the unique $[n]$-dominant element $\lambda_{[n]}$ of $W_{[n]} \lambda$ has the following property:

$$
\operatorname{Supp}\left(\lambda_{[n]}\right)=[n-p+1, n] \quad \text { and } \quad 0<\lambda_{[n]}^{(n-p+1)} \leq \cdots \leq \lambda_{[n]}^{(n-1)} \leq \lambda_{[n]}^{(n)} .
$$

\section{Path model for the CRystal Basis of An extremal Weight module}

3.1. Extremal elements. Let $\mathcal{B}$ be a $U_{q}(\mathfrak{g})$-crystal (resp., $U_{q}\left(\mathfrak{g}_{J}\right)$-crystal for a finite interval $J$ in $I=\mathbb{Z}_{\geq 0}$ ), equipped with the maps $e_{i}, f_{i}: \mathcal{B} \rightarrow \mathcal{B} \cup\{\mathbf{0}\}$ for $i \in I$ (resp., $i \in J$ ), which we call the Kashiwara operators, and the maps wt $: \mathcal{B} \rightarrow P$, $\varepsilon_{i}, \varphi_{i}: \mathcal{B} \rightarrow \mathbb{Z} \cup\{-\infty\}$ for $i \in I$ (resp., $i \in J$ ). Here, $\mathbf{0}$ is a formal element not contained in $\mathcal{B}$. For $\nu \in P$, we denote by $\mathcal{B}_{\nu}$ the subset of $\mathcal{B}$ consisting of all elements of weight $\nu$.

Definition 3.1 (cf. [Kas3, p. 389]). (1) A $U_{q}(\mathfrak{g})$-crystal $\mathcal{B}$ is said to be normal if $\mathcal{B}$, regarded as a $U_{q}\left(\mathfrak{g}_{K}\right)$-crystal by restriction, is isomorphic to a direct sum of the crystal bases of finite-dimensional irreducible $U_{q}\left(\mathfrak{g}_{K}\right)$-modules for every finite interval $K$ in $I=\mathbb{Z}_{\geq 0}$.

(2) Let $J$ be a finite interval in $I=\mathbb{Z}_{\geq 0}$. A $U_{q}\left(\mathfrak{g}_{J}\right)$-crystal $\mathcal{B}$ is said to be normal if $\mathcal{B}$, regarded as a $U_{q}\left(\mathfrak{g}_{K}\right)$-crystal by restriction, is isomorphic to a direct sum of the crystal bases of finite-dimensional irreducible $U_{q}\left(\mathfrak{g}_{K}\right)$-modules for every finite interval $K$ in $I=\mathbb{Z}_{\geq 0}$ contained in $J$. 
If $\mathcal{B}$ is a normal $U_{q}(\mathfrak{g})$-crystal, then $\mathcal{B}$, regarded as a $U_{q}\left(\mathfrak{g}_{J}\right)$-crystal by restriction, is a normal $U_{q}\left(\mathfrak{g}_{J}\right)$-crystal for every finite interval $J$ in $I=\mathbb{Z}_{\geq 0}$. Also, if $\mathcal{B}$ is a normal $U_{q}(\mathfrak{g})$-crystal (resp., a normal $U_{q}\left(\mathfrak{g}_{J}\right)$-crystal for a finite interval $J$ in $\left.I=\mathbb{Z}_{\geq 0}\right)$, then we have

$$
\varepsilon_{i}(b)=\max \left\{k \in \mathbb{Z}_{\geq 0} \mid e_{i}^{k} b \neq \mathbf{0}\right\} \quad \text { and } \quad \varphi_{i}(b)=\max \left\{k \in \mathbb{Z}_{\geq 0} \mid f_{i}^{k} b \neq \mathbf{0}\right\}
$$

for $b \in \mathcal{B}$ and $i \in I$ (resp., $i \in J$ ). In this case, we set

$$
e_{i}^{\max } b:=e_{i}^{\varepsilon_{i}(b)} b \quad \text { and } \quad f_{i}^{\max } b:=f_{i}^{\varphi_{i}(b)} b
$$

for $b \in \mathcal{B}$ and $i \in I$ (resp., $i \in J$ ). Also, we can define an action of the Weyl group $W$ (resp., $W_{J}$ ) on $\mathcal{B}$ as follows. For each $i \in I$ (resp., $i \in J$ ), define $S_{i}: \mathcal{B} \rightarrow \mathcal{B}$ by

$$
S_{i} b=\left\{\begin{array}{ll}
f_{i}^{k} b & \text { if } k:=\left\langle\mathrm{wt} b, h_{i}\right\rangle \geq 0, \\
e_{i}^{-k} b & \text { if } k:=\left\langle\mathrm{wt} b, h_{i}\right\rangle<0,
\end{array} \quad \text { for } b \in \mathcal{B} .\right.
$$

Then, these operators $S_{i}, i \in I$, give rise to a unique (well-defined) action $S: W \rightarrow$ $\operatorname{Bij}(\mathcal{B})\left(\operatorname{resp} ., S: W_{J} \rightarrow \operatorname{Bij}(\mathcal{B})\right), w \mapsto S_{w}$, of the Weyl group $W$ (resp., $W_{J}$ ) on the set $\mathcal{B}$ such that $S_{r_{i}}=S_{i}$ for all $i \in I$ (resp., $i \in J$ ). Here, for a set $X, \operatorname{Bij}(X)$ denotes the group of all bijections from the set $X$ to itself.

Definition 3.2. Suppose that $\mathcal{B}$ is a normal $U_{q}(\mathfrak{g})$-crystal (resp., a $U_{q}\left(\mathfrak{g}_{J}\right)$-crystal for a finite interval $J$ in $I=\mathbb{Z}_{\geq 0}$ ).

(1) An element $b \in \mathcal{B}$ is said to be extremal (resp., $J$-extremal) if for every $w \in W$ and $i \in I$ (resp., $w \in W_{J}$ and $i \in J$ ),

$$
\begin{cases}e_{i} S_{w} b=\mathbf{0} & \text { if }\left\langle w(\mathrm{wt} b), h_{i}\right\rangle \geq 0 \\ f_{i} S_{w} b=\mathbf{0} & \text { if }\left\langle w(\mathrm{wt} b), h_{i}\right\rangle \leq 0 .\end{cases}
$$

(2) An element $b \in \mathcal{B}$ is said to be maximal (resp., $J$-maximal) if $e_{i} b=\mathbf{0}$ for all $i \in I$ (resp., $i \in J$ ). Also, an element $b \in \mathcal{B}$ is said to be minimal (resp., $J$-minimal) if $f_{i} b=\mathbf{0}$ for all $i \in I$ (resp., $i \in J$ ).

3.2. Crystal bases of extremal weight modules. In this subsection, we study some basic properties of crystal bases of extremal weight modules. Note that all results in [Kas1] Kas4] about extremal weight modules and their crystal bases that we use in this paper remain valid in the case of infinite rank affine Lie algebras.

Definition 3.3 (see Kas3, Definition 8.1]). Let $\lambda \in P$ be an integral weight.

(1) Let $M$ be an integrable $U_{q}(\mathfrak{g})$-module. A weight vector $v \in M$ of weight $\lambda$ is said to be extremal if there exists a family $\left\{v_{w}\right\}_{w \in W}$ of weight vectors in $M$ satisfying the following conditions:

(i) If $w$ is the identity element $e$ of $W$, then $v_{w}=v_{e}=v$;

(ii) for $w \in W$ and $i \in I$ such that $k:=\left\langle w \lambda, h_{i}\right\rangle \geq 0$, we have $x_{i} v_{w}=0$ and $y_{i}^{(k)} v_{w}=v_{r_{i} w}$;

(iii) for $w \in W$ and $i \in I$ such that $k:=\left\langle w \lambda, h_{i}\right\rangle \leq 0$, we have $y_{i} v_{w}=0$ and $x_{i}^{(-k)} v_{w}=v_{r_{i} w}$.

Here, for $i \in I$ and $k \in \mathbb{Z}_{\geq 0}, x_{i}^{(k)}$ and $y_{i}^{(k)}$ denote the $k$-th $q$-divided powers of $x_{i}$ and $y_{i}$, respectively.

(2) Let $J$ be a finite interval in $I=\mathbb{Z}_{\geq 0}$, and let $M$ be an integrable $U_{q}\left(\mathfrak{g}_{J}\right)$ module. A weight vector $v \in M$ of weight $\bar{\lambda}$ is said to be $J$-extremal if there exists 
a family $\left\{v_{w}\right\}_{w \in W_{J}}$ of weight vectors in $M$ satisfying the same conditions as (i), (ii), (iii) above, with $W$ replaced by $W_{J}$ and $I$ by $J$.

The extremal weight $U_{q}(\mathfrak{g})$-module $V(\lambda)$ of extremal weight $\lambda$ is, by definition, the integrable $U_{q}(\mathfrak{g})$-module generated by a single element $v_{\lambda}$ subject to the defining relation that the $v_{\lambda}$ is an extremal vector of weight $\lambda$ (see [Kas3, Proposition 8.2.2] and also [Kas5, §3.1]). We know from [Kas3, Proposition 8.2.2] that $V(\lambda)$ admits the crystal basis $(\mathcal{L}(\lambda), \mathcal{B}(\lambda))$ and that $\mathcal{B}(\lambda)$ is a normal $U_{q}(\mathfrak{g})$-crystal. If we denote by $u_{\lambda} \in \mathcal{B}(\lambda)$ the element corresponding to the extremal vector $v_{\lambda} \in V(\lambda)$ of weight $\lambda$, then the element $u_{\lambda} \in \mathcal{B}(\lambda)$ is extremal.

Remark 3.4 (see [Kas3, $\S \S 8.2$ and 8.3]). (1) If $\lambda \in P_{+}$, then the extremal weight module $V(\lambda)$ is isomorphic to the irreducible highest weight $U_{q}(\mathfrak{g})$-module of highest weight $\lambda$. Therefore, the crystal basis $\mathcal{B}(\lambda)$ is isomorphic, as a crystal, to the crystal basis of the irreducible highest weight $U_{q}(\mathfrak{g})$-module of highest weight $\lambda$.

(2) For each $w \in W$, there exists an isomorphism $V(\lambda) \cong V(w \lambda)$ of $U_{q}(\mathfrak{g})$ modules between $V(\lambda)$ and $V(w \lambda)$. Also, for each $w \in W$, there exists an isomorphism $\mathcal{B}(\lambda) \cong \mathcal{B}(w \lambda)$ of $U_{q}(\mathfrak{g})$-crystals between $\mathcal{B}(\lambda)$ and $\mathcal{B}(w \lambda)$.

Let $J$ be a finite interval in $I=\mathbb{Z}_{\geq 0}$. As in the case of $V(\lambda)$, the extremal weight $U_{q}\left(\mathfrak{g}_{J}\right)$-module $V_{J}(\lambda)$ of extremal weight $\lambda$ is, by definition, the integrable $U_{q}\left(\mathfrak{g}_{J}\right)$-module generated by a single element $v_{\lambda}$ subject to the defining relation that the $v_{\lambda}$ is a $J$-extremal vector of weight $\lambda$. We denote by $\left(\mathcal{L}_{J}(\lambda), \mathcal{B}_{J}(\lambda)\right)$ the crystal basis of $V_{J}(\lambda)$.

Remark 3.5. Let $J$ be a finite interval in $I=\mathbb{Z}_{>0}$. Then, results entirely similar to those in Remark 3.4 hold for extremal weight $U_{q}\left(\mathfrak{g}_{J}\right)$-modules and their crystal bases. If $\lambda$ is $J$-dominant (resp., $J$-anti-dominant), then $V_{J}(\lambda)$ is the finitedimensional irreducible $U_{q}\left(\mathfrak{g}_{J}\right)$-module of highest weight $\lambda$ (resp., lowest weight $\lambda$ ). Also, for $\lambda \in P$, we have $\mathcal{B}_{J}(\lambda) \cong \mathcal{B}_{J}\left(\lambda_{J}\right)$ as $U_{q}\left(\mathfrak{g}_{J}\right)$-crystals since $\lambda_{J} \in W_{J} \lambda$. Because $\mathcal{B}_{J}\left(\lambda_{J}\right)$ is isomorphic, as a $U_{q}\left(\mathfrak{g}_{J}\right)$-crystal, to the crystal basis of the finitedimensional irreducible $U_{q}\left(\mathfrak{g}_{J}\right)$-module of highest weight $\lambda_{J}$, it follows that the crystal graph of $\mathcal{B}_{J}(\lambda)$ is connected and that $\#\left(\mathcal{B}_{J}(\lambda)\right)_{\nu}=1$ for all $\nu \in W_{J} \lambda$.

To prove Proposition 3.6 below, we need to recall the description of the crystal basis $\mathcal{B}(\lambda)$ for $\lambda \in P$ from Kas3. Let $(\mathcal{L}( \pm \infty), \mathcal{B}( \pm \infty))$ denote the crystal basis of $U_{q}^{\mp}(\mathfrak{g})$. Recall from [Kas1, Proposition 5.2.4] that the crystal lattice $\mathcal{L}( \pm \infty)$ of $U_{q}^{\mp}(\mathfrak{g})$ is stable under the $\mathbb{C}(q)$-algebra anti-automorphism $*: U_{q}(\mathfrak{g}) \rightarrow$ $U_{q}(\mathfrak{g})$ given by (2.14). Furthermore, we know from [Kas2, Theorem 2.1.1] that the $\mathbb{C}$-linear automorphism (also denoted by $*$ ) on $\mathcal{L}( \pm \infty) / q \mathcal{L}( \pm \infty)$ induced by * : $\mathcal{L}( \pm \infty) \rightarrow \mathcal{L}( \pm \infty)$ stabilizes the crystal basis $\mathcal{B}( \pm \infty)$ and gives rise to a map $*: \mathcal{B}( \pm \infty) \rightarrow \mathcal{B}( \pm \infty)$. Now, we set $\mathcal{B}^{\lambda}:=\mathcal{B}(\infty) \otimes \mathcal{T}_{\lambda} \otimes \mathcal{B}(-\infty)$ for $\lambda \in P$, where $\mathcal{T}_{\lambda}:=\left\{t_{\lambda}\right\}$ is a $U_{q}(\mathfrak{g})$-crystal consisting of a single element $t_{\lambda}$ of weight $\lambda \in P$ (see [Kas3, Example 1.5.3, part 2]), and then set $\widetilde{\mathcal{B}}:=\bigoplus_{\lambda \in P} \mathcal{B}^{\lambda}$. Also, we define a map $*: \widetilde{\mathcal{B}} \rightarrow \widetilde{\mathcal{B}}$ by $\left(b_{1} \otimes t_{\lambda} \otimes b_{2}\right)^{*}=b_{1}^{*} \otimes t_{-\left(\mathrm{wt} b_{1}+\lambda+\mathrm{wt} b_{2}\right)} \otimes b_{2}^{*}$ for $b_{1} \in \mathcal{B}(\infty)$, $\lambda \in P$, and $b_{2} \in \mathcal{B}(-\infty)$ (cf. Kas3, Corollary 4.3.3]). It follows from Kas3, Theorem 3.1.1 and Theorem 2.1.2(v)] that $\mathcal{B}^{\lambda}$ is normal for all $\lambda \in P$, and hence that so is $\widetilde{\mathcal{B}}=\bigoplus_{\lambda \in P} \mathcal{B}^{\lambda}$. Moreover, by [Kas3, Proposition 8.2.2, Theorem 3.1.1, and Corollary 4.3.3], the subset

$$
\left\{b \in \mathcal{B}^{\lambda} \mid b^{*} \text { is extremal }\right\} \subset \mathcal{B}^{\lambda}
$$


is a $U_{q}(\mathfrak{g})$-subcrystal of $\mathcal{B}^{\lambda}$, and it is isomorphic, as a $U_{q}(\mathfrak{g})$-crystal, to the crystal basis $\mathcal{B}(\lambda)$. Under this isomorphism, the extremal element $u_{\lambda} \in \mathcal{B}(\lambda)$ corresponds to the element $u_{\infty} \otimes t_{\lambda} \otimes u_{-\infty} \in \mathcal{B}^{\lambda}$, where $u_{ \pm \infty}$ is the element of $\mathcal{B}( \pm \infty)$ corresponding to the identity element $1 \in U_{q}^{\mp}(\mathfrak{g})$. Thus, we can identify $\mathcal{B}(\lambda)$ with the $U_{q}(\mathfrak{g})$ subcrystal given by (3.3) and $u_{\lambda} \in \mathcal{B}(\lambda)$ with $u_{\infty} \otimes t_{\lambda} \otimes u_{-\infty} \in \mathcal{B}^{\lambda}$.

We have the following proposition (see also [Kw2, Proposition 3.1] in type $A_{+\infty}$ and [Kw3, Proposition 4.1] in type $\left.A_{\infty}\right)$.

Proposition 3.6. Let $\lambda \in P$ be an integral weight. Then, the crystal graph of the crystal basis $\mathcal{B}(\lambda)$ is connected.

Proof. While the argument in the proof of [Kw2, Proposition 3.1] in type $A_{+\infty}$ (or, the one of [Kw3, Proposition 4.1] in type $A_{\infty}$ ) still works in the case of type $B_{\infty}$, $C_{\infty}$, or $D_{\infty}$, we prefer to give a different proof.

For each $n \in \mathbb{Z}_{\geq 0}$, let $\left(\mathcal{L}_{[n]}( \pm \infty), \mathcal{B}_{[n]}( \pm \infty)\right)$ denote the crystal basis of $U_{q}^{\mp}\left(\mathfrak{g}_{[n]}\right)$ $\subset U_{q}^{\mp}(\mathfrak{g})$. We deduce from the definitions that $U_{q}^{\mp}\left(\mathfrak{g}_{[n]}\right)$ is stable under the Kashiwara operators $e_{i}$ and $f_{i}$ for $i \in[n]$ on $U_{q}^{\mp}(\mathfrak{g})$ and that their restrictions to $U_{q}^{\mp}\left(\mathfrak{g}_{[n]}\right)$ are exactly the Kashiwara operators $e_{i}$ and $f_{i}$ for $i \in[n]$ on $U_{q}^{\mp}\left(\mathfrak{g}_{[n]}\right)$, respectively. Therefore, the crystal lattice $\mathcal{L}_{[n]}( \pm \infty)$ of $U_{q}^{\mp}\left(\mathfrak{g}_{[n]}\right)$ is identical to the $A$-submodule of the crystal lattice $\mathcal{L}( \pm \infty)$ of $U_{q}^{\mp}(\mathfrak{g})$ generated by those elements of the form $X \cdot 1 \in U_{q}^{\mp}\left(\mathfrak{g}_{[n]}\right) \subset U_{q}\left(\mathfrak{g}_{[n]}\right)$ for some monomial $X$ in the Kashiwara operators $e_{i}$ and $f_{i}$ for $i \in[n]$, where $A:=\{f(q) \in \mathbb{C}(q) \mid f(q)$ is regular at $q=0\}$. Consequently, the crystal basis $\mathcal{B}_{[n]}( \pm \infty)$ of $U_{q}^{\mp}\left(\mathfrak{g}_{[n]}\right)$ is identical to the subset of $\mathcal{B}( \pm \infty)$ consisting of all elements $b$ of the form $b=X u_{ \pm \infty}$ for some monomial $X$ in the Kashiwara operators $e_{i}$ and $f_{i}$ for $i \in[n]$.

Now, we define a subset $\mathcal{B}_{[n]}^{\lambda}$ of $\mathcal{B}^{\lambda}$ by

$$
\mathcal{B}_{[n]}^{\lambda}=\left\{b_{1} \otimes t_{\lambda} \otimes b_{2} \in \mathcal{B}^{\lambda} \mid b_{1} \in \mathcal{B}_{[n]}(\infty), b_{2} \in \mathcal{B}_{[n]}(-\infty)\right\} .
$$

Then, it is obvious from the tensor product rule for crystals that $\mathcal{B}_{[n]}^{\lambda}$ is a $U_{q}\left(\mathfrak{g}_{[n]}\right)$ crystal isomorphic to the tensor product $\mathcal{B}_{[n]}(\infty) \otimes \mathcal{T}_{\lambda} \otimes \mathcal{B}_{[n]}(-\infty)$ of $U_{q}\left(\mathfrak{g}_{[n]}\right)$ crystals. Namely, as $U_{q}\left(\mathfrak{g}_{[n]}\right)$-crystals,

$$
\mathcal{B}_{[n]}^{\lambda} \stackrel{\sim}{\rightarrow} \mathcal{B}_{[n]}(\infty) \otimes \mathcal{T}_{\lambda} \otimes \mathcal{B}_{[n]}(-\infty), \quad b_{1} \otimes t_{\lambda} \otimes b_{2} \mapsto b_{1} \otimes t_{\lambda} \otimes b_{2}
$$

Hence, by [Kas3, Theorem 3.1.1 and Theorem 2.1.2(v)], $\mathcal{B}_{[n]}^{\lambda}$ is a normal $U_{q}\left(\mathfrak{g}_{[n]}\right)$ crystal. Also, note that

$$
\mathcal{B}(\lambda) \cap \mathcal{B}_{[n]}^{\lambda} \subset \mathcal{B}(\lambda) \cap \mathcal{B}_{[n+1]}^{\lambda} \quad \text { for all } n \in \mathbb{Z}_{\geq 0} \text {, and } \mathcal{B}(\lambda)=\bigcup_{n \geq 0}\left(\mathcal{B}(\lambda) \cap \mathcal{B}_{[n]}^{\lambda}\right) .
$$

We claim that the crystal basis $\mathcal{B}_{[n]}(\lambda)$ of the extremal weight $U_{q}\left(\mathfrak{g}_{[n]}\right)$-module of extremal weight $\lambda$ is isomorphic, as a $U_{q}\left(\mathfrak{g}_{[n]}\right)$-crystal, to $\mathcal{B}(\lambda) \cap \mathcal{B}_{[n]}^{\lambda}$. Indeed, it follows from [Kas3, Proposition 8.2.2, Theorem 3.1.1, and Corollary 4.3.3], together with (3.4), that the subset

$$
\left\{b \in \mathcal{B}_{[n]}^{\lambda} \mid b^{*} \text { is }[n] \text {-extremal }\right\} \quad\left(\subset \mathcal{B}_{[n]}^{\lambda} \cong \mathcal{B}_{[n]}(\infty) \otimes \mathcal{T}_{\lambda} \otimes \mathcal{B}_{[n]}(-\infty)\right)
$$

is a $U_{q}\left(\mathfrak{g}_{[n]}\right)$-subcrystal of $\mathcal{B}_{[n]}^{\lambda}$ and that it is isomorphic, as a $U_{q}\left(\mathfrak{g}_{[n]}\right)$-crystal, to the crystal basis $\mathcal{B}_{[n]}(\lambda)$. Therefore, we obtain an embedding of $U_{q}\left(\mathfrak{g}_{[n]}\right)$-crystals from $\mathcal{B}(\lambda) \cap \mathcal{B}_{[n]}^{\lambda}$ into $\mathcal{B}_{[n]}(\lambda)$. Because we know from Remark 3.5 with $J=[n]$ that 
the crystal graph of the $U_{q}\left(\mathfrak{g}_{[n]}\right)$-crystal $\mathcal{B}_{[n]}(\lambda)$ is connected, we conclude that the embedding $\mathcal{B}(\lambda) \cap \mathcal{B}_{[n]}^{\lambda} \hookrightarrow \mathcal{B}_{[n]}(\lambda)$ above is an isomorphism of $U_{q}\left(\mathfrak{g}_{[n]}\right)$-crystals.

Let us take $b_{1}, b_{2} \in \mathcal{B}(\lambda)$ arbitrarily. We show that there exists a monomial $X$ in the Kashiwara operators $e_{i}$ and $f_{i}$ for $i \in I$ such that $X b_{1}=b_{2}$. It follows from (3.5) that there exists $n \in \mathbb{Z}_{\geq 0}$ such that $b_{1}$ and $b_{2}$ are both contained in $\mathcal{B}(\lambda) \cap \mathcal{B}_{[n]}^{\lambda}$. Since $\mathcal{B}(\lambda) \cap \mathcal{B}_{[n]}^{\lambda} \cong \mathcal{B}_{[n]}(\lambda)$ as $U_{q}\left(\mathfrak{g}_{[n]}\right)$-crystals by the "claim" above, we deduce from Remark 3.5 with $J=[n]$ that there exists a monomial $X$ in the Kashiwara operators $e_{i}$ and $f_{i}$ for $i \in[n]$ such that $X b_{1}=b_{2}$. This finishes the proof of Proposition 3.6 .

Proposition 3.7. Let $\lambda \in P$ be an integral weight. For each $w \in W$, we have $\mathcal{B}(\lambda)_{w \lambda}=\left\{S_{w} u_{\lambda}\right\}$.

Proof. By using the action of the Weyl group $W$ on $\mathcal{B}(\lambda)$, we are reduced to the case $w=e$. Namely, it suffices to show that $\mathcal{B}(\lambda)_{\lambda}=\left\{u_{\lambda}\right\}$. Let $b \in \mathcal{B}(\lambda)_{\lambda}$. Then, there exists $n \in \mathbb{Z}_{\geq 0}$ such that $b \in \mathcal{B}(\lambda) \cap \mathcal{B}_{[n]}^{\lambda}$ by (3.5). Note that $u_{\lambda} \in \mathcal{B}(\lambda) \cap \mathcal{B}_{[n]}^{\lambda}$. Since $\mathcal{B}(\lambda) \cap \mathcal{B}_{[n]}^{\lambda} \cong \mathcal{B}_{[n]}(\lambda)$ as $U_{q}\left(\mathfrak{g}_{[n]}\right)$-crystals by the "claim" in the proof of Proposition 3.6] it follows from Remark 3.5 with $J=[n]$ that $\#\left(\mathcal{B}(\lambda) \cap \mathcal{B}_{[n]}^{\lambda}\right)_{\nu}=1$ for every $\nu \in W_{[n]} \lambda$. In particular, we have $\#\left(\mathcal{B}(\lambda) \cap \mathcal{B}_{[n]}^{\lambda}\right)_{\lambda}=1$, which implies that $b=u_{\lambda}$, as desired.

Remark 3.8. For a general $\xi \in P$, the set $\mathcal{B}(\lambda)_{\xi}$ is not necessarily of finite cardinality.

Proposition 3.9. Let $\lambda, \mu \in P$ be integral weights (not necessarily dominant). Then, $\mathcal{B}(\lambda) \cong \mathcal{B}(\mu)$ as $U_{q}(\mathfrak{g})$-crystals if and only if $\lambda \in W \mu$.

Proof. We know from Remark 3.4 (2) that if $\lambda \in W \mu$, then $\mathcal{B}(\lambda) \cong \mathcal{B}(\mu)$ as $U_{q}(\mathfrak{g})$ crystals. Conversely, suppose that $\mathcal{B}(\lambda) \cong \mathcal{B}(\mu)$ as $U_{q}(\mathfrak{g})$-crystals. Let $b \in \mathcal{B}(\lambda)$ be the element corresponding to $u_{\mu} \in \mathcal{B}(\mu)$ under the isomorphism $\mathcal{B}(\lambda) \cong \mathcal{B}(\mu)$ of $U_{q}(\mathfrak{g})$-crystals. Note that $b \in \mathcal{B}(\lambda)$ is an extremal element of weight $\mu$ since $u_{\mu}$ is as well. Take $n \in \mathbb{Z}_{\geq 0}$ such that $b \in \mathcal{B}(\lambda) \cap \mathcal{B}_{[n]}^{\lambda}$, and let $w \in W_{[n]}$ be such that $w \mu=\mu_{[n]}$ (recall that $\mu_{[n]}$ is the unique element of $W_{[n]} \mu$ that is [n]-dominant). Then, since $S_{w}$ is defined to be a certain composite of the Kashiwara operators $e_{i}$ and $f_{i}$ for $i \in[n]$, we see that $S_{w} b$ is contained in $\mathcal{B}(\lambda) \cap \mathcal{B}_{[n]}^{\lambda}$. Furthermore, since $b \in \mathcal{B}(\lambda)$ is an extremal element of weight $\mu$ and since $\operatorname{wt}\left(S_{w} b\right)=w \mu=\mu_{[n]}$ is $[n]$-dominant, we deduce from Definition $3.2(1)$ that $S_{w} b \in \mathcal{B}(\lambda) \cap \mathcal{B}_{[n]}^{\lambda}$ is an $[n]$ maximal element. Now we recall that, by the "claim" in the proof of Proposition 3.6 and by Remark 3.5 with $J=[n]$, the $U_{q}\left(\mathfrak{g}_{[n]}\right)$-crystal $\mathcal{B}(\lambda) \cap \mathcal{B}_{[n]}^{\lambda}$ is isomorphic to the crystal basis of the finite-dimensional irreducible $U_{q}\left(\mathfrak{g}_{[n]}\right)$-module of highest weight $\lambda_{[n]}$. Therefore, we deduce that the element $S_{w} b$ is the (unique) $[n]$-maximal element of $\mathcal{B}(\lambda) \cap \mathcal{B}_{[n]}^{\lambda}$ of weight $\lambda_{[n]}$ and hence that $\mu_{[n]}=w \mu=\operatorname{wt}\left(S_{w} b\right)=\lambda_{[n]} \in W_{[n]} \lambda$, which implies that $\lambda \in W \mu$. This proves the proposition.

3.3. Lakshmibai-Seshadri paths and the crystal structure on them. In this subsection, following [Li1] and [Li2], we review basic facts about LakshmibaiSeshadri paths and the crystal structure on them. It is easily checked that all results in Li1 and Li2 that we use in this paper remain valid in the case of infinite rank affine Lie algebras. We take and fix an arbitrary (not necessarily dominant) integral weight $\lambda \in P$. 
Definition 3.10. Let $\mu, \nu$ be elements of $W \lambda$. We write $\mu>\nu$ if there exist a sequence $\mu=\xi_{0}, \xi_{1}, \ldots, \xi_{l}=\nu$ of elements of $W \lambda$ and a sequence $\beta_{1}, \ldots, \beta_{l} \in \Delta^{+}$ of positive roots for $\mathfrak{g}$, with $l \geq 1$, such that $\xi_{m}=r_{\beta_{m}}\left(\xi_{m-1}\right)$ and $\left\langle\xi_{m-1}, \beta_{m}^{\vee}\right\rangle \in \mathbb{Z}_{<0}$ for all $1 \leq m \leq l$, where $r_{\beta} \in W$ denotes the reflection with respect to a $\operatorname{root} \beta \in \Delta$. In this case, the sequence $\xi_{0}, \xi_{1}, \ldots, \xi_{l}$ above is called a chain for $(\mu, \nu)$ in $W \lambda$. If $\mu>\nu$, then we define $\operatorname{dist}(\mu, \nu)$ to be the maximum length $l$ of all possible chains for $(\mu, \nu)$ in $W \lambda$.

Remark 3.11. Let $\mu, \nu$ be elements of $W \lambda$ such that $\mu>\nu$. If $\mu=\xi_{0}, \xi_{1}, \ldots, \xi_{l}=\nu$ is a chain for $(\mu, \nu)$ in $W \lambda$, with corresponding positive roots $\beta_{1}, \ldots, \beta_{l} \in \Delta^{+}$, then we have

$$
\nu-\mu \in \sum_{m=1}^{l} \mathbb{Z}_{>0} \beta_{m} \subset \sum_{i \in I} \mathbb{Z}_{\geq 0} \alpha_{i} \backslash\{0\} .
$$

Definition 3.12. Let $\mu, \nu$ be elements of $W \lambda$ such that $\mu>\nu$, and let $0<a<1$ be a rational number. An $a$-chain for $(\mu, \nu)$ in $W \lambda$ is, by definition, a sequence $\mu=$ $\zeta_{0}>\zeta_{1}>\cdots>\zeta_{l}=\nu$ of elements of $W \lambda$, with $l \geq 1$, such that $\operatorname{dist}\left(\zeta_{m-1}, \zeta_{m}\right)=1$ and $\left\langle\zeta_{m-1}, \beta_{m}^{\vee}\right\rangle \in a^{-1} \mathbb{Z}_{<0}$ for all $1 \leq m \leq l$, where $\beta_{m} \in \Delta^{+}$is the positive root for $\mathfrak{g}$ corresponding to a chain for $\left(\zeta_{m-1}, \zeta_{m}\right)$ in $W \lambda$.

Definition $3.13([\mathrm{Li} 2, \S 4])$. Let $\lambda \in P$, and let $(\underline{\nu} ; \underline{a})$ be a pair of a sequence $\underline{\nu}$ : $\nu_{1}>\nu_{2}>\cdots>\nu_{s}$ of elements of $W \lambda$ and a sequence $\underline{a}: 0=a_{0}<a_{1}<\cdots<a_{s}=1$ of rational numbers, with $s \geq 1$. The pair $(\underline{\nu} ; \underline{a})$ is called a Lakshmibai-Seshadri path (LS path for short) of shape $\lambda$ (for $\mathfrak{g}$ ) if for every $u=1,2, \ldots, s-1$, there exists an $a_{u}$-chain for $\left(\nu_{u}, \nu_{u+1}\right)$ in $W \lambda$. We denote by $\mathbb{B}(\lambda)$ the set of all LS paths of shape $\lambda$.

Remark 3.14. (1) It is easily seen from the definition that $\pi_{\nu}:=(\nu ; 0,1) \in \mathbb{B}(\lambda)$ for all $\nu \in W \lambda$.

(2) It is obvious from the definitions that $\mathbb{B}(w \lambda)=\mathbb{B}(\lambda)$ for all $w \in W$.

Let $J$ be a finite interval in $I=\mathbb{Z}_{\geq 0}$. We define an LS path of shape $\lambda$ for $\mathfrak{g}_{J}$ as follows. First we introduce a partial order $>_{J}$ on $W_{J} \lambda$, which is defined as in Definition 3.10, with $W$ replaced by $W_{J}$ and $\Delta^{+}$by $\Delta_{J}^{+}=\Delta^{+} \cap \bigoplus_{i \in J} \mathbb{Z} \alpha_{i}$. If $\mu, \nu \in W_{J} \lambda$ are such that $\mu>_{J} \nu$, then we denote by $\operatorname{dist}_{J}(\cdot, \cdot)$ the maximal length of all possible chains for $(\mu, \nu)$ in $W_{J} \lambda$. Next, for a rational number $0<a<1$, we define $a$-chains in $W_{J} \lambda$ as in Definition 3.12, with $W$ replaced by $W_{J}, \operatorname{dist}(\cdot, \cdot)$ by $\operatorname{dist}_{J}(\cdot, \cdot)$, and $\Delta^{+}$by $\Delta_{J}^{+}$. Finally, we define an LS path of shape $\lambda$ for $\mathfrak{g}_{J}$ in the same way as in Definition 3.13 , with $W$ replaced by $W_{J}$. We denote by $\mathbb{B}_{J}(\lambda)$ the set of all LS paths of shape $\lambda$ for $\mathfrak{g}_{J}$.

The proof of the following lemma is easy. We need only observe that the partial order $>_{J}$ on $W_{J} \lambda$ coincides with the restriction of the partial order $>$ on $W \lambda$ to $W_{J} \lambda$ and that if $\mu, \nu \in W_{J} \lambda$ are such that $\mu>\nu$ (or equivalently, $\mu>_{J} \nu$ ), then $\operatorname{dist}(\mu, \nu)=\operatorname{dist}_{J}(\mu, \nu)$.

Lemma 3.15. Let $J$ be a finite interval in $I=\mathbb{Z}_{\geq 0}$. Then, the set $\mathbb{B}_{J}(\lambda)$ is identical to the subset of $\mathbb{B}(\lambda)$ consisting of all elements $\pi=\left(\nu_{1}, \nu_{2}, \ldots, \nu_{s} ; a_{0}, a_{1}, \ldots, a_{s}\right)$, with $s \geq 1$, satisfying the condition that $\nu_{u} \in W_{J} \lambda$ for all $1 \leq u \leq s$.

It follows from Lemma 3.15 that

$$
\mathbb{B}_{[n]}(\lambda) \subset \mathbb{B}_{[n+1]}(\lambda) \quad \text { for all } n \in \mathbb{Z}_{\geq 0} \text {, and } \quad \mathbb{B}(\lambda)=\bigcup_{n \geq 0} \mathbb{B}_{[n]}(\lambda) .
$$


Now, for real numbers $a, b \in \mathbb{R}$ with $a \leq b$, we set $[a, b]_{\mathbb{R}}:=\{t \in \mathbb{R} \mid a \leq t \leq b\}$. A path is, by definition, a piecewise linear, continuous map $\pi:[0,1]_{\mathbb{R}} \rightarrow \mathbb{R} \otimes_{\mathbb{Z}} P$ from $[0,1]_{\mathbb{R}}$ to $\mathbb{R} \otimes_{\mathbb{Z}} P$ such that $\pi(0)=0$. Let $\pi=(\underline{\nu} ; \underline{a})$ be a pair of a sequence $\underline{\nu}: \nu_{1}, \nu_{2}, \ldots, \nu_{s}$ of integral weights in $P$ and a sequence $\underline{a}: 0=a_{0}<a_{1}<\cdots<$ $a_{s}=1$ of rational numbers. We associate to the pair $\pi=(\underline{\nu} ; \underline{a})$ the following path $\pi:[0,1]_{\mathbb{R}} \rightarrow \mathbb{R} \otimes_{\mathbb{Z}} P:$

$$
\pi(t)=\sum_{v=1}^{u-1}\left(a_{v}-a_{v-1}\right) \nu_{v}+\left(t-a_{u-1}\right) \nu_{u} \quad \text { for } \quad a_{u-1} \leq t \leq a_{u}, 1 \leq u \leq s .
$$

It is easily seen that for pairs $\pi=(\underline{\nu} ; \underline{a}) \in \mathbb{B}(\lambda)$ and $\pi^{\prime}=\left(\underline{\nu}^{\prime} ; \underline{a^{\prime}}\right) \in \mathbb{B}(\lambda), \pi$ is identical to $\pi^{\prime}$ (i.e., $\underline{\nu}=\underline{\nu}^{\prime}$ and $\underline{a}=\underline{a}^{\prime}$ ) if and only if $\pi(t)=\pi^{\prime}(t)$ for all $t \in[0,1]_{\mathbb{R}}$. Hence we can identify the set $\mathbb{B}(\lambda)$ of all LS paths of shape $\lambda$ with the set of corresponding paths via (3.8). Note that the element $\pi_{\nu}=(\nu ; 0,1) \in \mathbb{B}(\lambda)$ for $\nu \in W \lambda$ corresponds to the straight line path connecting $0 \in \mathbb{R} \otimes_{\mathbb{Z}} P$ to $\nu \in P \subset$ $\mathbb{R} \otimes_{\mathbb{Z}} P$.

Remark 3.16. Let $\pi=\left(\nu_{1}, \nu_{2}, \ldots, \nu_{s} ; a_{0}, a_{1}, \ldots, a_{s}\right)$ be as above. If $a_{u}=u / s$ for all $0 \leq u \leq s$, then we write the corresponding path simply as $\pi=\left(\nu_{1}, \nu_{2}, \ldots, \nu_{s}\right)$. Now, noting that the set $\left\{\left\langle\lambda, \beta^{\vee}\right\rangle \mid \beta \in \Delta\right\} \subset \mathbb{Z}$ is a finite set (see (2.8)-(2.10) $)$, we define $N=N_{\lambda} \in \mathbb{Z}_{\geq 1}$ to be the least common multiple of all nonzero integers in $\left\{\left\langle\lambda, \beta^{\vee}\right\rangle \mid \beta \in \Delta\right\} \cup\{1\}$. It follows from the definition of $a$-chains that if $\pi=\left(\nu_{1}, \nu_{2}, \ldots, \nu_{s} ; a_{0}, a_{1}, \ldots, a_{s}\right) \in \mathbb{B}(\lambda)$, then $N a_{u} \in \mathbb{Z}$ for all $0 \leq u \leq s$. Consequently, the path corresponding to $\pi$ is identical to the path

$$
(\underbrace{\nu_{1}, \ldots, \nu_{1}}_{b_{1} \text { times }}, \underbrace{\nu_{2}, \ldots, \nu_{2}}_{b_{2} \text { times }}, \ldots, \underbrace{\nu_{s}, \ldots, \nu_{s}}_{b_{s} \text { times }}),
$$

where $b_{u}:=N\left(a_{u}-a_{u-1}\right)$ for $1 \leq u \leq s$.

We define a $U_{q}(\mathfrak{g})$-crystal structure on the set $\mathbb{B}(\lambda)$ of all LS paths of shape $\lambda$ as follows. First, recalling from [Li2 Lemma 4.5 a)] that $\pi(1) \in P$ for all $\pi \in \mathbb{B}(\lambda)$, we define wt $: \mathbb{B}(\lambda) \rightarrow P$ by wt $(\pi)=\pi(1)$.

Next, for $\pi \in \mathbb{B}(\lambda)$ and $i \in I$, we define $e_{i} \pi$ as follows. Set

$$
H_{i}^{\pi}(t):=\left\langle\pi(t), h_{i}\right\rangle \quad \text { for } t \in[0,1]_{\mathbb{R}}, \quad \text { and } \quad m_{i}^{\pi}:=\min \left\{H_{i}^{\pi}(t) \mid t \in[0,1]_{\mathbb{R}}\right\} .
$$

We know from $[$ Li2, Lemma $4.5 \mathrm{~d})]$ that $m_{i}^{\pi} \in \mathbb{Z}_{\leq 0}$. If $m_{i}^{\pi}=0$, then we set $e_{i} \pi:=\mathbf{0}$. If $m_{i}^{\pi} \leq-1$, then we set

$$
\left(e_{i} \pi\right)(t):= \begin{cases}\pi(t) & \text { if } 0 \leq t \leq t_{0} \\ \pi\left(t_{0}\right)+r_{i}\left(\pi(t)-\pi\left(t_{0}\right)\right) & \text { if } t_{0} \leq t \leq t_{1} \\ \pi(t)+\alpha_{i} & \text { if } t_{1} \leq t \leq 1\end{cases}
$$

where

$$
\begin{aligned}
t_{1} & :=\min \left\{t \in[0,1]_{\mathbb{R}} \mid H_{i}^{\pi}(t)=m_{i}^{\pi}\right\}, \\
t_{0} & :=\max \left\{t \in\left[0, t_{1}\right]_{\mathbb{R}} \mid H_{i}^{\pi}(t)=m_{i}^{\pi}+1\right\} .
\end{aligned}
$$

It follows from Li2, Corollary 2 in $\S 4]$ that $e_{i} \pi \in \mathbb{B}(\lambda) \cup\{\mathbf{0}\}$. Thus, we obtain a map $e_{i}: \mathbb{B}(\lambda) \rightarrow \mathbb{B}(\lambda) \cup\{\mathbf{0}\}$. Similarly, for $\pi \in \mathbb{B}(\lambda)$ and $i \in I$, we define $f_{i} \pi$ as 
follows. Note that $H_{i}^{\pi}(1)-m_{i}^{\pi} \in \mathbb{Z}_{\geq 0}$. If $H_{i}^{\pi}(1)-m_{i}^{\pi}=0$, then we set $f_{i} \pi:=\mathbf{0}$. If $H_{i}^{\pi}(1)-m_{i}^{\pi} \geq 1$, then we set

$$
\left(f_{i} \pi\right)(t):= \begin{cases}\pi(t) & \text { if } 0 \leq t \leq t_{0} \\ \pi\left(t_{0}\right)+r_{i}\left(\pi(t)-\pi\left(t_{0}\right)\right) & \text { if } t_{0} \leq t \leq t_{1} \\ \pi(t)-\alpha_{i} & \text { if } t_{1} \leq t \leq 1\end{cases}
$$

where

$$
\begin{aligned}
& t_{0}:=\max \left\{t \in[0,1]_{\mathbb{R}} \mid H_{i}^{\pi}(t)=m_{i}^{\pi}\right\}, \\
& t_{1}:=\min \left\{t \in\left[t_{0}, 1\right]_{\mathbb{R}} \mid H_{i}^{\pi}(t)=m_{i}^{\pi}+1\right\} .
\end{aligned}
$$

It follows from [Li2, Corollary 2 in $\S 4$ ] that $f_{i} \pi \in \mathbb{B}(\lambda) \cup\{\mathbf{0}\}$. Thus, we obtain a map $f_{i}: \mathbb{B}(\lambda) \rightarrow \mathbb{B}(\lambda) \cup\{\mathbf{0}\}$. Finally, for $\pi \in \mathbb{B}(\lambda)$ and $i \in I$, we set

$$
\varepsilon_{i}(\pi):=\max \left\{k \geq 0 \mid e_{i}^{k} \pi \neq \mathbf{0}\right\}, \quad \varphi_{i}(\pi):=\max \left\{k \geq 0 \mid f_{i}^{k} \pi \neq \mathbf{0}\right\} .
$$

Theorem 3.17 ([Li2, $\S 2$ and $\S 4])$. The set $\mathbb{B}(\lambda)$, equipped with the maps wt: $\mathbb{B}(\lambda) \rightarrow P, e_{i}, f_{i}: \mathbb{B}(\lambda) \rightarrow \mathbb{B}(\lambda) \cup\{\mathbf{0}\}$ for $i \in I$, and $\varepsilon_{i}, \varphi_{i}: \mathbb{B}(\lambda) \rightarrow \mathbb{Z}_{\geq 0}$ for $i \in I$, is a $U_{q}(\mathfrak{g})$-crystal.

Let $J$ be a finite interval in $I=\mathbb{Z}_{\geq 0}$. Recall from Lemma 3.15 that $\mathbb{B}_{J}(\lambda)$ is a subset of $\mathbb{B}(\lambda)$. We see from [Li2, Corollary 2 in $\S 4$ ] that if $\pi \in \mathbb{B}_{J}(\lambda) \subset \mathbb{B}(\lambda)$, then $e_{i} \pi, f_{i} \pi \in \mathbb{B}_{J}(\lambda) \cup\{\mathbf{0}\}$ for all $i \in J$. Consequently, the set $\mathbb{B}_{J}(\lambda)$, equipped with the restrictions to $\mathbb{B}_{J}(\lambda)$ of the maps wt $: \mathbb{B}(\lambda) \rightarrow P, e_{i}, f_{i}: \mathbb{B}(\lambda) \rightarrow \mathbb{B}(\lambda) \cup\{\mathbf{0}\}$ for $i \in J$, and $\varepsilon_{i}, \varphi_{i}: \mathbb{B}(\lambda) \rightarrow \mathbb{Z}_{\geq 0}$ for $i \in J$, is a $U_{q}\left(\mathfrak{g}_{J}\right)$-crystal.

Remark 3.18. It follows from Remark 3.14 $(2)$ and Lemma 3.15 that $\mathbb{B}_{J}(\lambda)=$ $\mathbb{B}_{J}(w \lambda)$ for all $w \in W_{J}$. Hence we have $\mathbb{B}_{J}(\lambda)=\mathbb{B}_{J}\left(\lambda_{J}\right)$. Also, we know from [J], Corollary 6.4.27] or [Kas4, Theorem 4.1] that $\mathbb{B}_{J}\left(\lambda_{J}\right)$ is isomorphic, as a $U_{q}\left(\mathfrak{g}_{J}\right)$ crystal, to the crystal basis of the finite-dimensional irreducible $U_{q}\left(\mathfrak{g}_{J}\right)$-module of highest weight $\lambda_{J}$. Combining these facts with Remark 3.5 yields the following isomorphism of $U_{q}\left(\mathfrak{g}_{J}\right)$-crystals:

$$
\mathcal{B}_{J}(\lambda) \cong \mathcal{B}_{J}\left(\lambda_{J}\right) \cong \mathbb{B}_{J}\left(\lambda_{J}\right)=\mathbb{B}_{J}(\lambda)
$$

In addition, the element $\pi_{\lambda_{J}}$ is contained in $\mathbb{B}_{J}(\lambda)=\mathbb{B}_{J}\left(\lambda_{J}\right)$ by Remark 3.14 (1) and Lemma 3.15. and it is a (unique) maximal element of weight $\lambda_{J}$ in $\mathbb{B}_{J}(\lambda)=\mathbb{B}_{J}\left(\lambda_{J}\right)$.

Proposition 3.19. Let $\lambda \in P$ be an integral weight.

(1) The crystal $\mathbb{B}(\lambda)$ is a normal $U_{q}(\mathfrak{g})$-crystal whose crystal graph is connected.

(2) The element $\pi_{\nu}=(\nu ; 0,1) \in \mathbb{B}(\lambda)$ is an extremal element for every $\nu \in W \lambda$.

Proof. (1) It follows from (3.7) and Remark 3.18 that $\mathbb{B}(\lambda)$ is a normal $U_{q}(\mathfrak{g})$-crystal. Also, using (3.7) and Remark 3.18, we deduce that the crystal graph of $\mathbb{B}(\lambda)$ is connected by the argument in the last paragraph of the proof of Proposition 3.6.

(2) We can easily show by induction on the length of $w \in W$ that $S_{w} \pi_{\nu}=\pi_{w \nu}$, from which the assertion follows immediately. This proves the proposition.

3.4. Isomorphism theorem. In this subsection, we prove that for an arbitrary $\lambda \in P$, the crystal $\mathbb{B}(\lambda)$ consisting of all LS paths of shape $\lambda$ is isomorphic to the crystal basis $\mathcal{B}(\lambda)$ of the extremal weight module $V(\lambda)$ of extremal weight $\lambda$ (see Corollary 3.21 (1) below). This gives a combinatorial realization in a unified way that is independent of the level $L_{\lambda}$ of $\lambda \in P$. Compare this with the related results 
of [Kw2, Theorem 3.5] (in type $A_{+\infty}$ ), [Kw3, Corollary 4.7 along with Remark 4.8] (in type $A_{\infty}$ ), and also [Le, §5] (in all types $A_{+\infty}, B_{\infty}, C_{\infty}, D_{\infty}$, but for $\lambda \in E$ ).

Recall from Proposition 3.19 (1) that $\mathbb{B}(\lambda)$ is a normal $U_{q}(\mathfrak{g})$-crystal for $\lambda \in P$. Also, note that the tensor product of normal $U_{q}(\mathfrak{g})$-crystals is again a normal $U_{q}(\mathfrak{g})$ crystal.

Theorem 3.20. Let $\lambda_{1}, \lambda_{2}, \ldots, \lambda_{a} \in P$ be integral weights, and set $\mathbb{B}:=\mathbb{B}\left(\lambda_{1}\right) \otimes$ $\mathbb{B}\left(\lambda_{2}\right) \otimes \cdots \otimes \mathbb{B}\left(\lambda_{a}\right)$. Suppose that $\pi:=\pi_{1} \otimes \pi_{2} \otimes \cdots \otimes \pi_{a} \in \mathbb{B}$ is an extremal element of weight $\nu \in P$, and denote by $\mathbb{B}(\pi)$ the connected component of $\mathbb{B}$ containing the extremal element $\pi$. Then, the crystal $\mathbb{B}(\pi)$ is isomorphic, as a $U_{q}(\mathfrak{g})$-crystal, to the crystal basis $\mathcal{B}(\nu)$ of the extremal weight $U_{q}(\mathfrak{g})$-module $V(\nu)$ of extremal weight $\nu$.

Proof. For $n \in \mathbb{Z}_{\geq 0}$, let $\mathbb{B}_{[n]}(\pi)$ denote the subset of $\mathbb{B}(\pi)$ consisting of all elements of the form $X \pi$ for some monomial $X$ in the Kashiwara operators $e_{i}$ and $f_{i}$ for $i \in[n]$. We claim that there exists an isomorphism $\Psi_{n}: \mathbb{B}_{[n]}(\pi) \stackrel{\sim}{\rightarrow} \mathcal{B}_{[n]}(\nu)$ of $U_{q}\left(\mathfrak{g}_{[n]}\right)$-crystals. Indeed, let $w \in W_{[n]}$ be such that $w \nu=\nu_{[n]}$. We deduce from the definitions of $S_{w}$ and $\mathbb{B}_{[n]}(\pi)$ that $S_{w} \pi$ is contained in $\mathbb{B}_{[n]}(\pi)$. In addition, since $\pi$ is extremal by assumption, it is $[n]$-extremal. Consequently, the element $S_{w} \pi \in \mathbb{B}_{[n]}(\pi)$ is an $[n]$-extremal element of weight $w \nu=\nu_{[n]}$. Furthermore, since $\nu_{[n]}$ is $[n]$-dominant by definition, we see from Definition [3.2 (1) that $S_{w} \pi$ is an $[n]$ maximal element of $\mathbb{B}_{[n]}(\pi)$. Also, because $\mathbb{B}$ is a normal $U_{q}(\mathfrak{g})$-crystal and because the crystal graph of the $U_{q}\left(\mathfrak{g}_{[n]}\right)$-crystal $\mathbb{B}_{[n]}(\pi)$ is connected, we find that $\mathbb{B}_{[n]}(\pi)$ is isomorphic, as a $U_{q}\left(\mathfrak{g}_{[n]}\right)$-crystal, to the crystal basis $\mathcal{B}_{[n]}\left(\nu_{[n]}\right)$, and hence to the crystal basis $\mathcal{B}_{[n]}(\nu)$ (see Remark 3.5).

In the sequel, we use the notation in the proof of Proposition 3.6. Recall that the crystal basis $\mathcal{B}_{[n]}(\nu)$ is isomorphic, as a $U_{q}\left(\mathfrak{g}_{[n]}\right)$-crystal, to $\mathcal{B}(\nu) \cap \mathcal{B}_{[n]}^{\nu}$. Hence we have a canonical embedding $\iota$ from $\mathcal{B}_{[n]}(\nu) \cong \mathcal{B}(\nu) \cap \mathcal{B}_{[n]}^{\nu}$ into $\mathcal{B}_{[n+1]}(\nu) \cong$ $\mathcal{B}(\nu) \cap \mathcal{B}_{[n+1]}^{\nu}($ see (3.5) $)$. Note that the embedding $\iota$ commutes with the Kashiwara operators $e_{i}$ and $f_{i}$ for $i \in[n]$. We show that the following diagram is commutative for all $n \in \mathbb{Z}_{\geq 0}$ :

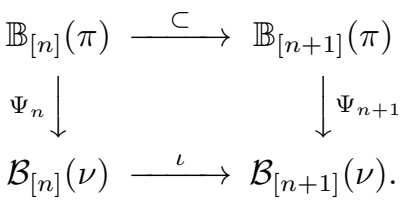

Let $\eta \in \mathbb{B}_{[n]}(\pi)$. Then, there exists a monomial $X$ in the Kashiwara operators $e_{i}$ and $f_{i}$ for $i \in[n]$ such that $\eta=X \pi$. It follows that $\iota\left(\Psi_{n}(\eta)\right)=\iota\left(\Psi_{n}(X \pi)\right)=$ $X \iota\left(\Psi_{n}(\pi)\right)$. Since $\pi$ is of weight $\nu$ by assumption, we deduce that $\iota\left(\Psi_{n}(\pi)\right)$ is the unique element of weight $\nu$ in $\mathcal{B}_{[n+1]}(\nu)$ (see Remark 3.5). Also, the element $\eta \in \mathbb{B}_{[n]}(\pi) \subset \mathbb{B}_{[n+1]}(\pi)$ is mapped to $\Psi_{n+1}(\eta)=\Psi_{n+1}(X \pi)=X \Psi_{n+1}(\pi)$ under the isomorphism $\Psi_{n+1}: \mathbb{B}_{[n+1]}(\pi) \stackrel{\sim}{\rightarrow} \mathcal{B}_{[n+1]}(\nu)$. By the same reasoning as above, we deduce that $\Psi_{n+1}(\pi)$ is the unique element of weight $\nu$ in $\mathcal{B}_{[n+1]}(\nu)$. This implies that $\iota\left(\Psi_{n}(\pi)\right)=\Psi_{n+1}(\pi)$. Therefore, we obtain $\iota\left(\Psi_{n}(\eta)\right)=\Psi_{n+1}(\eta)$, as desired.

The commutative diagram (3.10) allows us to define a map $\Psi: \mathbb{B}(\pi) \rightarrow \mathcal{B}(\nu)$ as follows: for $\eta \in \mathbb{B}(\pi)$,

$$
\Psi(\eta):=\Psi_{n}(\eta) \quad \text { if } \eta \in \mathbb{B}_{[n]}(\pi) \text { for some } n \in \mathbb{Z}_{\geq 0} .
$$


By using the definition, we can easily verify that the map $\Psi: \mathbb{B}(\pi) \rightarrow \mathcal{B}(\nu)$ is indeed an isomorphism of $U_{q}(\mathfrak{g})$-crystals. Thus we have proved the theorem.

Corollary 3.21. (1) Let $\lambda \in P$ be an integral weight. Then, the crystal $\mathbb{B}(\lambda)$ consisting of all LS paths of shape $\lambda$ is isomorphic, as a $U_{q}(\mathfrak{g})$-crystal, to the crystal basis $\mathcal{B}(\lambda)$ of the extremal weight $U_{q}(\mathfrak{g})$-module $V(\lambda)$ of extremal weight $\lambda$.

(2) For each $\lambda \in P$ and $w \in W$, we have $\mathbb{B}(\lambda)_{w \lambda}=\left\{S_{w} \pi_{\lambda}\right\}=\left\{\pi_{w \lambda}\right\}$.

(3) Let $\lambda, \mu \in P$. Then, $\mathbb{B}(\lambda) \cong \mathbb{B}(\mu)$ as $U_{q}(\mathfrak{g})$-crystals if and only if $\lambda \in W \mu$.

Proof. Part (1) follows immediately from Proposition 3.19 and Theorem 3.20, Parts (2) and (3) follow from Theorem 3.20, together with Propositions 3.7 and 3.9.

\section{Decomposition of tensor products into CONnECted COMPONEnts}

In this section, we consider the decomposition (into connected components) of the tensor product $\mathbb{B}(\lambda) \otimes \mathbb{B}(\mu)$ for $\lambda, \mu \in P$ with $L_{\lambda}, L_{\mu} \geq 0$. In fact, it turns out that each connected component is isomorphic to $\mathbb{B}(\nu)$ for some $\nu \in P$. Our main aim is to give an explicit description of the multiplicity $m_{\lambda, \mu}^{\nu}$ of a connected component $\mathbb{B}(\nu)$ for $\nu \in P$ in this decomposition. It should be mentioned that our results in this section can be regarded as extensions of the corresponding results in Kw2] (in type $A_{+\infty}$ ) and [Kw3] (in type $A_{\infty}$ ) to the cases of types $B_{\infty}, C_{\infty}, D_{\infty}$. See also [L] for the case $\lambda, \mu \in E$ (in all types $A_{+\infty}, B_{\infty}, C_{\infty}, D_{\infty}$ ).

4.1. The case $\lambda, \mu \in E$. A partition is, by definition, a weakly increasing sequence $\rho=\left(\rho^{(0)} \geq \rho^{(1)} \geq \rho^{(2)} \geq \cdots\right)$ of nonnegative integers such that $\rho^{(j)}=0$ for all but finitely many $j \in \mathbb{Z}_{\geq 0}$. We call $\rho^{(j)}$ the $j$-th part of $\rho$ for $j \in \mathbb{Z}_{\geq 0}$. Let $\mathcal{P}$ denote the set of all partitions. For $\rho=\left(\rho^{(0)} \geq \rho^{(1)} \geq \rho^{(2)} \geq \cdots\right) \in \mathcal{P}$, we define the length of $\rho$, denoted by $\ell(\rho)$, to be the number of nonzero parts of $\rho$ and define the size of $\rho$, denoted by $|\rho|$, to be the sum of all parts of $\rho$, i.e., $|\rho|:=\sum_{j \in \mathbb{Z}_{\geq 0}} \rho^{(j)}$. Also, for $\rho, \kappa, \omega \in \mathcal{P}$, let $\mathrm{LR}_{\rho, \kappa}^{\omega}$ denote the Littlewood-Richardson coefficient for $\rho$, $\kappa$, and $\omega$ (see, for example, [F, Chapter 5] or [Ko2, $\S 2]$ ). It is well-known that $\operatorname{LR}_{\rho, \kappa}^{\omega} \neq 0$ only if $|\omega|=|\rho|+|\kappa|$.

Now, let $P_{-}^{[1, \infty)}$ denote the subset of $E$ consisting of all elements $\nu$ such that the sequence $\left(\nu^{(0)}, \nu^{(1)}, \nu^{(2)}, \ldots\right)$ is a partition. Observe that if $\nu \in P_{-}^{[1, \infty)}$, then $\left\langle\nu, h_{i}\right\rangle \leq 0$ for all $i \in \mathbb{Z}_{\geq 1}$ (see (2.4) $)$, and hence $\nu$ is $[1, n]$-anti-dominant for all $n \in \mathbb{Z}_{\geq 1}$. We identify the set $P_{-}^{[1, \infty)}$ with the set $\mathcal{P}$ of all partitions via the correspondence $\nu \mapsto\left(\nu^{(0)}, \nu^{(1)}, \nu^{(2)}, \ldots\right)$. Under this correspondence, we have $|\nu|=\sum_{j \in \mathbb{Z}_{>0}}\left|\nu^{(j)}\right|$ and $\ell(\nu)=\# \operatorname{Supp}(\nu)=\max \operatorname{Supp}(\nu)+1$ for $\nu \in P_{-}^{[1, \infty)}$. Furthermore, using (2.5)-(2.7), we can easily verify that for each $\lambda \in E$, there exists a unique element $\lambda_{\dagger} \in W \lambda$ such that $\lambda_{\dagger} \in P_{-}^{[1, \infty)}(\cong \mathcal{P})$. In fact, the corresponding partition $\left(\lambda_{\dagger}^{(0)}, \lambda_{\dagger}^{(1)}, \lambda_{\dagger}^{(2)}, \ldots\right) \in \mathcal{P}$ is obtained by arranging the sequence $\left(\left|\lambda^{(0)}\right|,\left|\lambda^{(1)}\right|,\left|\lambda^{(2)}\right|, \ldots\right)$ of nonnegative integers in weakly increasing order. Thus, $P_{-}^{[1, \infty)}(\cong \mathcal{P})$ is a complete set of representatives for $W$-orbits in $E$. It follows from Corollary 3.21 (3) that if $\nu \neq \nu^{\prime}$ for $\nu, \nu^{\prime} \in P_{-}^{[1, \infty)}$, then $\mathbb{B}(\nu) ¥ \mathbb{B}\left(\nu^{\prime}\right)$ as $U_{q}(\mathfrak{g})$-crystals.

We can deduce the following theorem from [Le, Corollary 3.2.5 and Remark following Theorem 4.1.6] (see also [Kw2, Theorem 4.10] in type $A_{+\infty}$ and [Kw3. 
Proposition 4.9] in type $\left.A_{\infty}\right)$. In [NS, §4.1], another proof of this theorem is given by using LS paths.

Theorem 4.1. Let $\lambda, \mu \in E$. Then, we have the following decomposition into connected components:

$$
\mathbb{B}(\lambda) \otimes \mathbb{B}(\mu)=\bigoplus_{\nu \in P_{-}^{[1, \infty)}} \mathbb{B}(\nu)^{\oplus m_{\lambda, \mu}^{\nu}} \quad \text { as } U_{q}(\mathfrak{g}) \text {-crystals },
$$

where for each $\nu \in P_{-}^{[1, \infty)}(\cong \mathcal{P})$, the multiplicity $m_{\lambda, \mu}^{\nu}$ is equal to the LittlewoodRichardson coefficient $\mathrm{LR}_{\lambda_{+}, \mu_{+}}^{\nu}$ for the partitions $\lambda_{\dagger}, \mu_{\dagger}$, and $\nu$.

Remark 4.2. Because $\mathrm{LR}_{\lambda_{\dagger}, \mu_{\dagger}}^{\nu} \neq 0$ only if $|\nu|=\left|\lambda_{\dagger}\right|+\left|\mu_{\dagger}\right|$ as noted above, it follows that the total number of connected components of $\mathbb{B}(\lambda) \otimes \mathbb{B}(\mu)$ is finite for $\lambda, \mu \in E$.

4.2. The case $\lambda \in E, \mu \in P_{+}$. First we prove the following proposition (cf. Kw2, the proof of Corollary 4.11] in type $A_{+\infty}$ and [Kw3, Proposition 3.11] in type $A_{\infty}$ ).

Proposition 4.3. Let $\lambda \in E$ and $\mu \in P_{+}$. The crystal graph of the tensor product $\mathbb{B}(\lambda) \otimes \mathbb{B}(\mu)$ is connected.

Proof. Since $\mu \in P_{+}$, it follows from Corollary 3.21(1) and Remark 3.4(1) that $\mathbb{B}(\mu)$ is isomorphic, as a $U_{q}(\mathfrak{g})$-crystal, to the crystal basis of the irreducible highest weight $U_{q}(\mathfrak{g})$-module of highest weight $\mu$. Therefore, the weight wt $(\eta)$ of an element $\eta \in \mathbb{B}(\mu)$ is of the form $\operatorname{wt}(\eta)=\mu-\sum_{i \in I} m_{i} \alpha_{i}$ for some $m_{i} \in \mathbb{Z}_{\geq 0}, i \in I$. In this case, we set

$$
\operatorname{dep}(\eta):=\sum_{i \in I} m_{i} \in \mathbb{Z}_{\geq 0}
$$

Let $\mathbb{B}\left(\pi_{\lambda} \otimes \pi_{\mu}\right)$ denote the connected component of $\mathbb{B}(\lambda) \otimes \mathbb{B}(\mu)$ containing $\pi_{\lambda} \otimes \pi_{\mu}$. We will show by induction on $\operatorname{dep}(\eta)$ that every element $\pi \otimes \eta \in \mathbb{B}(\lambda) \otimes \mathbb{B}(\mu)$ is contained in this connected component $\mathbb{B}\left(\pi_{\lambda} \otimes \pi_{\mu}\right)$.

First, suppose that $\operatorname{dep}(\eta)=0$. Then we have $\eta=\pi_{\mu}$. Since the crystal graph of $\mathbb{B}(\lambda)$ is connected by Corollary $3.21(1)$, there exists a monomial $X_{1}$ in the Kashiwara operators $e_{i}$ and $f_{i}$ for $i \in I$ such that $X_{1} \pi=\pi_{\lambda}$. Note that if $f_{i} \pi^{\prime} \neq \mathbf{0}$ (resp., $e_{i} \pi^{\prime} \neq \mathbf{0}$ ) for some $\pi^{\prime} \in \mathbb{B}(\lambda)$ and $i \in I$, then $f_{i}\left(\pi^{\prime} \otimes \pi_{\mu}\right)=\left(f_{i} \pi^{\prime}\right) \otimes \pi_{\mu}$ (resp., $\left.e_{i}\left(\pi^{\prime} \otimes \pi_{\mu}\right)=\left(e_{i} \pi^{\prime}\right) \otimes \pi_{\mu}\right)$ by the tensor product rule for crystals. From this, it follows that $X_{1}\left(\pi \otimes \pi_{\mu}\right)=\left(X_{1} \pi\right) \otimes \pi_{\mu}=\pi_{\lambda} \otimes \pi_{\mu}$, as desired.

Next, suppose that $\operatorname{dep}(\eta)>0$. Note that $e_{i} \eta \neq \mathbf{0}$ for some $i \in I$ since $\eta$ is not a (unique) maximal element $\pi_{\mu}$ of $\mathbb{B}(\mu)$. Now we take $n \in \mathbb{Z}_{\geq 0}$ such that:

(i) $\operatorname{Supp}(\lambda) \varsubsetneqq[n]=\{0,1, \ldots, n\}$;

(ii) $\pi \in \mathbb{B}_{[n]}(\lambda)($ see (3.7));

(iii) $e_{i} \eta \neq \mathbf{0}$ for some $i \in[n]$.

It follows from condition (ii) and Remark 3.18 that there exists a monomial $X_{2}$ in $e_{i}^{\max }$ for $i \in[n]$ such that $X_{2} \pi=\pi_{\lambda_{[n]}}$. By the tensor product rule for crystals, we have $X_{2}(\pi \otimes \eta)=\left(X_{2} \pi\right) \otimes \eta^{\prime}=\pi_{\lambda_{[n]}} \otimes \eta^{\prime}$ for some $\eta^{\prime} \in \mathbb{B}(\mu)$. If $\eta^{\prime} \neq \eta$, then we have $\operatorname{dep}\left(\eta^{\prime}\right)<\operatorname{dep}(\eta)$ since $X_{2}$ is a monomial in $e_{i}^{\max }$ for $i \in[n]$. Therefore, by our induction hypothesis, $\pi_{\lambda_{[n]}} \otimes \eta^{\prime}$ is contained in the connected component $\mathbb{B}\left(\pi_{\lambda} \otimes \pi_{\mu}\right)$, and hence so is $\pi \otimes \eta$. Thus, we may assume that $\eta^{\prime}=\eta$, i.e.,

$$
X_{2}(\pi \otimes \eta)=\pi_{\lambda_{[n]}} \otimes \eta .
$$


We set $w_{i}:=r_{i+1} r_{i+2} \cdots r_{n} r_{n+1} \in W$ for $0 \leq i \leq n$, and $w_{n+1}:=e$, the identity element of $W$. From Lemma 2.3 and explicit computations using (2.1)-(2.3) and (2.5) -(2.7), we easily deduce the following claim.

Claim. We have $\left\langle w_{i} \lambda_{[n]}, h_{i}\right\rangle=\left\langle\lambda_{[n]}, w_{i}^{-1} h_{i}\right\rangle \leq 0$ for all $0 \leq i \leq n+1$.

Let $0 \leq i \leq n$. By the tensor product rule for crystals, we have

$$
\begin{aligned}
e_{i+1}^{\max } e_{i+2}^{\max } & \cdots e_{n}^{\max } e_{n+1}^{\max } X_{2}(\pi \otimes \eta) \\
& =e_{i+1}^{\max } e_{i+2}^{\max } \cdots e_{n}^{\max } e_{n+1}^{\max }\left(\pi_{\lambda_{[n]}} \otimes \eta\right) \quad \text { by (4.2) } \\
& =\left(e_{i+1}^{\max } e_{i+2}^{\max } \cdots e_{n}^{\max } e_{n+1}^{\max } \pi_{\lambda_{[n]}}\right) \otimes \eta_{i}
\end{aligned}
$$

for some $\eta_{i} \in \mathbb{B}(\mu)$. Using the claim above successively, we find that

$$
e_{i+1}^{\max } e_{i+2}^{\max } \cdots e_{n}^{\max } e_{n+1}^{\max } \pi_{\lambda_{[n]}}=\pi_{r_{i+1} r_{i+2} \cdots r_{n} r_{n+1} \lambda_{[n]}}=\pi_{w_{i} \lambda_{[n]}},
$$

and hence that

$$
e_{i+1}^{\max } e_{i+2}^{\max } \cdots e_{n}^{\max } e_{n+1}^{\max } X_{2}(\pi \otimes \eta)=\pi_{w_{i} \lambda_{[n]}} \otimes \eta_{i} .
$$

Let us take $i \in[n]$ such that $e_{i} \eta \neq \mathbf{0}$ (recall condition (iii)). If $\eta_{i} \neq \eta$, then we have $\operatorname{dep}\left(\eta_{i}\right)<\operatorname{dep}(\eta)$, and hence by our induction hypothesis, $\pi_{w_{i} \lambda_{[n]}} \otimes \eta_{i}$ is contained in $\mathbb{B}\left(\pi_{\lambda} \otimes \pi_{\mu}\right)$, which implies that $\pi \otimes \eta \in \mathbb{B}\left(\pi_{\lambda} \otimes \pi_{\mu}\right)$. Suppose, therefore, that $\eta_{i}=\eta$. Because $\left\langle w_{i} \lambda_{[n]}, h_{i}\right\rangle \leq 0$ by the claim above and because $e_{i} \eta \neq \mathbf{0}$ by the choice of $i \in[n]$, it follows from the tensor product rule for crystals that

$$
e_{i} e_{i+1}^{\max } e_{i+2}^{\max } \cdots e_{n}^{\max } e_{n+1}^{\max } X_{2}(\pi \otimes \eta)=e_{i}\left(\pi_{w_{i} \lambda_{[n]}} \otimes \eta\right)=\pi_{w_{i} \lambda_{[n]}} \otimes\left(e_{i} \eta\right) \quad(\neq \mathbf{0}) .
$$

Since $\operatorname{dep}\left(e_{i} \eta\right)<\operatorname{dep}(\eta)$, the element $\pi_{w_{i} \lambda_{[n]}} \otimes\left(e_{i} \eta\right)$ is contained in $\mathbb{B}\left(\pi_{\lambda} \otimes \pi_{\mu}\right)$ by our induction hypothesis. This implies that $\pi \otimes \eta \in \mathbb{B}\left(\pi_{\lambda} \otimes \pi_{\mu}\right)$, thereby completing the proof of Proposition 4.3 .

The following theorem extends [Kw2, Corollary 4.11] in type $A_{+\infty}$ and $\underline{\mathrm{Kw} 3}$, Theorem 4.6] in type $A_{\infty}$.

Theorem 4.4. Let $\lambda \in E$, and $\mu \in P_{+}$. Then

$$
\mathbb{B}(\lambda) \otimes \mathbb{B}(\mu) \cong \mathbb{B}(\xi+\mu) \quad \text { as } U_{q}(\mathfrak{g}) \text {-crystals }
$$

for some $\xi \in W \lambda$.

Proof. We set $q:=\min \left\{j \in \mathbb{Z}_{\geq 0} \mid \mu^{(j)}=L_{\mu}\right\}$. We see from Remark 2.1 that

$$
0 \leq \underbrace{\left\langle\mu^{(0)}\right\rangle \leq \mu^{(1)} \leq \cdots \leq \mu^{(q-1)}<}_{\text {If } q=0 \text {, then this part is omitted. }} L_{\mu}=\mu^{(q)}=\mu^{(q+1)}=\cdots .
$$

Also, we set $p:=\# \operatorname{Supp}(\lambda)$. Then we deduce by using (2.5) -(2.7) that there exists a unique element $\xi \in W \lambda$ satisfying the conditions that $\operatorname{Supp}(\xi)=[q, q+p-1]$ and that

$$
0<\xi^{(q)} \leq \xi^{(q+1)} \leq \cdots \leq \xi^{(q+p-1)} .
$$

Here we note that $\pi_{\xi} \in \mathbb{B}(\lambda)$ (see Remark 3.14(1), (2)). We will prove that $\pi_{\xi} \otimes$ $\pi_{\mu} \in \mathbb{B}(\lambda) \otimes \mathbb{B}(\mu)$ is an extremal element. If we prove this assertion, then it follows immediately from Theorem 3.20. Corollary 3.21(1), and Proposition 4.3 that $\mathbb{B}(\lambda) \otimes \mathbb{B}(\mu) \cong \mathbb{B}(\xi+\mu)$ as $U_{q}(\mathfrak{g})$-crystals, which is what we want to prove.

The following claim is easily shown by using (2.8)-(2.10). 
Claim. Let $\beta \in \Delta$ be a root for $\mathfrak{g}$.

(1) If $\left\langle\xi, \beta^{\vee}\right\rangle>0$, then $\left\langle\mu, \beta^{\vee}\right\rangle \geq 0$.

(2) If $\left\langle\mu, \beta^{\vee}\right\rangle>0$, then $\left\langle\xi, \beta^{\vee}\right\rangle \geq 0$.

By an argument entirely similar to the one for [AK, Lemma 1.6 (1)], we deduce, using the claim above, that $S_{w}\left(\pi_{\xi} \otimes \pi_{\mu}\right)=\pi_{w \xi} \otimes \pi_{w \mu}$ for all $w \in W$ (the proof proceeds by induction on the length of $w \in W$ with the help of Corollary 3.21(2)). From this, it easily follows that $\pi_{\xi} \otimes \pi_{\mu}$ is extremal. This completes the proof of Theorem 4.4 .

Remark 4.5. Let $\lambda \in P$ be an integral weight such that $L_{\lambda} \geq 0$. We set

$$
q:=\#\left\{j \in \mathbb{Z}_{\geq 0}|| \lambda^{(j)} \mid<L_{\lambda}\right\} \quad \text { and } \quad p:=\#\left\{j \in \mathbb{Z}_{\geq 0}|| \lambda^{(j)} \mid>L_{\lambda}\right\} .
$$

Then, we deduce by using (2.5)-(2.7) that there exists a unique element $\nu \in W \lambda$ satisfying the conditions that

$$
\left\{\begin{array}{l}
0 \leq \underbrace{\left\langle\nu^{(0)}\right\rangle \leq \nu^{(1)} \leq \cdots \leq \nu^{(q-1)}<}_{\text {If } q=0, \text { then this part is omitted. }} L_{\lambda} \leq \nu_{\text {If } p=0, \text { then this part is omitted. }}^{\nu^{(q)} \leq \nu^{(q+1)} \leq \cdots \leq \nu^{(q+p-1)}} \\
\nu^{(j)}=L_{\lambda} \text { for all } j \geq p+q .
\end{array}\right.
$$

We set

$$
\lambda^{+}:=\sum_{j=0}^{q-1} \nu^{(j)} \mathbf{e}_{j}+L_{\lambda}\left(\mathbf{e}_{q}+\mathbf{e}_{q+1}+\cdots\right), \quad \lambda^{0}:=\sum_{j=q}^{q+p-1}\left(\nu^{(j)}-L_{\lambda}\right) \mathbf{e}_{j} .
$$

Note that $\lambda^{+} \in P_{+}, \lambda^{0} \in E$, and $\nu=\lambda^{+}+\lambda^{0}$. Theorem 4.4 along with its proof, yields the isomorphism of $U_{q}(\mathfrak{g})$-crystals:

$$
\mathbb{B}(\lambda)=\mathbb{B}(\nu) \cong \mathbb{B}\left(\lambda^{0}\right) \otimes \mathbb{B}\left(\lambda^{+}\right) .
$$

The following proposition will be used in the proof of Theorem 4.17 below (cf. [Kw2, Lemma 5.1] in type $A_{+\infty}$ and [Kw3, Proposition 3.12] in type $A_{\infty}$ ).

Proposition 4.6. Let $\lambda_{1}, \lambda_{2} \in E$, and $\mu_{1}, \mu_{2} \in P_{+}$. Then

$$
\mathbb{B}\left(\lambda_{1}\right) \otimes \mathbb{B}\left(\mu_{1}\right) \cong \mathbb{B}\left(\lambda_{2}\right) \otimes \mathbb{B}\left(\mu_{2}\right) \quad \text { as } U_{q}(\mathfrak{g}) \text {-crystals }
$$

if and only if $\lambda_{1} \in W \lambda_{2}$ and $\mu_{1}=\mu_{2}$.

Proof. The "if" part follows immediately from Corollary 3.21(3). We show the "only if" part. As in the proof of Theorem 4.4, we set $q_{m}:=\min \left\{j \in \mathbb{Z}_{\geq 0} \mid \mu_{m}^{(j)}=\right.$ $\left.L_{\mu_{m}}\right\}$ and $p_{m}:=\# \operatorname{Supp}\left(\lambda_{m}\right)$ for $m=1$, 2. Also, for $m=1,2$, let $\xi_{m} \in W \lambda_{m}$ be the unique element of $W \lambda_{m}$ such that $0<\xi_{m}^{\left(q_{m}\right)} \leq \xi_{m}^{\left(q_{m}+1\right)} \leq \cdots \leq \xi_{m}^{\left(q_{m}+p_{m}-1\right)}$. Then, the proof of Theorem 4.4 shows that

$$
\mathbb{B}\left(\lambda_{m}\right) \otimes \mathbb{B}\left(\mu_{m}\right) \cong \mathbb{B}\left(\xi_{m}+\mu_{m}\right) \text { as } U_{q}(\mathfrak{g}) \text {-crystals }
$$

for $m=1,2$. Observe that if we set $\nu_{m}:=\xi_{m}+\mu_{m}$ for $m=1,2$, then we have $(4.5)$

$$
\left\{\begin{array}{l}
0 \leq \underbrace{\left\langle\nu_{m}^{(0)}\right\rangle \leq \nu_{m}^{(1)} \leq \cdots \leq \nu_{m}^{\left(q_{m}-1\right)}<}_{\text {If } q_{m}=0, \text { then this part is omitted. }} L_{\mu_{m}} \underbrace{\left\langle\nu_{m}^{\left(q_{m}\right)} \leq \nu_{m}^{\left(q_{m}+1\right)} \leq \cdots \leq \nu_{m}^{\left(q_{m}+p_{m}-1\right)}\right.}_{\text {If } p_{m}=0, \text { then this part is omitted. }}, \\
\nu_{m}^{(j)}=L_{\mu_{m}} \text { for all } j \geq p_{m}+q_{m} .
\end{array}\right.
$$


Because

$$
\mathbb{B}\left(\nu_{1}\right) \cong \mathbb{B}\left(\lambda_{1}\right) \otimes \mathbb{B}\left(\mu_{1}\right) \cong \mathbb{B}\left(\lambda_{2}\right) \otimes \mathbb{B}\left(\mu_{2}\right) \cong \mathbb{B}\left(\nu_{2}\right) \text { as } U_{q}(\mathfrak{g}) \text {-crystals }
$$

by assumption, we infer by Corollary $3.21(3)$ that $\nu_{1} \in W \nu_{2}$. However, by using (2.5) -(2.7), we see that $\nu_{2}$ is a unique element of $W \nu_{2}$ satisfying condition (4.5), and hence that $\nu_{1}=\nu_{2}$. From this equality, it is easily seen that $L_{\mu_{1}}=L_{\mu_{2}}$, and $q_{1}=q_{2}, p_{1}=p_{2}$. Therefore, it follows from the definitions of $\nu_{1}$ and $\nu_{2}$ that $\mu_{1}=\mu_{2}$ and hence that $\xi_{1}=\xi_{2}$. This proves the proposition.

4.3. The case $\lambda \in P_{+}, \mu \in E$. Throughout this subsection, we fix $\lambda \in P_{+}$and $\mu \in E$. We set $p:=\min \left\{j \in \mathbb{Z}_{\geq 0} \mid \lambda^{(j)}=L_{\lambda}\right\} \in \mathbb{Z}_{\geq 0}$. It follows from Remark 2.1 that

$$
0 \leq \underbrace{\left\langle\lambda^{(0)}\right\rangle \leq \lambda^{(1)} \leq \cdots \leq \lambda^{(p-1)}<}_{\text {If } p=0, \text { then this part is omitted. }} L_{\lambda}=\lambda^{(p)}=\lambda^{(p+1)}=\cdots .
$$

Also, we set

$$
q:=\min \left\{q \in \mathbb{Z}_{\geq 1} \mid \operatorname{Supp}(\mu) \subset[q-1] \text { and } q \geq|\mu|\right\},
$$

where $|\mu|:=\sum_{j \in \mathbb{Z}_{>0}}\left|\mu^{(j)}\right| \in \mathbb{Z}_{\geq 0}$. Here we remark that $q \geq \# \operatorname{Supp}(\mu)$. Recall from Remark [3.16] that if $N=N_{\mu} \in \mathbb{Z}_{\geq 1}$ denotes the least common multiple of nonzero integers in $\left\{\left\langle\mu, \beta^{\vee}\right\rangle \mid \beta \in \Delta\right\} \cup\{1\}$, then each LS path $\eta \in \mathbb{B}(\mu)$ of shape $\mu$ can be written as $\eta=\left(\nu_{1}, \nu_{2}, \ldots, \nu_{N}\right)$ for some $\nu_{1}, \nu_{2}, \ldots, \nu_{N} \in W \mu$, with $\nu_{1} \geq \nu_{2} \geq \cdots \geq \nu_{N}$.

Let $n \in \mathbb{Z}_{\geq 0}$ be such that $n>p+(N+1) q$. Note that $n \geq 3$. Let $P_{+}^{[n]}(\lambda, \mu)$ denote the subset of $P$ consisting of all $[n]$-dominant integral weights $\nu \in P$ satisfying the conditions that

$$
\left\{\begin{array}{l}
L_{\nu}=L_{\lambda}, \\
\nu^{(j)}=L_{\nu}\left(=L_{\lambda}\right) \quad \text { for all } j \geq n+1, \text { and } \\
\#\left\{0 \leq j \leq n \mid \nu^{(j)}=L_{\nu}\left(=L_{\lambda}\right)\right\}>n-p-N q .
\end{array}\right.
$$

Note that $n-p-N q \geq q \geq|\mu|$.

Lemma 4.7. Let $n \in \mathbb{Z}_{\geq 0}$ be such that $n>p+(N+1) q$. If $\pi \otimes \eta \in \mathbb{B}_{[n]}(\lambda) \otimes \mathbb{B}_{[n]}(\mu)$ is an $[n]$-maximal element, then $\pi=\pi_{\lambda}$. Moreover, the weight wt $(\pi \otimes \eta)$ of $\pi \otimes \eta$ is contained in $P_{+}^{[n]}(\lambda, \mu)$.

Proof. For the first assertion, observe that if $e_{i} \pi \neq \mathbf{0}$ for some $i \in[n]$, then $e_{i}(\pi \otimes \eta) \neq \mathbf{0}$ by the tensor product rule for crystals. Now, since $\pi \otimes \eta$ is $[n]$-maximal by assumption, we have $e_{i} \pi=\mathbf{0}$ for all $i \in[n]$, which implies that $\pi=\pi_{\lambda_{[n]}}=\pi_{\lambda}$ (see Remark 3.18), as desired.

We show that $\nu:=\operatorname{wt}(\pi \otimes \eta)=\operatorname{wt}\left(\pi_{\lambda} \otimes \eta\right)=\lambda+\operatorname{wt}(\eta)$ is contained in the set $P_{+}^{[n]}(\lambda, \mu)$. Since $\pi \otimes \eta$ is $[n]$-maximal and $\mathbb{B}(\lambda) \otimes \mathbb{B}(\mu)$ is a normal $U_{q}(\mathfrak{g})$ crystal, it is clear that $\nu=\operatorname{wt}(\pi \otimes \eta)$ is $[n]$-dominant. Also, we see that the level $L_{\nu}$ of $\nu=\lambda+\operatorname{wt}(\eta)$ is equal to $L_{\lambda}+0=L_{\lambda}$. We write $\eta \in \mathbb{B}_{[n]}(\mu)$ as $\eta=\left(\nu_{1}, \nu_{2}, \ldots, \nu_{N}\right)$ for some $\nu_{1}, \nu_{2}, \ldots, \nu_{N} \in W \mu$. Note that $\nu_{M} \in W_{[n]} \mu$ for all $1 \leq M \leq N$ by Lemma 3.15. Since $\operatorname{Supp}(\mu) \subset[q-1] \subset[n]$ by our assumptions, we have $\operatorname{Supp}\left(\nu_{M}\right) \subset[n]$ for all $1 \leq M \leq N$. Therefore, it follows from the equation $\nu=\lambda+\operatorname{wt}(\eta)=\lambda+(1 / N) \sum_{M=1}^{N} \nu_{M}$ that $\nu^{(j)}=L_{\lambda}$ for all $j \geq n+1$. 
Furthermore, since $\# \operatorname{Supp}\left(\nu_{M}\right)=\# \operatorname{Supp}(\mu) \leq q$ for all $1 \leq M \leq N$ and since $\operatorname{Supp}(\operatorname{wt}(\eta)) \subset \bigcup_{M=1}^{N} \operatorname{Supp}\left(\nu_{M}\right)$, we have

$$
\begin{aligned}
\#\{0 & \left.\leq j \leq n \mid \nu^{(j)}=L_{\nu}\left(=L_{\lambda}\right)\right\} \geq \#\left\{p \leq j \leq n \mid \nu^{(j)}=L_{\lambda}\right\} \\
& \geq(n-p+1)-\# \operatorname{Supp}(\operatorname{wt}(\eta)) \geq(n-p+1)-\sum_{M=1}^{N} \# \operatorname{Supp}\left(\nu_{M}\right) \\
& \geq(n-p+1)-N q>n-p-N q .
\end{aligned}
$$

Thus, we have shown that $\nu=\operatorname{wt}(\pi \otimes \eta) \in P_{+}^{[n]}(\lambda, \mu)$, thereby proving the lemma.

Let $n \in \mathbb{Z}_{\geq 0}$ be such that $n>p+(N+1) q$. We deduce from the definition of $P_{+}^{[n]}(\lambda, \mu)$ that an element $\nu \in P_{+}^{[n]}(\lambda, \mu)$ satisfies the conditions that

$$
\left\{\begin{array}{l}
0 \leq\left\langle\nu^{(0)}\right\rangle \leq \nu^{(1)} \leq \cdots \leq \nu^{\left(u_{0}-1\right)}<L_{\lambda} \\
\nu^{\left(u_{0}\right)}=\nu^{\left(u_{0}+1\right)}=\cdots=\nu^{\left(u_{1}-1\right)}=L_{\lambda} \\
L_{\lambda}<\nu^{\left(u_{1}\right)} \leq \nu^{\left(u_{1}+1\right)} \leq \cdots \leq \nu^{(n)} \\
\nu^{(j)}=L_{\lambda} \text { for all } j \geq n+1
\end{array}\right.
$$

for some $0 \leq u_{0}<u_{1} \leq n+1$. It follows from the definition of $P_{+}^{[n]}(\lambda, \mu)$ that $u_{1}-u_{0}>n-p-N q>q>0$. Here, if $u_{0}=0$ (resp., $u_{1}=n+1$ ), then the first condition (resp., the third condition) is dropped. For the element $\nu \in P_{+}^{[n]}(\lambda, \mu)$ above, we define $\xi \in P$ by

$$
\left\{\begin{array}{l}
\xi^{(j)}=\nu^{(j)} \quad \text { for } 0 \leq j \leq u_{0}-1, \\
\xi^{(j)}=\nu^{(j)}=L_{\lambda} \quad \text { for } u_{0} \leq j \leq u_{1}-1, \\
\xi^{\left(u_{1}\right)}=L_{\lambda}, \\
\xi^{(j)}=\nu^{(j-1)} \quad \text { for } u_{1}+1 \leq j \leq n+1, \\
\xi^{(j)}=\nu^{(j)}=L_{\lambda} \quad \text { for } j \geq n+2,
\end{array}\right.
$$

where $u_{0}$ and $u_{1}$ are as in (4.7). It is easily verified that $\xi \in P_{+}^{[n+1]}(\lambda, \mu)$.

Lemma 4.8. Keep the setting above. We have $\xi=\nu_{[n+1]}$. Moreover, if we define a map $\Theta_{n}: P_{+}^{[n]}(\lambda, \mu) \rightarrow P_{+}^{[n+1]}(\lambda, \mu)$ by $\Theta_{n}(\nu):=\nu_{[n+1]}$ for $\nu \in P_{+}^{[n]}(\lambda, \mu)$, then the map $\Theta_{n}$ is bijective.

Proof. We set $w:=r_{u_{1}+1} r_{u_{1}+2} \cdots r_{n} r_{n+1} \in W_{[n+1]}$, where $u_{1}$ is as in (4.7). Then, by using (2.5)-(2.7), we find that the $\xi$ given by (4.8) is identical to the element $w \nu \in W_{[n+1]} \nu$. Since $\xi$ is $[n+1]$-dominant by the definition (4.8) of $\xi$, we conclude that $\xi=\nu_{[n+1]}$. Thus we have shown that if $\nu \in P_{+}^{[n]}(\lambda, \mu)$, then $\Theta_{n}(\nu)=\nu_{[n+1]} \in$ $P_{+}^{[n+1]}(\lambda, \mu)$. Now, the bijectivity of the map $\Theta_{n}$ easily follows by examining (4.7) and (4.8). This proves the lemma.

Let $n \in \mathbb{Z}_{\geq 0}$ be such that $n>p+(N+1) q$. Let $\pi \otimes \eta$ be an $[n+1]$-maximal element of $\mathbb{B}_{[n+1]}(\lambda) \otimes \mathbb{B}_{[n+1]}(\mu)$. Then, we have $\operatorname{wt}(\pi \otimes \eta) \in P_{+}^{[n+1]}(\lambda, \mu)$ by Lemma 4.7 and hence $\operatorname{wt}(\pi \otimes \eta)=\nu_{[n+1]}$ for some $\nu \in P_{+}^{[n]}(\lambda, \mu)$ by Lemma 4.8. 
Proposition 4.9. Keep the setting above. Let $x \in W_{[n+1]}$ be such that $x \nu_{[n+1]}=\nu$. Then, $S_{x}(\pi \otimes \eta)$ is an $[n]$-maximal element of weight $\nu$ contained in $\mathbb{B}_{[n]}(\lambda) \otimes \mathbb{B}_{[n]}(\mu)$.

Proof. We will prove that there exists $w \in W_{[n+1]}$ such that $S_{w}(\pi \otimes \eta)$ is an $[n]$ maximal element contained in $\mathbb{B}_{[n]}(\lambda) \otimes \mathbb{B}_{[n]}(\mu)$. First of all, we see by Lemma 4.7 that $\pi=\pi_{\lambda}$. We write $\eta \in \mathbb{B}_{[n+1]}(\mu)$ as $\eta=\left(\nu_{1}, \nu_{2}, \ldots, \nu_{N}\right)$ for some $\nu_{1}, \nu_{2}, \ldots$, $\nu_{N} \in W \mu$, with $\nu_{1} \geq \nu_{2} \geq \cdots \geq \nu_{N}$. By the same reasoning as in the proof of Lemma 4.7 we deduce that $\nu_{M} \in W_{[n+1]} \mu$ and that $\operatorname{Supp}\left(\nu_{M}\right) \subset[n+1]$ for all $1 \leq M \leq N$. Furthermore, since $\left\langle\lambda, h_{i}\right\rangle=0$ for all $i \geq p+1$ by (4.6) and since $\pi \otimes \eta=\pi_{\lambda} \otimes \eta$ is $[n+1]$-maximal by assumption, it follows from the tensor product rule for crystals that $e_{i} \eta=\mathbf{0}$ for all $p+1 \leq i \leq n+1$. Consequently, by the definition of the Kashiwara operators $e_{i}$ for $p+1 \leq i \leq n+1$, we must have $m_{i}^{\eta}=\min \left\{\left\langle\eta(t), h_{i}\right\rangle \mid t \in[0,1]_{\mathbb{R}}\right\}=0$ for all $p+1 \leq i \leq n+1$. Therefore, it follows that $\left\langle\nu_{1}, h_{i}\right\rangle \geq 0$ for all $p+1 \leq i \leq n+1$. From this fact, using (2.4), we deduce that

$$
\left\{\begin{array}{l}
\nu_{1}^{(p)} \leq \nu_{1}^{(p+1)} \leq \cdots \leq \nu_{1}^{\left(s_{0}\right)}<0, \\
\nu_{1}^{\left(s_{0}+1\right)}=\nu_{1}^{\left(s_{0}+2\right)}=\cdots=\nu_{1}^{\left(s_{1}\right)}=0, \\
0<\nu_{1}^{\left(s_{1}+1\right)} \leq \nu_{1}^{\left(s_{1}+2\right)} \leq \cdots \leq \nu_{1}^{(n+1)}
\end{array}\right.
$$

for some $p-1 \leq s_{0} \leq s_{1} \leq n+1$. Here, since $\# \operatorname{Supp}\left(\nu_{1}\right)=\# \operatorname{Supp}(\mu) \leq q$, the number $\left(s_{0}-p+1\right)+\left(n-s_{1}+1\right)$ of nonzero elements in (4.9) is less than or equal to $q$, and hence we have

$$
\begin{aligned}
s_{1}-s_{0} & =(n-p+2)-\left\{\left(s_{0}-p+1\right)+\left(n-s_{1}+1\right)\right\} \\
& >(n-p+2)-q \geq p+(N+1) q-p+2-q=N q+2 .
\end{aligned}
$$

Also, since $\operatorname{wt}(\eta)=(1 / N) \sum_{M=1}^{N} \nu_{M}$, we obtain

$$
\operatorname{Supp}(\operatorname{wt} \eta) \subset \bigcup_{M=1}^{N} \operatorname{Supp}\left(\nu_{M}\right) \subset[n+1],
$$

and hence

$$
\# \operatorname{Supp}(\operatorname{wt} \eta) \leq \sum_{M=1}^{N} \# \operatorname{Supp}\left(\nu_{M}\right)=\sum_{M=1}^{N} \# \operatorname{Supp}(\mu) \leq \sum_{M=1}^{N} q=N q .
$$

From this inequality and (4.10), we infer that there exists $s_{0}+1<s \leq s_{1}$ such that $s \notin \operatorname{Supp}(\operatorname{wt}(\eta))$. We set $w_{1}:=r_{n+1} r_{n} \cdots r_{s+2} r_{s+1} \in W_{[n+1]}$ and $\eta_{1}:=S_{w_{1}} \eta \in$ $\mathbb{B}_{[n+1]}(\mu)$. Here we note that $s+1>s_{0}+2 \geq p+1$. Since $\left\langle\lambda, h_{i}\right\rangle=0$ for all $i \geq p+1$, by the tensor product rule for crystals, there follows

$$
S_{w_{1}}(\pi \otimes \eta)=S_{w_{1}}\left(\pi_{\lambda} \otimes \eta\right)=\pi_{\lambda} \otimes\left(S_{w_{1}} \eta\right)=\pi_{\lambda} \otimes \eta_{1} .
$$

We claim that $\eta_{1} \in \mathbb{B}_{[n]}(\mu)$. Write it as $\eta_{1}=\left(\xi_{1}, \xi_{2}, \ldots, \xi_{N}\right)$ for some $\xi_{1}, \xi_{2}, \ldots$, $\xi_{N} \in W \mu$, with $\xi_{1} \geq \xi_{2} \geq \cdots \geq \xi_{N}$. Then we have $\xi_{M}^{(n+1)} \geq \xi_{1}^{(n+1)} \geq 0$ for all $1 \leq M \leq N$. Indeed, it follows from the definitions of the Kashiwara operators $e_{i}$ and $f_{i}$ for $i \in[n+1]$ (see also [Li2, Proposition 4.7]) that $\xi_{1}=z \nu_{1}$ for some $z \in W_{[n+1]}$ obtained from $w_{1}=r_{n+1} r_{n} \cdots r_{s+2} r_{s+1}$ by taking a subexpression. Since $s_{0}+1<s \leq s_{1}$, we infer from (4.9), by using (2.5)-(2.7), that $\xi_{1}^{(n+1)} \geq$ 0 . Now, by the same reasoning as in the proof of Lemma 4.7 we deduce that $\operatorname{Supp}\left(\xi_{M}\right) \subset[n+1]$ for all $1 \leq M \leq N$ and hence that $\operatorname{Supp}\left(\xi_{M}-\xi_{1}\right) \subset[n+1]$. Also, 
since $\xi_{1} \geq \xi_{M}$, we have $\xi_{M}-\xi_{1} \in \sum_{i \in I} \mathbb{Z}_{\geq 0} \alpha_{i} \subset E$ by Remark 3.11 Combining these facts, we see that $\xi_{M}-\xi_{1} \in \sum_{i=0}^{n+1} \mathbb{Z}_{\geq 0} \alpha_{i}$ since $\alpha_{i}=-\mathbf{e}_{i-1}+\mathbf{e}_{i}$ for $i \geq 1$ (see (2.4)). Observe that by (2.4), $\xi \in \sum_{i=0}^{n+1} \mathbb{Z}_{\geq 0} \alpha_{i}$ implies that $\xi^{(n+1)} \geq 0$ since $\alpha_{n+1}^{(n+1)}=1>0$ and $\alpha_{i}^{(n+1)}=0$ for all $0 \leq i \leq n$. Therefore, $\left(\xi_{M}-\xi_{1}\right)^{(n+1)} \geq 0$ and hence $\xi_{M}^{(n+1)} \geq \xi_{1}^{(n+1)} \geq 0$, as desired. Furthermore, since $s \notin \operatorname{Supp}(\mathrm{wt} \eta$ ) and since $\operatorname{Supp}(\operatorname{wt}(\eta)) \subset[n+1]$ by (4.11), we can easily verify by using (2.5)(2.7) that $\operatorname{Supp}\left(\operatorname{wt}\left(\eta_{1}\right)\right)=\operatorname{Supp}\left(w_{1}(\operatorname{wt}(\eta))\right) \subset[n]$. It follows from the equation $\operatorname{wt}\left(\eta_{1}\right)=(1 / N) \sum_{M=1}^{N} \xi_{M}$ that $\xi_{M}^{(n+1)}=0$ for all $1 \leq M \leq N$, and hence that $\operatorname{Supp}\left(\xi_{M}\right) \subset[n]$ for all $1 \leq M \leq N$. In addition, since $\xi_{M} \in W \mu$ for $1 \leq M \leq N$, we see through use of (2.5) -(2.7) that $\# \operatorname{Supp}\left(\xi_{M}\right)=\# \operatorname{Supp}(\mu) \leq q<n$, and hence that $\operatorname{Supp}\left(\xi_{M}\right) \varsubsetneqq[n]$ for all $1 \leq M \leq N$. Recall that $\operatorname{Supp}(\mu) \subset[q-1] \varsubsetneqq[n]$ by the definition of $q$. Hence we can apply Lemma 2.3 to $\xi_{M}$ for $1 \leq M \leq N$, and also to $\mu$. Then, noting that $\xi_{M} \in W \mu$ for $1 \leq M \leq N$, we find through use of (2.5) -(2.7) that the unique [n]-dominant element of $W_{[n]} \xi_{M}$ is identical to the unique [n]-dominant element of $W_{[n]} \mu$ for all $1 \leq M \leq N$. As a consequence, we conclude that $\xi_{M} \in W_{[n]} \mu$ for all $1 \leq M \leq N$, which implies that $\eta_{1} \in \mathbb{B}_{[n]}(\mu)$ by Lemma 3.15. Thus we have shown that $S_{w_{1}}(\pi \otimes \eta)=\pi_{\lambda} \otimes \eta_{1} \in \mathbb{B}_{[n]}(\lambda) \otimes \mathbb{B}_{[n]}(\mu)$.

Because $\mathbb{B}(\lambda) \otimes \mathbb{B}(\mu)$ is a normal $U_{q}(\mathfrak{g})$-crystal and because $\pi \otimes \eta=\pi_{\lambda} \otimes \eta$ is $[n+1]$-maximal by assumption, it follows that $\pi \otimes \eta=\pi_{\lambda} \otimes \eta$ is $[n+1]$-extremal, and hence that so is $\pi_{\lambda} \otimes \eta_{1}=S_{w_{1}}(\pi \otimes \eta)$ (recall that $\left.w_{1} \in W_{[n+1]}\right)$. If we take $w_{2} \in W_{[n]}$ such that $w_{2}\left(\operatorname{wt}\left(\pi_{\lambda} \otimes \eta_{1}\right)\right)$ is $[n]$-dominant, then we see from (3.2) that the element $S_{w_{2}}\left(\pi_{\lambda} \otimes \eta_{1}\right) \in \mathbb{B}_{[n]}(\lambda) \otimes \mathbb{B}_{[n]}(\mu)$ is $[n]$-maximal since it is [n]-extremal. Now we set $w=w_{2} w_{1} \in W_{[n+1]}$. Then the element $S_{w}(\pi \otimes \eta)=S_{w_{2}}\left(\pi_{\lambda} \otimes \eta_{1}\right)$ is an $[n]$-maximal element contained in $\mathbb{B}_{[n]}(\lambda) \otimes \mathbb{B}_{[n]}(\mu)$. This proves the assertion at the very beginning of our proof.

It remains to show that $S_{w}(\pi \otimes \eta)$ is identical to $S_{x}(\pi \otimes \eta)$. If we set $\xi:=$ $\operatorname{wt}\left(S_{w}(\pi \otimes \eta)\right)$, then $\xi \in P_{+}^{[n]}(\lambda, \mu)$ by Lemma 4.7 Since $\nu_{[n+1]}=\operatorname{wt}(\pi \otimes \eta)=$ $w^{-1} \xi \in W_{[n+1]} \xi$, we have $\xi_{[n+1]}=\nu_{[n+1]}$. Therefore, by the bijectivity of the map $\Theta_{n}: P_{+}^{[n]}(\lambda, \mu) \rightarrow P_{+}^{[n+1]}(\lambda, \mu)$ (see Lemma 4.8), we obtain $\xi=\nu$, and hence $w \nu_{[n+1]}=\nu$. Since $\nu_{[n+1]}$ is $[n+1]$-dominant and since $x, w$ are elements of $W_{[n+1]}$ such that $w \nu_{[n+1]}=\nu=x \nu_{[n+1]}$, there exists $u \in W_{[n+1]}$ such that $u \nu_{[n+1]}=\nu_{[n+1]}$ and $w=x u$. Note that $u$ is equal to a product of the $r_{i}$ 's for $i \in[n+1]$ such that $\left\langle\nu_{[n+1]}, h_{i}\right\rangle=0$. Consequently, by using (3.1), we obtain

$$
S_{w}(\pi \otimes \eta)=S_{x u}(\pi \otimes \eta)=S_{x} S_{u}(\pi \otimes \eta)=S_{x}(\pi \otimes \eta) .
$$

This completes the proof of the proposition.

Let $n \in \mathbb{Z}_{\geq 0}$ be such that $n>p+(N+1) q$. Let $\mathbb{B}_{\max }^{[n]}$ denote the subset of $\mathbb{B}_{[n]}(\lambda) \otimes \mathbb{B}_{[n]}(\mu)$ consisting of all $[n]$-maximal elements, and set $\mathbb{B}_{\max , \nu}^{[n]}:=\mathbb{B}_{\max }^{[n]} \cap$ $\left(\mathbb{B}_{[n]}(\lambda) \otimes \mathbb{B}_{[n]}(\mu)\right)_{\nu}$ for $\nu \in P_{+}^{[n]}(\lambda, \mu)$. Then, by Lemma 4.7

$$
\mathbb{B}_{\max }^{[n]}=\bigsqcup_{\nu \in P_{+}^{[n]}(\lambda, \mu)} \mathbb{B}_{\max , \nu}^{[n]} .
$$

Also, we see by Lemmas 4.7 and 4.8 that

$$
\mathbb{B}_{\max }^{[n+1]}=\bigsqcup_{\xi \in P_{+}^{[n+1]}(\lambda, \mu)} \mathbb{B}_{\max , \xi}^{[n+1]}=\bigsqcup_{\nu \in P_{+}^{[n]}(\lambda, \mu)} \mathbb{B}_{\max , \nu_{[n+1]}^{[n+1]}} .
$$


Let $\nu \in P_{+}^{[n]}(\lambda, \mu)$, and let $x \in W_{[n+1]}$ be such that $x \nu_{[n+1]}=\nu$. By Proposition 4.9. we obtain an injective map $S_{x}$ from $\mathbb{B}_{\max , \nu_{[n+1]}}^{[n+1]}$ into $\mathbb{B}_{\max , \nu}^{[n]}$ :

$$
S_{x}: \mathbb{B}_{\max , \nu_{[n+1]}}^{[n+1]} \hookrightarrow \mathbb{B}_{\max , \nu}^{[n]}
$$

Proposition 4.10. Keep the setting above. The map $S_{x}: \mathbb{B}_{\max , \nu_{[n+1]}}^{[n+1]} \rightarrow \mathbb{B}_{\max , \nu}^{[n]}$ is bijective.

The proof of this proposition is given in $\$ 5$. In the rest of this subsection, we take and fix an arbitrary $m \in \mathbb{Z}_{\geq 0}$ such that $m>p+(N+1) q$.

Corollary 4.11. Every element $\pi \otimes \eta$ of $\mathbb{B}_{\max }^{[m]}$ is extremal.

Proof. It suffices to show that $\pi \otimes \eta$ is $[n]$-extremal for all $n \geq m$. We set $\nu:=$ $\operatorname{wt}(\pi \otimes \eta) \in P_{+}^{[m]}(\lambda, \mu)$. Since $\nu_{[m]}=\nu$ and $\nu_{[n+1]}=\left(\nu_{[n]}\right)_{[n+1]}$ for all $n \geq m$ by the definitions, we can easily show by induction on $n$, using Lemma 4.8, that $\nu_{[n]} \in P_{+}^{[n]}(\lambda, \mu)$ for all $n \geq m$. For each $n \geq m$, we take $x_{n} \in W_{[n+1]}$ such that $x_{n}\left(\nu_{[n+1]}\right)=x_{n}\left(\left(\nu_{[n]}\right)_{[n+1]}\right)=\nu_{[n]}$. Since $\nu_{[n]} \in P_{+}^{[n]}(\lambda, \mu)$ as seen above, Proposition 4.10 asserts that the map

$$
S_{x_{n}}: \mathbb{B}_{\max , \nu_{[n+1]}}^{[n+1]}=\mathbb{B}_{\max ,\left(\nu_{[n]}\right)_{[n+1]}}^{[n+1]} \rightarrow \mathbb{B}_{\max , \nu_{[n]}}^{[n]}
$$

is bijective.

Now, fix $n \in \mathbb{Z}_{\geq 0}$ such that $n \geq m$, and set $y_{n}:=x_{m} x_{m+1} \cdots x_{n-2} x_{n-1}$. Note that $y_{n} \in W_{[n]}$. Then, the argument above shows that the composite $S_{y_{n}}=$ $S_{x_{m}} S_{x_{m+1}} \cdots S_{x_{n-2}} S_{x_{n-1}}$ yields a bijective map from $\mathbb{B}_{\max , \nu_{[n]}}^{[n]}$ onto $\mathbb{B}_{\max , \nu}^{[m]}$ as follows:

$\mathbb{B}_{\max , \nu_{[n]}}^{[n]} \stackrel{S_{x_{n-1}}}{\longrightarrow} \mathbb{B}_{\max , \nu_{[n-1]}}^{[n-1]} \stackrel{S_{x_{n-2}}}{\longrightarrow} \cdots \stackrel{S_{x_{m+1}}}{\longrightarrow} \mathbb{B}_{\max , \nu_{[m+1]}}^{[m+1]} \stackrel{S_{x_{m}}}{\longrightarrow} \mathbb{B}_{\max , \nu_{[m]}}^{[m]}=\mathbb{B}_{\max , \nu}^{[m]}$.

Consequently, the element $S_{y_{n}^{-1}}(\pi \otimes \eta)$ is contained in $\mathbb{B}_{\max , \nu_{[n]}^{[n]}}$, and hence it is an $[n]$-maximal element. Because $\mathbb{B}(\lambda) \otimes \mathbb{B}(\mu)$ is a normal $U_{q}(\mathfrak{g})$-crystal, it follows that $S_{y_{n}^{-1}}(\pi \otimes \eta)$ is $[n]$-extremal, and hence so is that $\pi \otimes \eta$. This proves the corollary.

Proposition 4.12. Each connected component of $\mathbb{B}(\lambda) \otimes \mathbb{B}(\mu)$ contains a unique element of $\mathbb{B}_{\max }^{[m]}$.

Proof. Let $\pi \otimes \eta \in \mathbb{B}(\lambda) \otimes \mathbb{B}(\mu)$, and take $n \in \mathbb{Z}_{\geq 0}$, with $n \geq m$, such that $\pi \otimes \eta \in \mathbb{B}_{[n]}(\lambda) \otimes \mathbb{B}_{[n]}(\mu)$ (see (3.7)). From Remark 3.18, we see that each connected component of $\mathbb{B}_{[n]}(\lambda) \otimes \mathbb{B}_{[n]}(\mu)$ is isomorphic, as a $U_{q}\left(\mathfrak{g}_{[n]}\right)$-crystal, to the crystal basis of a finite-dimensional irreducible $U_{q}\left(\mathfrak{g}_{[n]}\right)$-module. Therefore, there exists a monomial $X$ in the Kashiwara operators $e_{i}$ for $i \in[n]$ such that $X(\pi \otimes \eta) \in \mathbb{B}_{\max }^{[n]}$. Let $\xi \in P$ be the weight of $X(\pi \otimes \eta)$. Note that $\xi \in P_{+}^{[n]}(\lambda, \mu)$ by Lemma 4.7 We set $\nu:=\Theta_{m}^{-1} \Theta_{m+1}^{-1} \cdots \Theta_{n-2}^{-1} \Theta_{n-1}^{-1}(\xi) \in P_{+}^{[m]}(\lambda, \mu)$. It is clear that $\xi=\nu_{[n]}$ by Lemma 4.8. Then, the argument in the proof of Corollary 4.11 shows that there exists $y \in W_{[n]}$ such that $S_{y^{-1}}$ yields a bijective map from $\mathbb{B}_{\max , \xi}^{[n]}=\mathbb{B}_{\max , \nu_{[n]}}^{[n]}$ onto $\mathbb{B}_{\max , \nu}^{[m]}$. In particular, we have $S_{y^{-1}} X(\pi \otimes \eta) \in \mathbb{B}_{\max , \nu}^{[m]} \subset \mathbb{B}_{\max }^{[m]}$. Also, observe that since $y \in W_{[n]}$, the element $S_{y^{-1}} X(\pi \otimes \eta)$ lies in the connected component of $\mathbb{B}(\lambda) \otimes \mathbb{B}(\mu)$ containing $\pi \otimes \eta$. Thus, we have proved that each connected component of $\mathbb{B}(\lambda) \otimes \mathbb{B}(\mu)$ contains an element of $\mathbb{B}_{\text {max }}^{[m]}$. 
It remains to prove the uniqueness assertion. Suppose that $b_{1}, b_{2} \in \mathbb{B}_{\max }^{[m]}$ are contained in the same connected component of $\mathbb{B}(\lambda) \otimes \mathbb{B}(\mu)$. We set $\nu:=\operatorname{wt}\left(b_{1}\right) \in$ $P_{+}^{[m]}(\lambda, \mu)$ and $\xi:=\operatorname{wt}\left(b_{2}\right) \in P_{+}^{[m]}(\lambda, \mu)$. Since $b_{1}$ and $b_{2}$ are both extremal by Corollary 4.11, we deduce from Theorem 3.20 and Corollary 3.21(1) that the connected component containing both $b_{1}$ and $b_{2}$ is isomorphic to $\mathbb{B}(\nu)$, and also to $\mathbb{B}(\xi)$. Consequently, by Corollary $3.21(3)$, we obtain $\nu \in W \xi$. If we take $n \in \mathbb{Z}_{\geq 0}$, with $n \geq m$, such that $\nu \in W_{[n]} \xi$, then we have $\nu_{[n]}=\xi_{[n]}$, which implies that $\nu=\xi$ by Lemma 4.8. Thus, $b_{1}$ and $b_{2}$ are both elements of weight $\nu$ in a connected component of $\mathbb{B}(\lambda) \otimes \mathbb{B}(\mu)$ isomorphic to $\mathbb{B}(\nu)$. Therefore, by Corollary 3.21(2), we conclude that $b_{1}=b_{2}$, as desired. This proves the proposition.

The following is the main result of this subsection (cf. [Kw2, Corollary 7.3] in type $A_{+\infty}$ and [Kw3, Proposition 5.13] in type $\left.A_{\infty}\right)$.

Theorem 4.13. Let $\lambda \in P_{+}$and $\mu \in E$. We take $m \in \mathbb{Z}_{\geq 0}$ such that $m>$ $p+(N+1) q$ as above. Then, we have the following decomposition into connected components:

$$
\mathbb{B}(\lambda) \otimes \mathbb{B}(\mu)=\bigoplus_{\nu \in P_{+}^{[m]}(\lambda, \mu)} \mathbb{B}(\nu)^{\oplus m_{\lambda, \mu}^{\nu},}
$$

where for each $\nu \in P_{+}^{[m]}(\lambda, \mu)$, the multiplicity $m_{\lambda, \mu}^{\nu}$ is equal to $\# \mathbb{B}_{\max , \nu}^{[m]}$.

Remark 4.14. If $\mathfrak{g}$ is of type $B_{\infty}$ (resp., $C_{\infty}, D_{\infty}$ ), then $\mathfrak{g}_{[m]}$ is a "reductive" Lie algebra of type $B_{m+1}$ (resp., $C_{m+1}, D_{m+1}$ ). Here we note that $m>p+(N+1) q \geq 2$. Furthermore, we know from Remark 3.18 that $\mathbb{B}_{[m]}(\lambda)$ (resp., $\mathbb{B}_{[m]}(\mu)$ ) is isomorphic, as a $U_{q}\left(\mathfrak{g}_{[m]}\right)$-crystal, to the crystal basis of the finite-dimensional irreducible $U_{q}\left(\mathfrak{g}_{[m]}\right)$-module $V_{[m]}\left(\lambda_{[m]}\right)=V_{[m]}(\lambda)$ (resp., $\left.V_{[m]}\left(\mu_{[m]}\right)\right)$ of highest weight $\lambda_{[m]}=\lambda$ (resp., $\left.\mu_{[m]}\right)$. Therefore, for each $\nu \in P_{+}^{[m]}(\lambda, \mu)$, the number $\# \mathbb{B}_{\max , \nu}^{[m]}$ is equal to the multiplicity $\left[V_{[m]}(\lambda) \otimes V_{[m]}\left(\mu_{[m]}\right): V_{[m]}(\nu)\right]$ of the finite-dimensional irreducible $U_{q}\left(\mathfrak{g}_{[m]}\right)$-module $V_{[m]}(\nu)$ of highest weight $\nu$ in the tensor product $U_{q}\left(\mathfrak{g}_{[m]}\right)$-module $V_{[m]}(\lambda) \otimes V_{[m]}\left(\mu_{[m]}\right)$. Thus,

$$
m_{\lambda, \mu}^{\nu}=\# \mathbb{B}_{\max , \nu}^{[m]}=\left[V_{[m]}(\lambda) \otimes V_{[m]}\left(\mu_{[m]}\right): V_{[m]}(\nu)\right]
$$

for each $\nu \in P_{+}^{[m]}(\lambda, \mu)$. In particular, the number of those elements $\nu \in P_{+}^{[m]}(\lambda, \mu)$ for which $m_{\lambda, \mu}^{\nu} \neq 0$ is finite, and hence the total number of connected components of $\mathbb{B}(\lambda) \otimes \mathbb{B}(\mu)$ is finite. See [NS, §5.2] for an explicit description of the number $\# \mathbb{B}_{\max , \nu}^{[m]}$ in terms of Littlewood-Richardson coefficients.

Proof of Theorem 4.13 . Let $\mathbb{B}$ be an arbitrary connected component of $\mathbb{B}(\lambda) \otimes \mathbb{B}(\mu)$. Then, we know from Proposition 4.12 that there exists a unique element $\pi \otimes \eta$ of $\mathbb{B}$ that is contained in $\mathbb{B}_{\text {max }}^{[m]}$; we set $\nu:=\operatorname{wt}(\pi \otimes \eta) \in P_{+}^{[m]}(\lambda, \mu)$. Since the element $\pi \otimes \eta \in \mathbb{B} \cap \mathbb{B}_{\max }^{[m]}$ is extremal by Corollary 4.11, we see from Theorem 3.20 and Corollary $3.21(1)$ that $\mathbb{B}$ is isomorphic, as a $U_{q}(\mathfrak{g})$-crystal, to $\mathbb{B}(\nu)$.

Proposition 4.12 and Corollary 4.11 together with Theorem 3.20 and Corollary $3.21(1)$, show that for each $\nu \in P_{+}^{[m]}(\lambda, \mu)$, there exists a one-to-one correspondence between the set of connected components of $\mathbb{B}(\lambda) \otimes \mathbb{B}(\mu)$ isomorphic to $\mathbb{B}(\nu)$ and the subset $\mathbb{B}_{\max , \nu}^{[m]}$ of $\mathbb{B}_{\max }^{[m]}$ consisting of all elements of weight $\nu$. This implies immediately that $m_{\lambda, \mu}^{\nu}=\# \mathbb{B}_{\max , \nu}^{[m]}$. Thus we have proved the theorem. 
4.4. The case $\lambda, \mu \in P_{+}$. Let $\lambda, \mu \in P_{+}$. In this case, we see from Theorem 3.20 and Remark 3.4 $(1)$ that $\mathbb{B}(\lambda)$ (resp., $\mathbb{B}(\mu)$ ) is isomorphic, as a $U_{q}(\mathfrak{g})$-crystal, to the crystal basis of the irreducible highest weight $U_{q}(\mathfrak{g})$-module of highest weight $\lambda$ (resp., $\mu$ ). For $\eta \in \mathbb{B}(\mu)$, we say that $\lambda+\eta$ is dominant (resp., [n]-dominant for $n \in \mathbb{Z}_{\geq 0}$ ) if $\left\langle\lambda+\eta(t), h_{i}\right\rangle \geq 0$ for all $t \in[0,1]_{\mathbb{R}}$ and $i \in I$ (resp., $i \in[n]$ ). Remark that such an element $\eta \in \mathbb{B}(\mu)$ is said to be " $\lambda$-dominant" (resp., " $\lambda$-dominant" with respect to $\left.\mathfrak{g}_{[n]}\right)$ in the terminology of [Li1. It is easy to verify that $\lambda+\eta$ is dominant (resp., [n]-dominant) if and only if $\pi_{\lambda} \otimes \eta \in \mathbb{B}(\lambda) \otimes \mathbb{B}(\mu)$ is maximal (resp., $[n]$-maximal).

Theorem 4.15. Let $\lambda, \mu \in P_{+}$. Then:

(1) We have the following decomposition into connected components:

$$
\mathbb{B}(\lambda) \otimes \mathbb{B}(\mu)=\bigoplus_{\substack{\eta \in \mathbb{B}(\mu) \\ \lambda+\eta \text { is dominant }}} \mathbb{B}(\lambda+w t(\eta)) .
$$

In particular, each connected component of $\mathbb{B}(\lambda) \otimes \mathbb{B}(\mu)$ is isomorphic, as a $U_{q}(\mathfrak{g})$ crystal, to $\mathbb{B}(\nu)$ for some $\nu \in P_{+}$.

(2) Let $\nu \in P_{+}$. If $L_{\nu} \neq L_{\lambda}+L_{\mu}$, then the multiplicity $m_{\lambda, \mu}^{\nu}$ of $\mathbb{B}(\nu)$ in the decomposition (4.12) is equal to 0 . If $L_{\nu}=L_{\lambda}+L_{\mu}$, then the multiplicity $m_{\lambda, \mu}^{\nu}$ of $\mathbb{B}(\nu)$ in the decomposition (4.12) is equal to the number

$$
\#\left\{\eta \in \mathbb{B}_{[n]}(\mu) \mid \lambda+\eta \text { is }[n] \text {-dominant, and } w t\left(\pi_{\lambda} \otimes \eta\right)=\nu\right\}
$$

for an arbitrary $n \in \mathbb{Z}_{\geq 3}$ such that $\lambda^{(j)}=L_{\lambda}, \mu^{(j)}=L_{\mu}$, and $\nu^{(j)}=L_{\nu}=L_{\lambda}+L_{\mu}$ for all $j \geq n$.

Proof. The formula (4.12) is just a restatement of the result in [Li2, §10]. Part (2) is essentially the same as [Le, Proposition 2.3.2] (see also its proof). Compare this with [Kw3, Proposition 4.10] in type $A_{\infty}$. Also, in [NS], another proof of part (2) is given by using LS paths.

Remark 4.16. If $\mathfrak{g}$ is of type $B_{\infty}$ (resp., $C_{\infty}, D_{\infty}$ ), then $\mathfrak{g}_{[n]}$ is a "reductive" Lie algebra of type $B_{n+1}$ (resp., $C_{n+1}, D_{n+1}$ ). Furthermore, we know from Remark 3.18 that $\mathbb{B}_{[n]}(\lambda)$ (resp., $\left.\mathbb{B}_{[n]}(\mu)\right)$ is isomorphic, as a $U_{q}\left(\mathfrak{g}_{[n]}\right)$-crystal, to the crystal basis of the finite-dimensional irreducible $U_{q}\left(\mathfrak{g}_{[n]}\right)$-module of highest weight $\lambda_{[n]}=\lambda$ (resp., $\mu_{[n]}=\mu$ ). Therefore, it follows from part (2) of Theorem 4.15, together with the result in [Li2, §10], that for each $\nu=\nu_{[n]} \in P_{+}$such that $L_{\nu}=L_{\lambda}+L_{\mu}$, the multiplicity $m_{\lambda, \mu}^{\nu}$ in the decomposition (4.12) is equal to the tensor product multiplicity of the corresponding finite-dimensional irreducible $U_{q}\left(\mathfrak{g}_{[n]}\right)$-modules. See [NS, §5.2] for an explicit description of this tensor product multiplicity in terms of Littlewood-Richardson coefficients. In particular, we have $m_{\lambda, \mu}^{\nu}<\infty$ for all $\nu \in P_{+}$. However, in the case $\lambda, \mu \in P_{+}$, the total number of connected components of $\mathbb{B}(\lambda) \otimes \mathbb{B}(\mu)$ is not finite in general. Compare this with the other cases, in which the total number of connected components of $\mathbb{B}(\lambda) \otimes \mathbb{B}(\mu)$ is finite (see Remarks 4.2 and 4.14 and Theorem 4.4).

4.5. The general case. Finally, in this subsection, we consider the decomposition (into connected components) of the tensor product $\mathbb{B}(\lambda) \otimes \mathbb{B}(\mu)$ for general $\lambda, \mu \in P$ such that $L_{\lambda}, L_{\mu} \geq 0$. Define $\lambda^{+}, \mu^{+} \in P_{+}$and $\lambda^{0}, \mu^{0} \in E$ as in Remark4.5. Then we have

$$
\mathbb{B}(\lambda) \otimes \mathbb{B}(\mu) \cong \mathbb{B}\left(\lambda^{0}\right) \otimes \mathbb{B}\left(\lambda^{+}\right) \otimes \mathbb{B}\left(\mu^{0}\right) \otimes \mathbb{B}\left(\mu^{+}\right)
$$


as $U_{q}(\mathfrak{g})$-crystals. Since $\lambda^{+} \in P_{+}$and $\mu^{0} \in E$, it follows from Theorem 4.13 that

$$
\mathbb{B}\left(\lambda^{0}\right) \otimes \mathbb{B}\left(\lambda^{+}\right) \otimes \mathbb{B}\left(\mu^{0}\right) \otimes \mathbb{B}\left(\mu^{+}\right) \cong \bigoplus_{\xi \in P_{+}^{[m]}\left(\lambda^{+}, \mu^{0}\right)}\left(\mathbb{B}\left(\lambda^{0}\right) \otimes \mathbb{B}(\xi) \otimes \mathbb{B}\left(\mu^{+}\right)\right)^{\oplus m_{\lambda^{+}, \mu_{0}}^{\xi}}
$$

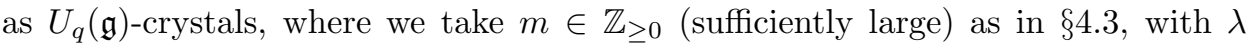
replaced by $\lambda^{+}$, and $\mu$ by $\mu^{0}$. If we define $\xi^{+} \in P_{+}$and $\xi^{0} \in E$ for each $\xi \in$ $P_{+}^{[m]}\left(\lambda^{+}, \mu^{0}\right)$ as in Remark 4.5, then we have

$$
\mathbb{B}\left(\lambda^{0}\right) \otimes \mathbb{B}(\xi) \otimes \mathbb{B}\left(\mu^{+}\right) \cong \mathbb{B}\left(\lambda^{0}\right) \otimes \mathbb{B}\left(\xi^{0}\right) \otimes \mathbb{B}\left(\xi^{+}\right) \otimes \mathbb{B}\left(\mu^{+}\right)
$$

as $U_{q}(\mathfrak{g})$-crystals. Since $\lambda^{0}, \nu^{0} \in E$, it follows from Theorem 4.1 that

$$
\mathbb{B}\left(\lambda^{0}\right) \otimes \mathbb{B}\left(\xi^{0}\right) \cong \bigoplus_{\zeta \in P_{-}^{[1, \infty)}} \mathbb{B}(\zeta)^{\oplus m_{\lambda_{+}^{0}, \xi_{\uparrow}^{0}}^{\zeta}} \quad \text { as } U_{q}(\mathfrak{g}) \text {-crystals. }
$$

Also, since $\xi^{+}, \mu^{+} \in P_{+}$, it follows from Theorem 4.15 that

$$
\mathbb{B}\left(\xi^{+}\right) \otimes \mathbb{B}\left(\mu^{+}\right) \cong \bigoplus_{\chi \in P_{+}} \mathbb{B}(\chi)^{\oplus m_{\xi^{+}, \mu^{+}}^{\chi}} \quad \text { as } U_{q}(\mathfrak{g}) \text {-crystals. }
$$

Combining these, we find that

$$
\mathbb{B}(\lambda) \otimes \mathbb{B}(\mu) \cong \bigoplus_{\substack{\xi \in P_{+}^{[m]}\left(\lambda^{+}, \mu^{0}\right) \\ \zeta \in P_{-}^{[1, \infty)}, \chi \in P_{+}}}(\mathbb{B}(\zeta) \otimes \mathbb{B}(\chi))^{\oplus m_{\lambda^{+}, \mu_{0}}^{\xi} m_{\lambda_{+}^{0}, \xi_{+}^{0}}^{m_{\xi^{+}, \mu^{+}}^{\chi}}}
$$

as $U_{q}(\mathfrak{g})$-crystals. Recall that $P_{-}^{[1, \infty)}$ is a complete set of representatives for $W$ orbits in $E$. Let $P / W$ denote the set of all $W$-orbits in $P$, which we regard as a subset of $P$ by taking a complete set of representatives for $W$-orbits in $P$ (recall Corollary $3.21(3)$ ). Now, using Theorem 4.4 and Remark 4.5, together with Proposition 4.6, we obtain from (4.15) the following theorem (cf. [Kw2, Corollary 7.4] in type $A_{+\infty}$ and [Kw3, Theorem 5.14] in type $A_{\infty}$ ).

Theorem 4.17. Let $\lambda, \mu \in P$ be integral weights of nonnegative levels. Namely, $L_{\lambda}, L_{\mu} \geq 0$. Then, we have the following decomposition into connected components:

$$
\mathbb{B}(\lambda) \otimes \mathbb{B}(\mu)=\bigoplus_{\nu \in P / W} \mathbb{B}(\nu)^{m_{\lambda, \mu}^{\nu}},
$$

where for each $\nu \in P / W$, the multiplicity $m_{\lambda, \mu}^{\nu}$ is given as follows:

$$
m_{\lambda, \mu}^{\nu}=\sum_{\xi \in P_{+}^{[m]}\left(\lambda^{+}, \mu^{0}\right)} m_{\lambda^{+}, \mu_{0}}^{\xi} m_{\lambda_{\dagger}^{0}, \xi_{\dagger}^{0}}^{\nu_{0}^{0}} m_{\xi^{+}, \mu^{+}}^{\nu^{+}}
$$

\section{Proof of Proposition 4.10}

In this section, we prove Proposition 4.10. For simplicity of notation, we give its proof only for the case of type $C_{\infty}$. The proofs for the other cases are similar. For details, see [NS, §5]. 
5.1. Basic notation for Young diagrams. Recall that $\mathcal{P}$ denotes the set of all partitions. We usually identify a partition $\rho \in \mathcal{P}$ with the corresponding Young diagram, which is also denoted by $\rho$ (see, for example, [F]). For $\rho, \kappa \in \mathcal{P}$, we write $\rho \subset \kappa$ if the Young diagram of $\rho$ is contained in the Young diagram of $\kappa$. In this case, we denote by $\kappa / \rho$ the skew Young diagram obtained from the Young diagram of $\kappa$ by removing that of $\rho$, and by $|\kappa / \rho|$ the total number of boxes in this skew Young diagram, i.e., $|\kappa / \rho|=|\kappa|-|\rho|$. It is well-known that for $\rho, \kappa, \omega \in \mathcal{P}$, the Littlewood-Richardson coefficient $\operatorname{LR}_{\rho, \kappa}^{\omega}$ is nonzero only if $\rho \subset \omega$ and $\kappa \subset \omega$. Also, the conjugate of the partition $\rho \in \mathcal{P}$ is denoted by ${ }^{t} \rho$. In what follows, for $L \in \mathbb{Z}_{\geq 0}$ and $\rho \in \mathcal{P}$, let $\iota_{L}(\rho)$ denote the partition whose Young diagram is obtained from the Young diagram of $\rho$ by inserting one row with exactly $L$ boxes between an appropriate pair of adjacent rows of the Young diagram of $\rho$ in such a way that the resulting diagram is also a Young diagram. By convention, we set $\iota_{L}(\rho)=\rho$ if $L=0$.

We fix $\ell \in \mathbb{Z}_{\geq 3}$. Since $\mathfrak{g}$ is assumed to be of type $C_{\infty}, \mathfrak{g}_{[\ell]}$ is a "reductive" Lie algebra of type $C_{\ell+1}$. Let $\rho=\left(\rho^{(0)} \geq \rho^{(1)} \geq \rho^{(2)} \geq \cdots\right) \in \mathcal{P}$ be a partition whose length $\ell(\rho)$ is less than or equal to $\ell+1$, and let $J$ be a finite subset of $\mathbb{Z}_{\geq 1}$. We define $\rho^{J, \ell} \in \mathcal{P}$ as follows (cf. [Ko2, $\left.\S 8\right]$ ). Write the conjugate partition ${ }^{t} \rho$ of $\rho$ as ${ }^{t} \rho=\left({ }^{t} \rho^{(0)} \geq{ }^{t} \rho^{(1)} \geq{ }^{t} \rho^{(2)} \geq \cdots\right) \in \mathcal{P}$. Note that the first part ${ }^{t} \rho^{(0)}$ is equal to the length $\ell(\rho)$ of $\rho$, which is less than or equal to $\ell+1$ by assumption. Then we set

$\mathbf{b}\left({ }^{t} \rho\right)=\left(b_{j}\right)_{j \in \mathbb{Z}_{\geq 1}}:=\left({ }^{t} \rho^{(0)}>{ }^{t} \rho^{(1)}-1>\cdots>{ }^{t} \rho^{(j-1)}-(j-1)>{ }^{t} \rho^{(j)}-j>\cdots\right)$ and define $\mathbf{b}\left({ }^{t} \rho\right)^{J, \ell}=\left(b_{j}^{J, \ell}\right)_{j \in \mathbb{Z}_{\geq 1}}$ as follows: for each $j \in \mathbb{Z}_{\geq 1}$,

$$
b_{j}^{J, \ell}= \begin{cases}b_{j} & \text { if } j \notin J, \\ (2 \ell+4)-b_{j} & \text { if } j \in J .\end{cases}
$$

Observe that $b_{j}^{J, \ell} \geq \ell+3$ for all $j \in J$, and that $b_{j}^{J, \ell} \neq b_{k}^{J, \ell}$ for all $j, k \in \mathbb{Z}_{\geq 1}$ such that $j \neq k$. Let $\tau$ be the (unique) finite permutation of the set $\mathbb{Z}_{\geq 1}$ such that

$$
b_{\tau(1)}^{J, \ell}>b_{\tau(2)}^{J, \ell}>\cdots>b_{\tau(j)}^{J, \ell}>b_{\tau(j+1)}^{J, \ell}>\cdots ;
$$

note that $\tau(j)=j$ for all $j>\max J$. Now we define $\rho^{J, \ell}$ to be the conjugate of the partition

$$
\left(b_{\tau(1)}^{J, \ell} \geq b_{\tau(2)}^{J, \ell}+1 \geq \cdots \geq b_{\tau(j)}^{J, \ell}+(j-1) \geq b_{\tau(j+1)}^{J, \ell}+j \geq \cdots\right) .
$$

Also, we define

$$
\operatorname{sgn}^{J, \ell}(\rho):=\operatorname{sgn}(\tau) \times(-1)^{\# J},
$$

where $\operatorname{sgn}(\tau)$ denotes the sign of the finite permutation $\tau$ of the set $\mathbb{Z}_{\geq 1}$.

5.2. Tensor product multiplicity formulas in terms of Littlewood-Richardson coefficients. In this subsection, following [Ko2, we review a tensor product multiplicity formula (in the case of type $C_{\infty}$ ) which we use in the proof of Proposition 4.10. Fix $\ell \in \mathbb{Z}_{\geq 3}$. For an $[\ell]$-dominant integral weight $\lambda \in P$, we denote by $\operatorname{ch} V_{[\ell]}(\lambda)$ the formal character of the finite-dimensional irreducible $U_{q}\left(\mathfrak{g}_{[\ell]}\right)$-module $V_{[\ell]}(\lambda)$ of highest weight $\lambda$. We set

$$
P_{+}^{[\ell]}:=\left\{\lambda \in P \mid \lambda \text { is }[\ell] \text {-dominant, and } \lambda^{(j)}=L_{\lambda} \text { for all } j \in \mathbb{Z}_{\geq \ell+1}\right\} .
$$


For each $\lambda \in P_{+}^{[\ell]}$, we define a sequence $\phi_{\ell}(\lambda)$ by

$$
\phi_{\ell}(\lambda)=\left(\lambda^{(\ell)}, \ldots, \lambda^{(1)},\left\langle\lambda^{(0)}\right\rangle, 0,0, \ldots\right) .
$$

Note that by Remark 2.2 , we have $\lambda^{(\ell)} \geq \cdots \geq \lambda^{(1)} \geq\left\langle\lambda^{(0)}\right\rangle \geq 0$. In addition, since $\mathfrak{g}$ is assumed to be of type $C_{\infty}$, we see that $\left\langle\lambda^{(0)}\right\rangle=\lambda^{(0)}$ and that $\phi_{\ell}(\lambda)$ is a partition.

For the rest of this subsection, we fix $\lambda, \mu \in P_{+}^{[\ell]}$. Because every weight of $V_{[\ell]}(\lambda)$ (resp., $\left.V_{[\ell]}(\mu)\right)$ is contained in the set $\lambda-\sum_{i \in[\ell]} \mathbb{Z}_{\geq 0} \alpha_{i}$ (resp., $\mu-\sum_{i \in[\ell]} \mathbb{Z}_{\geq 0} \alpha_{i}$ ), we deduce through use of (2.4) that every weight $\nu \in P$ of $V_{[\ell]}(\lambda) \otimes V_{[\ell]}(\mu)$ satisfies the condition that

$$
\nu^{(j)}=\lambda^{(j)}+\mu^{(j)}=L_{\lambda}+L_{\mu} \quad \text { for all } j \in \mathbb{Z}_{\geq \ell+1} .
$$

In particular, we have $L_{\nu}=L_{\lambda}+L_{\mu}$.

Theorem 5.1 ([Ko2, Theorem $6.6(2)]$ ). Under the notation and setting above, we have

$$
\operatorname{ch} V_{[\ell]}(\lambda) \times \operatorname{ch} V_{[\ell]}(\mu)=\sum_{\substack{\nu \in P_{+}^{[\ell]} \\ L_{\nu}=L_{\lambda}+L_{\mu}}} C_{\phi_{\ell}(\lambda), \phi_{\ell}(\mu)}^{\phi_{\ell}(\nu), \ell} \operatorname{ch} V_{[\ell]}(\nu),
$$

where for simplicity of notation, we set

$$
C_{\rho_{1}, \rho_{2}}^{\rho ; \ell}:=\sum_{\substack{J \subset \mathbb{Z}_{\geq 1}, \# J<\infty \\ \omega_{1}, \omega_{2}, \omega_{3} \in \mathcal{P}}} \operatorname{LR}_{\omega_{1}, \omega_{2}}^{\rho_{1}} \operatorname{LR}_{\omega_{2}, \omega_{3}}^{\rho_{2}} \operatorname{LR}_{\omega_{3}, \omega_{1}}^{\rho^{J, \ell}} \operatorname{sgn} n^{J, \ell}(\rho)
$$

for partitions $\rho_{1}, \rho_{2}, \rho \in \mathcal{P}$ whose lengths are less than or equal to $\ell+1$.

5.3. Proof of Proposition 4.10. The following proposition plays an essential role in the proof of Proposition 4.10.

Proposition 5.2. Let $L \in \mathbb{Z}_{\geq 0}$ and $n \in \mathbb{Z}_{\geq 3}$. Let $\rho_{1}, \rho_{2}, \rho \in \mathcal{P}$ be partitions satisfying the following conditions:

(i) The lengths of these partitions are all less than or equal to $n+1$.

(ii) The first part of $\rho_{1}$ is equal to $L$.

(iii) There hold the inequalities

$$
\begin{aligned}
& y_{1}:=\#\left\{1 \leq j \leq n+1 \mid \text { the } j \text {-th part of } \rho_{1} \text { is equal to } L\right\}>\left|\rho_{2}\right|, \\
& y:=\#\{1 \leq j \leq n+1 \mid \text { the } j \text {-th part of } \rho \text { is equal to } L\}>\left|\rho_{2}\right| .
\end{aligned}
$$

If we set $\kappa_{1}:=\iota_{L}\left(\rho_{1}\right)$ and $\kappa:=\iota_{L}(\rho)$, then we have $C_{\rho_{1}, \rho_{2}}^{\rho ; n}=C_{\kappa_{1}, \rho_{2}}^{\kappa ; n+1}$.

The proof of this proposition will be given in 5.4

Proof of Proposition 4.10. We fix $n \in \mathbb{Z}_{\geq 0}$ such that $n>p+(N+1) q$, and take $\nu \in P_{+}^{[n]}(\lambda, \mu)$ as in Proposition 4.10, Recall from Remark 3.18 that $\mathbb{B}_{[n]}(\lambda)$ (resp., $\left.\mathbb{B}_{[n]}(\mu)=\mathbb{B}_{[n]}\left(\mu_{[n]}\right)\right)$ is isomorphic, as a $U_{q}\left(\mathfrak{g}_{[n]}\right)$-crystal, to the crystal basis of the finite-dimensional irreducible $U_{q}\left(\mathfrak{g}_{[n]}\right)$-module of highest weight $\lambda$ (resp., $\left.\mu_{[n]}\right)$. Similar statements hold for $\mathbb{B}_{[n+1]}(\lambda)$ and $\mathbb{B}_{[n+1]}(\mu)=\mathbb{B}_{[n+1]}\left(\mu_{[n+1]}\right)$, respectively. Therefore, the sets $\mathbb{B}_{\max , \nu}^{[n]} \subset \mathbb{B}_{[n]}(\lambda) \otimes \mathbb{B}_{[n]}(\mu)$ and $\mathbb{B}_{\max , \nu_{[n+1]}}^{[n+1]} \subset \mathbb{B}_{[n+1]}(\lambda) \otimes$ $\mathbb{B}_{[n+1]}(\mu)$ are both finite sets. Because it is already shown that the map $S_{x}$ : $\mathbb{B}_{\max , \nu_{[n+1]}^{[n+1]}}^{[\ln } \rightarrow \mathbb{B}_{\max , \nu}^{[n]}$ is injective, it suffices to show that $\# \mathbb{B}_{\max , \nu_{[n+1]}}^{[n+1]}=\# \mathbb{B}_{\max , \nu}^{[n]}$. Also, recall from Remark 4.14 that the number $\# \mathbb{B}_{\max , \nu}^{[n]}$ (resp., $\# \mathbb{B}_{\max , \nu_{[n+1]}^{[n+1]}}$ ) is 
equal to the multiplicity of $V_{[n]}(\nu)$ (resp., $V_{[n+1]}\left(\nu_{[n+1]}\right)$ ) in the tensor product $V_{[n]}(\lambda) \otimes V_{[n]}\left(\mu_{[n]}\right)\left(\right.$ resp., $\left.V_{[n+1]}(\lambda) \otimes V_{[n+1]}\left(\mu_{[n+1]}\right)\right)$, and hence it is equal to the coefficient of $\operatorname{ch} V_{[n]}(\nu)\left(\right.$ resp., $\left.\operatorname{ch} V_{[n+1]}\left(\nu_{[n+1]}\right)\right)$ in the product $\operatorname{ch} V_{[n]}(\lambda) \times \operatorname{ch} V_{[n]}\left(\mu_{[n]}\right)$ (resp., $\left.\operatorname{ch} V_{[n+1]}(\lambda) \times \operatorname{ch} V_{[n+1]}\left(\mu_{[n+1]}\right)\right)$. Below we will use an explicit description of the numbers $\# \mathbb{B}_{\max , \nu}^{[n]}$ and $\# \mathbb{B}_{\max }^{[n+1]} \nu_{[n+1]}$ in terms of Littlewood-Richardson coefficients obtained from formula (5.4).

Since $n>p+(N+1) q>q$ and $\operatorname{Supp}(\mu) \subset[q-1]$, it follows from Lemma 2.3 that $\operatorname{Supp}\left(\mu_{[\ell]}\right) \subset[\ell]$ and $\mu_{[\ell]}^{(0)}=0$ for $\ell=n, n+1$, and hence that $\mu_{[\ell]} \in P_{+}^{[\ell]}$ for $\ell=n, n+1$. In addition, it is easily seen that $\phi_{n}\left(\mu_{[n]}\right)=\phi_{n+1}\left(\mu_{[n+1]}\right)=\mu_{\dagger}$ and that $|\mu|=\sum_{j \in \mathbb{Z}_{\geq 0}}\left|\mu^{(j)}\right|$ is equal to the sum $\left|\mu_{\dagger}\right|$ of all parts of $\mu_{\dagger}$. Here we note that $n-p+1 \geq(N+1) q+1>|\mu|=\left|\mu_{\dagger}\right|$. Also, we infer from Remark 2.1 and the choice of $n$ that

$$
\cdots=\lambda^{(n+1)}=\lambda^{(n)}=\cdots=\lambda^{(p)}=L_{\lambda}>\lambda^{(p-1)} \geq \cdots \geq \lambda^{(1)} \geq\left\langle\lambda^{(0)}\right\rangle=\lambda^{(0)} \geq 0,
$$

and hence that $\lambda$ is contained in both of the sets $P_{+}^{[n]}$ and $P_{+}^{[n+1]}$. Since $\nu \in$ $P_{+}^{[n]}(\lambda, \mu)\left(\subset P_{+}^{[n]}\right)$, we see from (4.7) that

$$
\left\{\begin{array}{l}
0 \leq\left\langle\nu^{(0)}\right\rangle \leq \nu^{(1)} \leq \cdots \leq \nu^{\left(u_{0}-1\right)}<L_{\lambda} \\
\nu^{\left(u_{0}\right)}=\nu^{\left(u_{0}+1\right)}=\cdots=\nu^{\left(u_{1}-1\right)}=L_{\lambda} \\
L_{\lambda}<\nu^{\left(u_{1}\right)} \leq \nu^{\left(u_{1}+1\right)} \leq \cdots \leq \nu^{(n)} \\
\nu^{(j)}=L_{\lambda} \text { for all } j \geq n+1
\end{array}\right.
$$

for some $0 \leq u_{0}<u_{1} \leq n+1$, with $u_{1}-u_{0}>n-p-N q \geq|\mu|=\left|\mu_{\dagger}\right|$. Furthermore, we deduce from Lemma 4.8 that $\nu_{[n+1]} \in P_{+}^{[n+1]}(\lambda, \mu)\left(\subset P_{+}^{[n+1]}\right)$ is given by

$$
\left\{\begin{array}{l}
\nu_{[n+1]}^{(j)}=\nu^{(j)} \quad \text { for } 0 \leq j \leq u_{0}-1, \\
\nu_{[n+1]}^{(j)}=\nu^{(j)}=L_{\lambda} \quad \text { for } u_{0} \leq j \leq u_{1}-1, \\
\nu_{[n+1]}^{\left(u_{1}\right)}=L_{\lambda}, \\
\nu_{[n+1]}^{(j)}=\nu^{(j-1)} \quad \text { for } u_{1}+1 \leq j \leq n+1, \\
\nu_{[n+1]}^{(j)}=\nu^{(j)}=L_{\lambda} \quad \text { for } j \geq n+2 .
\end{array}\right.
$$

Now, noting that $\phi_{n}(\lambda), \phi_{n+1}(\lambda)$ and $\phi_{n}(\nu), \phi_{n+1}\left(\nu_{[n+1]}\right)$ are all partitions since $\mathfrak{g}$ is assumed to be of type $C_{\infty}$, we infer from (5.4) that $\# \mathbb{B}_{\max , \nu}^{[n]}=C_{\phi_{n}(\lambda), \mu_{\dagger}}^{\phi_{n}(\nu) ; n}$ and $\# \mathbb{B}_{\max , \nu_{[n+1]}}^{[n+1]}=C_{\phi_{n+1}(\lambda), \mu_{\dagger}}^{\phi_{n+1}\left(\nu_{[n+1]}\right) ; n+1}$. Here we see from (5.6) that the first part of $\phi_{n}(\lambda)$ is equal to $L:=L_{\lambda}$ and that

$$
\begin{aligned}
& \#\left\{1 \leq j \leq n+1 \mid \text { the } j \text {-th part of } \phi_{n}(\lambda) \text { is equal to } L\right\}=n-p+1>\left|\mu_{\dagger}\right| \\
& \#\left\{1 \leq j \leq n+1 \mid \text { the } j \text {-th part of } \phi_{n}(\nu) \text { is equal to } L\right\}=u_{1}-u_{0}>\left|\mu_{\dagger}\right|
\end{aligned}
$$


Also, we deduce from (5.6), (5.7), (5.8) that $\phi_{n+1}(\lambda)=\iota_{L}\left(\phi_{n}(\lambda)\right)$ and $\phi_{n+1}\left(\nu_{[n+1]}\right)$ $=\iota_{L}\left(\phi_{n}(\nu)\right)$. Therefore, by Proposition 5.2, we conclude that

$$
\# \mathbb{B}_{\max , \nu}^{[n]}=C_{\phi_{n}(\lambda), \mu_{\dagger}}^{\phi_{n}(\nu) ; n}=C_{\phi_{n+1}(\lambda), \mu_{\dagger}}^{\phi_{n+1}\left(\nu_{[n+1]}\right) ; n+1}=\# \mathbb{B}_{\max , \nu_{[n+1]}}^{[n+1]},
$$

as desired.

5.4. Proof of Proposition [5.2. If $L=0$, then $\rho_{1}$ is the empty partition $\emptyset$ by assumption (ii), and hence so is $\kappa_{1}=\rho_{L}\left(\rho_{1}\right)=\rho_{1}$. In this case, we deduce from the definitions that

$$
C_{\emptyset, \rho_{2}}^{\rho ; n}=\left\{\begin{array}{ll}
1 & \text { if } \rho=\rho_{2}, \\
0 & \text { otherwise, }
\end{array} \quad C_{\emptyset, \rho_{2}}^{\kappa ; n+1}= \begin{cases}1 & \text { if } \kappa=\rho_{2}, \\
0 & \text { otherwise. }\end{cases}\right.
$$

Since $\kappa=\iota_{L}(\rho)=\rho$, we obtain $C_{\rho_{1}, \rho_{2}}^{\rho ; n}=C_{\emptyset, \rho_{2}}^{\rho ; n}=C_{\emptyset, \rho_{2}}^{\kappa ; n+1}=C_{\kappa_{1}, \rho_{2}}^{\kappa ; n}$, as desired.

Assume, therefore, that $L>0$. We set

$$
\begin{aligned}
& \mathcal{Q}\left(\rho_{2}\right):=\left\{\left(\omega_{2}, \omega_{3}\right) \in \mathcal{P} \times \mathcal{P} \mid \mathrm{LR}_{\omega_{2}, \omega_{3}}^{\rho_{2}} \neq 0\right\}, \\
& \mathcal{R}\left(\rho_{1}, \omega_{2}\right):=\left\{\omega_{1} \in \mathcal{P}\left|\omega_{1} \subset \rho_{1},\right| \rho_{1}|=| \omega_{1}|+| \omega_{2} \mid\right\} \quad \text { for } \omega_{2} \in \mathcal{P}, \\
& \mathcal{R}\left(\kappa_{1}, \omega_{2}\right):=\left\{\omega_{1} \in \mathcal{P}\left|\omega_{1} \subset \kappa_{1},\right| \kappa_{1}|=| \omega_{1}|+| \omega_{2} \mid\right\} \quad \text { for } \omega_{2} \in \mathcal{P} .
\end{aligned}
$$

We will show that:

(a) there hold

$$
\begin{aligned}
& C_{\rho_{1}, \rho_{2}}^{\rho ; n}= \sum_{\substack{J \subset[1, L] \\
\left(\omega_{2}, \omega_{3}\right) \in \mathcal{Q}\left(\rho_{2}\right) \\
\omega_{1} \in \mathcal{R}\left(\rho_{1}, \omega_{2}\right)}} \operatorname{LR}_{\omega_{1}, \omega_{2}}^{\rho_{1}} \operatorname{LR}_{\omega_{2}, \omega_{3}}^{\rho_{2}} \operatorname{LR}_{\omega_{3}, \omega_{1}}^{\rho^{J, n}} \operatorname{sgn}^{J, n}(\rho), \\
& C_{\kappa_{1}, \rho_{2}}^{\kappa ; n+1}=\sum_{\substack{J \subset[1, L] \\
\left(\omega_{2}, \omega_{3}\right) \in \mathcal{Q}\left(\rho_{2}\right) \\
\omega_{1} \in \mathcal{R}\left(\rho_{1}, \omega_{2}\right)}} \operatorname{LR}_{\iota_{L}\left(\omega_{1}\right), \omega_{2}}^{\iota_{L}\left(\rho_{1}\right)} \operatorname{LR}_{\omega_{2}, \omega_{3}}^{\rho_{2}} \operatorname{LR}_{\omega_{3}, \iota_{L}\left(\omega_{1}\right)}^{\iota_{L}\left(\rho^{J, n}\right)} \operatorname{sgn}^{J, n}(\rho) ;
\end{aligned}
$$

(b) for each $J \subset[1, L],\left(\omega_{2}, \omega_{3}\right) \in \mathcal{Q}\left(\rho_{2}\right)$, and $\omega_{1} \in \mathcal{R}\left(\rho_{1}, \omega_{2}\right)$, there hold

$$
\begin{aligned}
& \operatorname{LR}_{\iota_{L}\left(\omega_{1}\right), \omega_{2}}^{\iota_{L}\left(\rho_{1}\right)}=\operatorname{LR}_{\omega_{1}, \omega_{2}}^{\rho_{1}}, \\
& \operatorname{LR}_{\omega_{3}, \iota_{L}\left(\omega_{1}\right)}^{\iota_{L}\left(\rho^{J, n}\right)}=\operatorname{LR}_{\omega_{3}, \omega_{1}}^{\rho^{J, n}} .
\end{aligned}
$$

Then, substituting (5.11) and (5.12) into (5.10), we obtain

$$
\begin{aligned}
C_{\kappa_{1}, \rho_{2}}^{\kappa ; n+1}= & \sum_{\substack{J \subset[1, L] \\
\left(\omega_{2}, \omega_{3}\right) \in \mathcal{Q}\left(\rho_{2}\right) \\
\omega_{1} \in \mathcal{R}\left(\rho_{1}, \omega_{2}\right)}} \operatorname{LR}_{\iota_{L}\left(\omega_{1}\right), \omega_{2}}^{\iota_{L}\left(\rho_{1}\right)} \operatorname{LR}_{\omega_{2}, \omega_{3}}^{\rho_{2}} \operatorname{LR}_{\omega_{3}, \iota_{L}\left(\omega_{1}\right)}^{\iota_{L}\left(\rho^{J, n}\right)} \operatorname{sgn}^{J, n}(\rho) \\
= & \sum_{\substack{J \subset[1, L] \\
\left(\omega_{2}, \omega_{3}\right) \in \mathcal{Q}\left(\rho_{2}\right) \\
\omega_{1} \in \mathcal{R}\left(\rho_{1}, \omega_{2}\right)}} \operatorname{LR}_{\omega_{1}, \omega_{2}}^{\rho_{1}} \operatorname{LR}_{\omega_{2}, \omega_{3}}^{\rho_{2}} \operatorname{LR}_{\omega_{3}, \omega_{1}}^{\rho^{J, n}} \operatorname{sgn}^{J, n}(\rho) \\
= & C_{\rho_{1}, \rho_{2}}^{\rho ; n} \quad \text { by (5.9), }
\end{aligned}
$$

as desired. 
First, we show equations (5.9) and (5.10). The following claim can be easily verified by an elementary argument.

Claim 1. (1) Fix $\left(\omega_{2}, \omega_{3}\right) \in \mathcal{Q}\left(\rho_{2}\right)$ and $\omega_{1} \in \mathcal{R}\left(\rho_{1}, \omega_{2}\right)$. If $\operatorname{LR}_{\omega_{3}, \omega_{1}}^{\rho^{J, n}} \neq 0$ for a finite subset $J$ of $\mathbb{Z}_{\geq 1}$, then $J$ is contained in $[1, L]=\{1,2, \ldots, L\}$. Also, if $J \subset[1, L]$, then the number of parts of $\rho^{J, n}$ that are equal to $L$ is greater than or equal to $y$.

(2) Fix $\left(\omega_{2}, \omega_{3}\right) \in \mathcal{Q}\left(\rho_{2}\right)$ and $\omega_{1} \in \mathcal{R}\left(\kappa_{1}, \omega_{2}\right)$. If $\operatorname{LR}_{\omega_{3}, \omega_{1}}^{\kappa^{J, n+1}} \neq 0$ for a finite subset $J$ of $\mathbb{Z}_{\geq 1}$, then $J$ is contained in $[1, L]=\{1,2, \ldots, L\}$.

Also, from the definitions, we have

$$
C_{\rho_{1}, \rho_{2}}^{\rho ; n}=\sum_{\substack{J \subset \mathbb{Z}_{\geq 1}, \# J<\infty \\\left(\omega_{2}, \omega_{3}\right) \in \mathcal{Q}\left(\rho_{2}\right) \\ \omega_{1} \in \mathcal{R}\left(\kappa_{1}, \omega_{2}\right)}} \operatorname{LR}_{\omega_{1}, \omega_{2}}^{\rho_{1}} \operatorname{LR}_{\omega_{2}, \omega_{3}}^{\rho_{2}} \operatorname{LR}_{\omega_{3}, \omega_{1}}^{\rho^{J, n}} \operatorname{sgn}^{J, n}(\rho) .
$$

By using (5.13) and Claim 1(1), we obtain equation (5.9). Similarly, we deduce from the definitions and Claim 1)(2) that

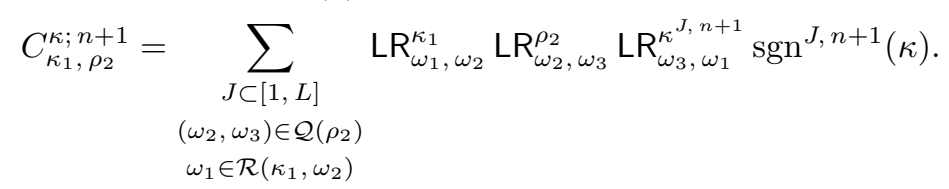

The following claims can also be shown by elementary arguments. Equation (5.10) follows immediately from (5.14) and these claims.

Claim 2. Fix $\left(\omega_{2}, \omega_{3}\right) \in \mathcal{Q}\left(\rho_{2}\right)$. For every $\omega_{1} \in \mathcal{R}\left(\rho_{1}, \omega_{2}\right)$, we have $\iota_{L}\left(\omega_{1}\right) \in$ $\mathcal{R}\left(\kappa_{1}, \omega_{2}\right)$. Thus, $\iota_{L}$ yields a map from $\mathcal{R}\left(\rho_{1}, \omega_{2}\right)$ to $\mathcal{R}\left(\kappa_{1}, \omega_{2}\right)$. Moreover, this map is bijective.

Claim 3. For each $J \subset[1, L]$, we have $\iota_{L}\left(\rho^{J, n}\right)=\kappa^{J, n+1}$ and $\operatorname{sgn}^{J, n}(\rho)=\operatorname{sgn}^{J, n+1}(\kappa)$.

Next, let us show equation (5.11). Since $\omega_{1} \subset \rho_{1}$ by the definition of $\mathcal{R}\left(\rho_{1}, \omega_{2}\right)$, it follows from assumption (ii) that the Young diagram of $\iota_{L}\left(\rho_{1}\right)$ (resp., $\iota_{L}\left(\omega_{1}\right)$ ) is obtained by adding one row having exactly $L$ boxes just above the top row of the Young diagram of $\rho_{1}$ (resp., $\omega_{1}$ ). This implies that the skew Young diagram $\iota_{L}\left(\rho_{1}\right) / \iota_{L}\left(\omega_{1}\right)$ is identical to the skew Young diagram $\rho_{1} / \omega_{1}$. Therefore, by the Littlewood-Richardson rule (see, for example, F, Chapter 5, Section 2, Proposition 3] or Ko2, §2]), we obtain (5.11).

Finally, let us show equation (5.12). Suppose that $\omega_{1} \not \subset \rho^{J, n}$. Let $j \in \mathbb{Z}_{\geq 1}$ be such that the $j$-th part of $\omega_{1}$ is greater than the $j$-th part of $\rho^{J, n}$. Since $\omega_{1} \subset \rho_{1}$ by the definition of $\mathcal{R}\left(\rho_{1}, \omega_{2}\right)$, it follows from assumption (ii) that

$$
L \geq \text { the } j \text {-th part of } \omega_{1}>\text { the } j \text {-th part of } \rho^{J, n},
$$

and hence that

$$
\begin{aligned}
\text { the }(j+1) \text {-st part of } \iota_{L}\left(\omega_{1}\right) & =\text { the } j \text {-th part of } \omega_{1} \\
>\text { the } j \text {-th part of } \rho^{J, n} & =\text { the }(j+1) \text {-st part of } \iota_{L}\left(\rho^{J, n}\right) .
\end{aligned}
$$

This implies that $\iota_{L}\left(\omega_{1}\right) \not \subset \iota_{L}\left(\rho^{J, n}\right)$. Thus, in this case, we obtain $\operatorname{LR}_{\omega_{3}, \iota_{L}\left(\omega_{1}\right)}^{J, n}=$ $0=\operatorname{LR}_{\omega_{3}, \omega_{1}}^{\rho^{J, n}}$. Also, if $\left|\rho^{J, n}\right| \neq\left|\omega_{1}\right|+\left|\omega_{3}\right|$, then it is obvious that $\left|\iota_{L}\left(\rho^{J, n}\right)\right| \neq$ $\left|\iota_{L}\left(\omega_{1}\right)\right|+\left|\omega_{3}\right|$ and hence that $\operatorname{LR}_{\omega_{3}, \iota_{L}\left(\omega_{1}\right)}^{\left.\iota^{J, n}\right)}=0=\mathrm{LR}_{\omega_{3}, \omega_{1}}^{\rho^{J, n}}$. We may, therefore, 
assume that $\omega_{1} \subset \rho^{J, n}$ and $\left|\rho^{J, n}\right|=\left|\omega_{1}\right|+\left|\omega_{3}\right|$. In this case, there exists $j_{0} \in \mathbb{Z}_{\geq 1}$ such that the $j_{0}$-th parts of $\rho^{J, n}$ and $\omega_{1}$ are both equal to $L$. Indeed, it follows from Claim 1(1) that the number of parts of $\rho^{J, n}$ that are equal to $L$ is greater than or equal to $y$. Let $j_{1}, j_{2} \in \mathbb{Z}_{\geq 1}$, with $j_{2}-j_{1} \geq y$, be such that the $j$-th part of $\rho^{J, n}$ is equal to $L$ for all $j_{1}+1 \leq j \leq j_{2}$. Suppose, contrary to our assertion, that the $j$-th part of $\omega_{1}$ is less than $L$ for all $j_{1}+1 \leq j \leq j_{2}$. Note that all parts of $\omega_{1} \in \mathcal{R}\left(\rho_{1}, \omega_{2}\right)$ are at most $L$. Then we must have $\left|\rho^{J, n} / \omega_{1}\right| \geq j_{2}-j_{1} \geq y>$ $\left|\rho_{2}\right|=\left|\omega_{2}\right|+\left|\omega_{3}\right| \geq\left|\omega_{3}\right|$, and hence $\left|\rho^{J, n}\right|>\left|\omega_{1}\right|+\left|\omega_{3}\right|$, which contradicts our assumption above. Consequently, the Young diagram of $\iota_{L}\left(\rho^{J, n}\right)\left(\right.$ resp., $\left.\iota_{L}\left(\omega_{1}\right)\right)$ is obtained by inserting one row having exactly $L$ boxes between the $j_{0}$-th row and the $\left(j_{0}+1\right)$-st row of the Young diagram of $\rho^{J, n}$ (resp., $\left.\omega_{1}\right)$. This implies that the skew Young diagram $\iota_{L}\left(\rho^{J, n}\right) / \iota_{L}\left(\omega_{1}\right)$ is identical to the skew Young diagram $\rho^{J, n} / \omega_{1}$. Therefore, by the Littlewood-Richardson rule, we obtain (5.12). This completes the proof of Proposition 5.2 .

\section{ACKNOWLEDGMEnTs}

We would like to express our sincere thanks to Jae-Hoon Kwon for several helpful comments concerning this work and also for his kindness.

\section{REFERENCES}

[AK] T. Akasaka and M. Kashiwara, Finite-dimensional representations of quantum affine algebras, Publ. Res. Inst. Math. Sci. 33 (1997), 839-867. MR.1607008 (99d:17017)

[F] W. Fulton, "Young tableaux: With applications to representation theory and geometry", London Mathematical Society Student Texts, Vol. 35, Cambridge University Press, Cambridge, 1997. MR:1464693 (99f:05119)

[HK] J. Hong and S.-J. Kang, "Introduction to Quantum Groups and Crystal Bases", Graduate Studies in Mathematics, Vol. 42, Amer. Math. Soc., Providence, RI, 2002. MR 1881971 (2002m:17012)

[J] A. Joseph, "Quantum Groups and Their Primitive Ideals", Ergebnisse der Mathematik und ihrer Grenzgebiete, Vol. 29, Springer-Verlag, Berlin, 1995. MR.1315966 (96d:17015)

[Kac] V. G. Kac, "Infinite Dimensional Lie Algebras", 3rd Edition, Cambridge University Press, Cambridge, UK, 1990. MR1104219 (92k:17038)

[Kas1] M. Kashiwara, On crystal bases of the $q$-analogue of universal enveloping algebras, Duke Math. J. 63 (1991), 465-516. MR.1115118 (93b:17045)

[Kas2] M. Kashiwara, The crystal base and Littelmann's refined Demazure character formula, Duke Math. J. 71 (1993), 839-858. MR1240605 (95b:17019)

[Kas3] M. Kashiwara, Crystal bases of modified quantized enveloping algebra, Duke Math. J. 73 (1994), 383-413. MR.1262212 (95c:17024)

[Kas4] M. Kashiwara, Similarity of crystal bases, in "Lie Algebras and Their Representations" (S.-J. Kang et al., Eds.), Contemp. Math., Vol. 194, pp. 177-186, Amer. Math. Soc., Providence, RI, 1996. MR 1395599 (97g:17013)

[Kas5] M. Kashiwara, On level-zero representations of quantized affine algebras, Duke Math. J. 112 (2002), 117-175. MR.1890649 (2002m:17013)

[Ko1] K. Koike, Representations of spinor groups and the difference characters of $S O(2 n), A d v$. Math. 128 (1997), 40-81. MR1451419 (98m:20052)

[Ko2] K. Koike, On representation of the classical groups, in "Selected Papers on Harmonic Analysis, Groups, and Invariants" (K. Nomizu, Ed.), Amer. Math. Soc. Transl. Ser. 2, Vol. 183, pp. 79-100, Amer. Math. Soc., Providence, RI, 1998. MR1615138

[Kw1] J.-H. Kwon, Demazure crystals of generalized Verma modules and a flagged RSK correspondence, J. Algebra 322 (2009), 2150-2179. MR2542836(2010i:17024)

[Kw2] J.-H. Kwon, Differential operators and crystals of extremal weight modules, Adv. Math. 222 (2009), 1339-1369. MR2554938 (2010h:17017)

[Kw3] J.-H. Kwon, Crystal duality and Littlewood-Richardson rule of extremal weight crystals, J. Algebra 336 (2011), 99-138. MR2802533 
[Kw4] J.-H. Kwon, Crystal bases of modified quantum groups and RSK correspondence, J. Combin. Theory Ser. A 118 (2011), 2131-2156. MR.2802192 (2012e:17038)

[Le] C. Lecouvey, Crystal bases and combinatorics of infinite rank quantum groups, Trans. Amer. Math. Soc. 361 (2009), 297-329. MR2439408 (2009k:17029)

[Li1] P. Littelmann, A Littlewood-Richardson rule for symmetrizable Kac-Moody algebras, Invent. Math. 116 (1994), 329-346. MR1253196 (95f:17023)

[Li2] P. Littelmann, Paths and root operators in representation theory, Ann. of Math. (2) 142 (1995), 499-525. MR1356780 (96m:17011)

[NS] S. Natio and D. Sagaki, Tensor product multiplicities for crystal bases of extremal weight modules over quantum infinite rank affine algebras of types $B_{\infty}, C_{\infty}$, and $D_{\infty}$, preprint 2010, arXiv:1003.2485.

Department of Mathematics, Tokyo Institute of Technology, 2-12-1 Oh-okayama, MEguro-Ku, TOKYO 152-8551, JAPAN

E-mail address: naito@math.titech.ac.jp

Institute of Mathematics, University of Tsukuba, Tsukuba, Ibaraki 305-8571, Japan

E-mail address: sagaki@math.tsukuba.ac.jp 